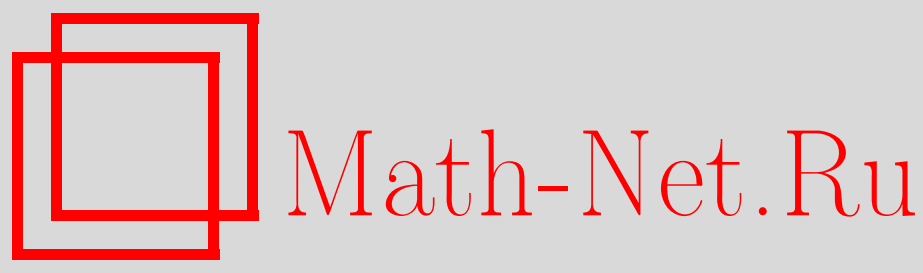

Ф. Ханизаде, А. В. Михайлов, Дж. П. Ванг, Преобразования Дарбу и рекурсионные операторы для дифференциально-разностных уравнений, ТМФ, 2013, том 177, номер $3,387-440$

DOI: https://doi.org/10.4213/tmf8550

Использование Общероссийского математического портала Math-Net.Ru подразумевает, что вы прочитали и согласны с пользовательским соглашением http://www.mathnet.ru/rus/agreement

Параметры загрузки:

IP : 54.224 .60 .19

26 апреля 2023 г., $12: 34: 43$

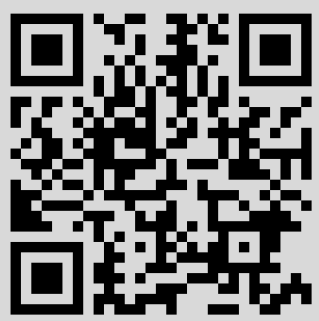




\title{
ФИЗИКА
}

Том 177, № 3

декабрь, 2013

(C) 2013 г. $\quad$ Ф. Ханизаде*, А. В. Михайлов ${ }^{\dagger}$, Дж. П. Ванг*

\section{ПРЕОБРАЗОВАНИЯ ДАРБУ И РЕКУРСИОННЫЕ ОПЕРАТОРЫ ДЛЯ ДИФФЕРЕНЦИАЛЬНО-РАЗНОСТНЫХ УРАВНЕНИЙ}

\begin{abstract}
Дан обзор двух концепций, непосредственно связанных с представлениями Лакса интегрируемых систем: преобразований Дарбу и рекурсионных операторов. Представлен обширный список интегрируемых дифференциально-разностных уравнений с их гамильтоновыми структурами, рекурсионными операторами, нетривиальными обобщенными симметриями и представлениями Дарбу-Лакса. K новым результатам относятся мультигамильтоновы структуры и рекурсионные операторы для интегрируемых уравнений типа Вольтерра, интегрируемых дискретизаций нелинейного уравнения Шредингера с производной, таких как цепочки Каупа-Ньюэлла, Чена-Ли-Лю и Абловица-Рамани-Сигура (Герджикова-Иванова). Также вычислены слабо нелокальные обратные операторы рекурсии.
\end{abstract}

Ключевые слова: симметрии, рекурсионный оператор, бигамильтонова структура, преобразование Дарбу, представление Лакса, интегрируемые уравнения.

DOI: $10.4231 / \operatorname{tmf} 8550$

\section{1. ВВЕДЕНИЕ}

Цель данной работы - дать описание мультигамильтоновых структур, рекурсионных операторов и представлений Дарбу-Лакса для широкого класса интегрируемых дифференциально-разностных уравнений. Некоторые из этих результатов хорошо известны, однако рассеяны в литературе. Во многих случаях мы дополняем картину, предъявляя явные выражения для гамильтоновых, симплектических, рекурсионных операторов и представлений Дарбу-Лакса. Представления Лакса нелинейных дифференциальных и разностных уравнений играют центральную роль в теории интегрируемых систем. Они позволяют применить метод обратной задачи для построения точных решений и изучения асимптотики задач с начальными

${ }^{*}$ School of Mathematics, Statistics and Actuarial Science, University of Kent, UK

${ }^{\dagger}$ Applied Mathematics Department, University of Leeds, UK.

E-mail: a.v.mikhailov@gmail.com 
условиями. Кроме того, они позволяют построить рекурсионный оператор, который генерирует бесконечные иерархии симметрий и законов сохранения. В настоящее время не существует общего метода нахождения представления Лакса для данного уравнения. Наиболее успешным из подходов является метод продолжения Уолквиста-Эстабрука [1]. Недавно эта задача была рассмотрена под другим углом [2]-[5]. Были изучены возможные редукции общего представления Лакса с использованием подхода, основанного на группах редукций [6]-[8] и далее ведущего к классификации представлений Лакса и соответствующих интегрируемых уравнений.

Понятие преобразований Дарбу происходит из классической дифференциальной геометрии [9]. Применение преобразований Дарбу к соответствующему представлению Лакса приводит к преобразованиям Беклунда и к построению новых решений интегрируемых систем. При этом сами преобразования Беклунда можно считать интегрируемой системой дифференциально-разностных уравнений. Такие дифференциально-разностные уравнения играют роль инфинитезимальных симметрий для интегрируемых уравнений в конечных разностях, которые получаются из условия Бьянки коммутативности преобразований Дарбу.

Основным объектом наших исследований являются системы дифференциальноразностных уравнений. Мы используем стандартные обозначения. Проиллюстрируем их на хорошо известном примере уравнения Вольтерра [10] (более подробное описание алгебраических свойств этого уравнения см. в п. 4.1), которое можно представить в виде

$$
u_{t}=u\left(u_{1}-u_{-1}\right)
$$

Здесь предполагается, что зависимая переменная $u$ является функцией $u(n, t)$ дискретной переменной $n \in \mathbb{Z}$ и непрерывной переменной $t$, а также введены обозначения

$$
u_{t}=\partial_{t} u(n, t), \quad u_{j}=u(n+j, t) .
$$

Далее мы будем опускать нижний индекс ноль, используя $u$ вместо $u_{0}$. Уравнение Вольтерра (1) заключает в себе бесконечную последовательность дифференциальных уравнений

$$
\partial_{t} u(n, t)=u(n, t)(u(n+1, t)-u(n-1, t)), \quad n \in \mathbb{Z} .
$$

Уравнение (1) обладает бесконечной иерархией симметрий, или, другими словами, оно совместно с бесконечной последовательностью эволюционных уравнений вида

$$
u_{t_{m}}=K_{m}\left(u_{m}, u_{m-1}, \ldots, u_{1-m}, u_{-m}\right), \quad m \in \mathbb{N},
$$

где $t_{1}=t, K_{1}=u\left(u_{1}-u_{-1}\right), K_{2}=u\left(u_{1} u_{2}+u_{1}^{2}+u u_{1}-u u_{-1}-u_{-1}^{2}-u_{-1} u_{-2}\right)$, а $K_{m}-$ некоторые полиномы переменных $u_{m}, u_{m-1}, \ldots, u_{1-m}, u_{-m}$. Совместность означает справедливость равенства $\partial_{t_{m}}\left(\partial_{t} u\right)=\partial_{t}\left(\partial_{t_{m}} u\right)$, т. е. обращение в нуль скобки Ли $\left[K_{1}, K_{m}\right]$, которая определяется по формуле

$$
\left[K_{1}, K_{m}\right]:=K_{m \star}\left(K_{1}\right)-K_{1 \star}\left(K_{m}\right),
$$


где $K_{m \star}$ означает производную Фреше

$$
K_{m \star}=\sum_{p=-m}^{m} \frac{\partial K_{m}}{\partial u_{p}} \mathcal{S}^{p},
$$

a $\mathcal{S}$ - оператор сдвига такой, что $\mathcal{S}^{j}\left(u_{k}\right)=u_{k+j}$, при этом для любой функции $f\left(u_{k_{1}}, \ldots, u_{k_{2}}\right)$ имеем $\mathcal{S}^{j}\left(f\left(u_{k_{1}}, \ldots, u_{k_{2}}\right)\right)=f\left(u_{k_{1}+j}, \ldots, u_{k_{2}+j}\right)$.

Симметрии уравнения Вольтерра можно построить с помощью рекурсионного оператора

$$
\mathcal{R}=u \mathcal{S}+u+u_{1}+u \mathcal{S}^{-1}+u\left(u_{1}-u_{-1}\right)(\mathcal{S}-1)^{-1} \frac{1}{u},
$$

а именно

$$
K_{m+1}=\mathcal{R}^{m}\left(K_{1}\right) .
$$

Векторные поля, соответствующие уравнению Вольтерра и его симметриям, являются разностными полиномами, т. е. элементами кольца разностных полиномов $R=[\mathbb{C}, u, \mathcal{S}]$, которое является кольцом без делителей нуля над полем комплексных чисел $\mathbb{C}$ с бесконечным числом переменных $u_{k}, k \in \mathbb{Z}$, и обладает автоморфизмом $\mathcal{S}$. Соответствующее поле частных $\mathcal{F}=(\mathbb{C}, u, \mathcal{S})$ является разностным полем рациональных функций переменных $u_{k}$ над полем $\mathbb{C}$, которое наследует автоморфизм $\mathcal{S}$. Разностные операторы представляют собой конечные суммы вида $\sum a_{k} \mathcal{S}^{k}$, где $a_{k} \in \mathcal{F}$ (определенная выше производная Фреше $K_{m \star}$ представляет собой пример разностного оператора). Разностные операторы естественным образом действуют на элементы $\mathcal{F}$. Как и в дифференциальном случае, элементы кольца $R$, поля $\mathcal{F}$ и разностные операторы называются соответственно локальными полиномами, локальными функциями и локальными операторами.

Рекурсионный оператор $\mathcal{R}$, заданный выражением (2), не является локальным. Он содержит локальную часть и член $u\left(u_{1}-u_{-1}\right)(\mathcal{S}-1)^{-1} u^{-1}$. Можно определить действие $\mathcal{R}$ на те элементы $\mathcal{F}$, которые принадлежат образу разностного оператора $u(\mathcal{S}-1)$. Легко проверить, что $K_{1}, K_{2} \in \operatorname{Im}(u(\mathcal{S}-1))$. Аналогично работе [11] можно показать, что все $K_{m+1}=\mathcal{R}^{m}\left(K_{1}\right), m \in \mathbb{N}$, являются разностными полиномами. Действие $\mathcal{R}$ на элементы $\operatorname{Im}(u(\mathcal{S}-1))$ определено неоднозначно. В самом деле, основное поле $\mathbb{C}$ является пространством ядра разностного оператора $u(\mathcal{S}-1)$ и, таким образом, для $a=u(\mathcal{S}-1)(b), b \in \mathcal{F}$, имеем $(\mathcal{S}-1)^{-1}\left(u^{-1} a\right)=b+\alpha$, где $\alpha \in \mathbb{C}$ - произвольная постоянная. Очевидно, действуя рекурсионным оператором $\mathcal{R}$, заданным формулой (2), на $K_{1}$, получим

$$
\mathcal{R}\left(u\left(u_{1}-u_{-1}\right)\right)=u\left(u_{1} u_{2}+u_{1}^{2}+u u_{1}-u u_{-1}-u_{-1}^{2}-u_{-1} u_{-2}\right)+\alpha u\left(u_{1}-u_{-1}\right) .
$$

Действие рекурсионного оператора $\mathcal{R}$ хорошо определено на последовательности линейных факторпространств

$$
\mathcal{K}_{1}=\operatorname{Span}_{\mathbb{C}}\left(K_{1}\right), \quad \mathcal{K}_{m}=\operatorname{Span}_{\mathbb{C}}\left(K_{1}, K_{2}, \ldots, K_{m}\right) / \operatorname{Span}_{\mathbb{C}}\left(K_{1}, K_{2}, \ldots, K_{m-1}\right),
$$

откуда следует, что при описании результата действия рекурсионного оператора на симметрию следует дать одного представителя соответствующего класса.

Рекурсионный оператор $\mathcal{R}$, заданный выражением (2), является псевдоразностным оператором. Его можно представить в виде $\mathcal{R}=B A^{-1}$, где $A$ и $B$ - разностные операторы. Например, можно взять $A=\mathcal{H}_{1}, B=\mathcal{H}_{2}$, где $\mathcal{H}_{1}$ и $\mathcal{H}_{2}$ - два гамильтоно- 
вых оператора для уравнения Вольтерра (см. п. 4.1). Заметим, что псевдоразностный оператор $\mathcal{R}$ является суммой локального (разностного) оператора и нелокального члена вида $P(\mathcal{S}-1)^{-1} Q$. Будем называть псевдоразностный оператор слабо нелокальным, если он представим в виде

$$
A+\sum_{i} P_{i}(\mathcal{S}-1)^{-1} Q_{i}
$$

где $A, P_{i}$ и $Q_{i}$ - разностные операторы, а сумма конечна (впервые подобная терминология была введена при изучении псевдодифференциальных гамильтоновых операторов в работе [12]). Таким образом, рекурсионный оператор $\mathcal{R}$, заданный выражением (2), является слабо нелокальным оператором. Более того, легко показать, что $\mathcal{R}^{m}, m \in \mathbb{N},-$ слабо нелокальный оператор. В большинстве случаев, изученных в настоящей работе, рекурсионные операторы являются слабо нелокальными. К исключениям относится рекурсионный оператор для цепочки Нариты-Ито-Богоявленского [13] (см. п. 4.5).

Интересно отметить, что в случае многокомпонентных систем интегрируемых разностных уравнений со слабо нелокальными рекурсионными операторами обратный оператор рекурсии зачастую также является слабо нелокальным. Это позволяет нам создать бесконечно много локальных симметрий, соответствующих обратным потокам. Например, ферромагнитная цепочка Гейзенберга [14] (подробное описание алгебраических свойств этого уравнения приводится в п. 4.17)

$$
\begin{aligned}
& u_{t}=(u-v)\left(u-u_{1}\right)\left(u_{1}-v\right)^{-1} \\
& v_{t}=(u-v)\left(v_{-1}-v\right)\left(u-v_{-1}\right)^{-1}
\end{aligned}
$$

обладает рекурсионным оператором

$$
\mathcal{R}=\left(\begin{array}{cc}
\frac{(u-v)^{2}}{\left(u_{1}-v\right)^{2}} \mathcal{S}-\frac{2\left(u-u_{1}\right)\left(v-v_{-1}\right)}{\left(u-v_{-1}\right)\left(u_{1}-v\right)} & -\frac{\left(u-u_{1}\right)^{2}}{\left(u_{1}-v\right)^{2}} \\
\frac{\left(v-v_{-1}\right)^{2}}{\left(u-v_{-1}\right)^{2}} & \frac{(u-v)^{2}}{\left(u-v_{-1}\right)^{2}} \mathcal{S}^{-1}
\end{array}\right)+2 K^{(1)}(\mathcal{S}-1)^{-1} Q^{(1)},
$$

где

$$
K^{(1)}=\left(\begin{array}{c}
\frac{(u-v)\left(u-u_{1}\right)}{u_{1}-v} \\
\frac{(u-v)\left(v_{-1}-v\right)}{u-v_{-1}}
\end{array}\right), \quad Q^{(1)}=\left(\frac{v-v_{-1}}{(u-v)\left(u-v_{-1}\right)}, \quad \frac{u-u_{1}}{(u-v)\left(u_{1}-v\right)}\right) .
$$

Оператор $\mathcal{R}$ является слабо нелокальным, причем его обратный оператор тоже слабо нелокальный:

$$
\mathcal{R}^{-1}=\left(\begin{array}{cc}
\frac{(u-v)^{2}}{\left(u_{-1}-v\right)^{2}} \mathcal{S}^{-1} & \frac{\left(u-u_{-1}\right)^{2}}{\left(u_{-1}-v\right)^{2}} \\
-\frac{\left(v-v_{1}\right)^{2}}{\left(u-v_{1}\right)^{2}} & \frac{(u-v)^{2}}{\left(u-v_{1}\right)^{2}} \mathcal{S}-\frac{2\left(u-u_{-1}\right)\left(v-v_{1}\right)}{\left(u-v_{1}\right)\left(u_{-1}-v\right)}
\end{array}\right)-2 K^{(-1)}(\mathcal{S}-1)^{-1} Q^{(-1)},
$$


где

$$
K^{(-1)}=\left(\begin{array}{c}
\frac{(u-v)\left(u_{-1}-u\right)}{u_{-1}-v} \\
\frac{(u-v)\left(v-v_{1}\right)}{u-v_{1}}
\end{array}\right), \quad Q^{(-1)}=\left(\frac{v-v_{1}}{(u-v)\left(u-v_{1}\right)}, \quad \frac{u-u_{-1}}{(u-v)\left(u_{-1}-v\right)}\right)
$$

Таким образом, ферромагнитная цепочка Гейзенберга имеет бесконечно много локальных симметрий $\mathcal{R}^{l}\left(K^{(1)}\right)$ и $\mathcal{R}^{-l}\left(K^{(-1)}\right)$ для всех $l \in \mathbb{N}$. Это явление обсуждалось в работе [15] для цепочек Абловица-Ладика и Бруски-Раниско. В настоящей работе мы вычислим слабо нелокальные обратные операторы рекурсии (если такие существуют) для всех многокомпонентных интегрируемых дифференциально-разностных уравнений. Проблема существования слабо нелокального обратного оператора для данного слабо нелокального разностного оператора все еще остается открытой.

Статья организована следующим образом. Сначала дан обзор двух тесно связанных проблем, относящихся к представлениям Лакса: преобразования Дарбу представления Лакса, из которого будут получены интегрируемые дифференциальноразностные уравнения, и вывод рекурсионного оператора для результирующих уравнений с использованием преобразования Дарбу. Мы поясним наши методы на двух типичных примерах: хорошо известного нелинейного уравнения Шредингера и деформации нелинейного уравнения Шредингера с производной, соответствующей диэдральной группе редукций $\mathbb{D}_{2}$.

Статья завершается длинным списком интегрируемых дифференциально-разностных уравнений, в котором будут перечислены сами уравнения, их гамильтоновы структуры, рекурсионные операторы, нетривиальные обобщенные симметрии и представления Лакса. Также будут включены частичные результаты, относящиеся к их мастер-симметриям. Для некоторых уравнений мы добавим дополнительные замечания относительно их связей с другими известными уравнениями и слабо нелокальные обратные операторы рекурсии, если такие имеются. Список далек от завершения. Аналогичный список $(1+1)$-мерных интегрируемых эволюционных дифференциальных уравнений в частных производных можно найти в работе [16].

В основном мы опираемся на те источники, в которых имеются полезные для нашей работы результаты об интегрируемых системах, хотя нами предприняты некоторые попытки и для выявления первоисточников. Составляя список, мы проверили и сопоставили материалы, собранные из обширной литературы. В наш список входит также ряд новых (насколько нам известно) результатов:

1) гамильтоновы операторы, симплектические операторы и рекурсионные операторы, приведенные в п. 4.4 для уравнений (68)-(70), а также связи между ними;

2) гамильтоновы операторы, симплектические операторы и рекурсионные операторы для цепочки Каупа-Ньюэлла (п. 4.14), цепочки Чен-Ли-Лю (п. 4.15) и цепочки Абловица-Рамани-Сигура (Герджикова-Иванова) (п. 4.16);

3) все слабо нелокальные обратные операторы рекурсии, если такие имеются (за исключением цепочек Абловица-Ладика и Бруски-Раниско, для которых нелокальные обратные операторы были известны). 


\section{2. ПРЕДСТАВЛЕНИЯ ЛАКСА И МАТРИЦЫ ДАРБУ}

С данной эволюционной нелинейной системой уравнений в частных производных

$$
\mathbf{u}_{t}=\mathbf{F}\left(\mathbf{u}, \mathbf{u}_{x}, \ldots, \mathbf{u}_{x, \ldots, x}\right), \quad \mathbf{u} \in \mathbb{C}^{m}
$$

интегрируемой методом обратной задачи [17]-[19], мы связываем пару линейных операторов

$$
L=D_{x}-U(\mathbf{u} ; \lambda), \quad A=D_{t}-V(\mathbf{u} ; \lambda),
$$

которую принято называть парой Лакса. Здесь $U, V$ - квадратные матрицы, элементы которых являются функциями зависимой переменной $\mathbf{u}$ и ее производных по $x$, а также некоторыми рациональными (в некоторых случаях эллиптическими) функциями спектрального параметра $\lambda$, такими, что уравнение (3) эквивалентно условию коммутирования этих операторов

$$
[L, A]=D_{t}(U)-D_{x}(V)+[U, V]=0 .
$$

Последнее уравнение часто называют представлением нулевой кривизны или представлением Лакса уравнения (3). В этой работе мы в основном рассматриваем $U$ и $V$ в виде $(2 \times 2)$-матриц, элементы которых рациональны по спектральному порядку $\lambda$.

Вообще говоря, симметрии эволюционного уравнения являются совместными с ним эволюционными уравнениями. Интегрируемое уравнение (3) имеет бесконечную последовательность коммутирующих симметрий

$$
\mathbf{u}_{t_{k}}=\mathbf{F}^{k}\left(\mathbf{u}, \mathbf{u}_{x}, \ldots, \mathbf{u}_{x, \ldots, x}\right), \quad k \in \mathbb{N},
$$

которые можно связать с коммутативной алгеброй линейных операторов

$$
A^{k}=D_{t_{k}}-V^{k}(\mathbf{u} ; \lambda), \quad\left[A^{i}, A^{j}\right]=0 .
$$

Как и уравнение (3), система (5) эквивалентна условию $\left[L, A^{k}\right]=0$. Оператор $A$ и уравнение (3) можно считать членами последовательности операторов $\left\{A^{k}\right\}$ и симметрий (5) соответственно при частных значениях $k$. Коммутирование операторов следует рассматривать как условия совместности бесконечной последовательности линейных задач

$$
D_{x}(\Psi)=U(\mathbf{u} ; \lambda) \Psi, \quad D_{t_{k}}(\Psi)=V^{k}(\mathbf{u} ; \lambda) \Psi
$$

т. е. условия существования общего фундаментального решения $\Psi$, $\operatorname{det} \Psi \neq 0$, всех этих задач,.

Посмотрим на преобразование Дарбу как на линейное отображение $\mathcal{S}$, действующее на фундаментальное решение

$$
\mathcal{S}: \Psi \mapsto \bar{\Psi}=M \Psi, \quad \operatorname{det} M \neq 0,
$$

так что матричная функция $\bar{\Psi}$ представляет собой фундаментальное решение линейных задач

$$
D_{x}(\bar{\Psi})=U(\overline{\mathbf{u}} ; \lambda) \bar{\Psi}, \quad D_{t_{k}}(\bar{\Psi})=V^{k}(\overline{\mathbf{u}} ; \lambda) \bar{\Psi},
$$


с новыми "потенциалами" $\overline{\mathbf{u}}$. Матрицу $M$ часто называют матрицей Дарбу. Элементы матрицы Дарбу $M$ являются рациональными (эллиптическими) функциями спектрального параметра $\lambda$. Определитель матрицы $M$ как функция $\lambda$ может обращаться в нуль только в конечном множестве точек на сфере Римана (параллелограмм периодов). Матрица Дарбу $M$ зависит от $\mathbf{u}, \overline{\mathbf{u}}$, а также может зависеть от некоторых вспомогательных функций $\mathbf{g}$ (или параметров, если $\mathbf{g}$ постоянны). (В качестве примеров таких функций g см. матрицы (18).) Итак, матрицу Дарбу обозначим $M=M(\mathbf{u}, \overline{\mathbf{u}}, \mathbf{g} ; \lambda)$. Из условия совместности (8) и (9) следует, что

$$
\begin{aligned}
D_{x}(M) & =U(\overline{\mathbf{u}} ; \lambda) M-M U(\mathbf{u} ; \lambda), \\
D_{t_{k}}(M) & =V^{k}(\overline{\mathbf{u}} ; \lambda) M-M V^{k}(\mathbf{u} ; \lambda) .
\end{aligned}
$$

Уравнения (10) и (11) - дифференциальные уравнения, связывающие два решения $\mathbf{u}$ и $\overline{\mathbf{u}}$ уравнений (3) и (5). В литературе их также часто называют преобразованиями Беклунда для уравнения (3).

Преобразование Дарбу отображает одну совместную систему (7) в другую (9). Оно задает отображение Дарбу $\mathcal{S}: \mathbf{u} \mapsto \overline{\mathbf{u}}$. Отображение $(8)$ обратимо $(\operatorname{det} M \neq 0)$ и его можно итерировать:

$$
\begin{aligned}
& \ldots \stackrel{\mathcal{S}}{\rightarrow} \Psi=M(\underline{\mathbf{u}}, \mathbf{u}, \underline{\mathbf{g}} ; \lambda) \underline{\Psi} \stackrel{\mathcal{S}}{\rightarrow} \bar{\Psi}=M(\mathbf{u}, \overline{\mathbf{u}}, \mathbf{g} ; \lambda) \Psi \stackrel{\mathcal{S}}{\rightarrow} \\
& \stackrel{\mathcal{S}}{\rightarrow} \overline{\bar{\Psi}}=M(\overline{\mathbf{u}}, \overline{\overline{\mathbf{u}}}, \overline{\mathbf{g}} ; \lambda) \bar{\Psi} \stackrel{\mathcal{S}}{\rightarrow} \overline{\bar{\Psi}}=M(\overline{\overline{\mathbf{u}}}, \overline{\overline{\mathbf{u}}}, \overline{\mathbf{g}} ; \lambda) \overline{\bar{\Psi}} \stackrel{\mathcal{S}}{\rightarrow} \cdots .
\end{aligned}
$$

Это отображение мотивирует введение обозначений

$$
\begin{aligned}
& \ldots, \quad \Psi_{-1}=\underline{\Psi}, \quad \Psi_{0}=\Psi, \quad \Psi_{1}=\bar{\Psi}, \quad \Psi_{2}=\overline{\bar{\Psi}}, \quad \ldots, \\
& \ldots, \quad \mathbf{u}_{-1}=\underline{\mathbf{u}}, \quad \mathbf{u}_{0}=\mathbf{u}, \quad \mathbf{u}_{1}=\overline{\mathbf{u}}, \quad \mathbf{u}_{2}=\overline{\overline{\mathbf{u}}}, \quad \ldots, \\
& \ldots, \quad \mathbf{g}_{-1}=\underline{\mathrm{g}}, \quad \mathrm{g}_{0}=\mathbf{g}, \quad \mathrm{g}_{1}=\overline{\mathrm{g}}, \quad \mathrm{g}_{2}=\overline{\overline{\mathbf{g}}}, \quad \ldots
\end{aligned}
$$

Свяжем с вершиной $k$ одномерной цепочки $\mathbb{Z}$ переменные $\Psi_{k}$ и $\mathbf{u}_{k} ;$ с ребром, соединяющим вершины $k$ и $k+1$, свяжем вспомогательные функции (параметры) $\mathbf{g}_{k}$ и матрицу $M_{k}=M\left(\mathbf{u}_{k}, \mathbf{u}_{k+1}, \mathbf{g}_{k} ; \lambda\right)$. В этих обозначениях отображение Дарбу $\mathcal{S}$ и $\mathcal{S}^{-1}$ увеличивает и уменьшает нижний индекс на единицу, поэтому мы назовем его $\mathcal{S}$-сдвигом или оператором сдвига $\mathcal{S}$. В дальнейшем мы часто будем опускать нижний индекс ноль и писать $\mathbf{u}, \mathbf{g}$ вместо $\mathbf{u}_{0}, \mathbf{g}_{0}$.

Уравнение (10) и последовательность (11) в этих обозначениях представляют собой иерархию совместных систем дифференциально-разностных уравнений. Если следующие из (10) и (11) уравнения являются эволюционными, то они образуют бесконечномерную алгебру Ли коммутирующих симметрий. Существование бесконечной алгебры коммутирующих симметрий часто берется за определение интегрируемости уравнения (и полной иерархии симметрий) [20]-[23].

Чтобы проиллюстрировать эти построения, рассмотрим два примера: хорошо известный пример нелинейного уравнения Шредингера и новые результаты, относящиеся к дифференциально-разностным уравнениям, соответствующим диэдральной группе редукций $\mathbb{D}_{2} \simeq \mathbb{Z}_{2} \times \mathbb{Z}_{2}$ (группа Клейна). Мы будем использовать эти примеры для пояснения вывода рекурсионных операторов. 
2.1. Нелинейное уравнение Шредингера. Нелинейное уравнение Шредингера

$$
\begin{aligned}
& 2 p_{t}=p_{x x}-8 p^{2} q, \\
& 2 q_{t}=-q_{x x}+8 q^{2} p
\end{aligned}
$$

имеет представление нулевой кривизны (4), где [24]

$$
\begin{aligned}
& U(\mathbf{u} ; \lambda)=\left(\begin{array}{cc}
0 & 2 p \\
2 q & 0
\end{array}\right)+\lambda\left(\begin{array}{cc}
1 & 0 \\
0 & -1
\end{array}\right), \quad \mathbf{u}=\left(\begin{array}{c}
p \\
q
\end{array}\right) \\
& V(\mathbf{u} ; \lambda)=\left(\begin{array}{cc}
-2 p q & p_{x} \\
-q_{x} & 2 p q
\end{array}\right)+\lambda\left(\begin{array}{cc}
0 & 2 p \\
2 q & 0
\end{array}\right)+\lambda^{2}\left(\begin{array}{cc}
1 & 0 \\
0 & -1
\end{array}\right) .
\end{aligned}
$$

Оно обладает бесконечной иерархией коммутирующих симметрий. Матричная часть соответствующих линейных операторов $A^{k}=D_{t_{k}}-V^{k}$ имеет вид

$$
V^{0}=J, \quad V^{k+1}=\lambda V^{k}+B^{k}(\mathbf{u}),
$$

где $J=\operatorname{diag}(1,-1)$, а $B^{k}(\mathbf{u})$ - матрицы с нулевым следом и с элементами, зависящими от $p, q$ и их производных по $x$. Матрицы $B^{k}(\mathbf{u})$ можно найти рекуррентным способом [25], в частности имеем

$$
\begin{gathered}
V^{1}=U(\mathbf{u} ; \lambda), \quad V^{2}=V(\mathbf{u} ; \lambda), \\
V^{3}=\lambda V(\mathbf{u} ; \lambda)+\frac{1}{2}\left(\begin{array}{cc}
2 p q_{x}-2 q p_{x} & p_{x x}-8 p^{2} q \\
q_{x x}-8 q^{2} p & 2 q p_{x}-2 p q_{x}
\end{array}\right) .
\end{gathered}
$$

Укажем симметрии, соответствующие $A^{0}, A^{1}, A^{2}$ и $A^{3}$ :

$$
\begin{array}{llrl}
p_{t_{0}} & =2 p, & p_{t_{1}} & =p_{x}, \\
q_{t_{0}} & =-2 q, & p_{t_{2}}=\frac{1}{2} p_{x x}-4 p^{2} q, & p_{t_{3}}=\frac{1}{4} p_{x x x}-6 p q p_{x} \\
q_{t_{1}}=q_{x}, & q_{t_{2}}=-\frac{1}{2} q_{x x}+4 q^{2} p, & q_{t_{3}}=\frac{1}{4} q_{x x x}-6 p q q_{x}
\end{array}
$$

Известно (см. [26], [27]), что любое преобразование Дарбу для нелинейного уравнения Шредингера является композицией следующих трех элементарных преобразований Дарбу с матрицами

$$
\begin{gathered}
M\left(\mathbf{u}, \mathbf{u}_{1}, f ; \lambda\right)=\left(\begin{array}{cc}
\lambda+f & p \\
q_{1} & 1
\end{array}\right), \quad N\left(\mathbf{u}, \mathbf{u}_{1}, h ; \lambda\right)=\left(\begin{array}{cc}
\lambda+h & p \\
p^{-1} & 0
\end{array}\right) \\
K\left(\mathbf{u}, \mathbf{u}_{1}, \alpha ; \lambda\right)=\left(\begin{array}{cc}
\alpha & 0 \\
0 & \alpha^{-1}
\end{array}\right)
\end{gathered}
$$

и обратных к ним.

Для каждой из указанных элементарных матриц Дарбу выведем отображение Дарбу и соответствующее дифференциально-разностное уравнение следующим образом. 
$(\boldsymbol{K}):$ Отображение, соответствующее матрице Дарбу $K$, не зависит от спектрального параметра $\lambda$ и переменных $\mathbf{u}, \mathbf{u}_{1}$. Более того, из уравнения (10) следует, что $\alpha-$ постоянная (не зависит от $x$ ), а $p_{1}=\alpha^{2} p, q_{1}=\alpha^{-2} q$. Таким образом, в этом случае отображение Дарбу является калибровочным преобразованием, соответствующим точечной симметрии уравнения (12) и ее иерархии симметрий.

$(\boldsymbol{N})$ : Подстановка $N\left(\mathbf{u}, \mathbf{u}_{1}, h ; \lambda\right)$ в уравнение $(10)$ дает

$$
q_{1}=\frac{1}{p}, \quad h_{x}=2 p_{1} q_{1}-2 p q, \quad p_{x}=-2 h p, \quad q_{1, x}=2 h q_{1},
$$

и, следовательно, мы получаем отображение Дарбу в следующем явном виде:

$$
p_{1}=p^{2} q-\frac{p}{4}\left(\frac{p_{x}}{p}\right)_{x}, \quad q_{1}=\frac{1}{p} .
$$

После замены переменных $p=e^{\phi}$ (и, как следствие, $q=e^{-\phi_{-1}}$ ) уравнения (19) преобразуются в следующую систему эволюционных уравнений:

$$
\phi_{x}=-2 h, \quad h_{x}=2 e^{\phi_{1}-\phi}-2 e^{\phi-\phi_{-1}},
$$

которая после исключения переменной $h$ принимает вид цепочки Тоды:

$$
\phi_{x x}=4 e^{\phi-\phi_{-1}}-4 e^{\phi_{1}-\phi} .
$$

Это бесконечная цепочка разностных уравнений для зависимых переменных $\phi_{n}$, $n \in \mathbb{Z}$.

Отметим, что $\phi=\ln p$ и $h=-\phi_{x} / 2$. Исключая производную по $x$ из (17) с помощью уравнений (19), получим симметрии цепочки Тоды (20):

$$
\begin{array}{rlrl}
3 \phi_{t_{0}}=2, & \phi_{t_{1}}=-2 h, & \phi_{t_{2}}=2 h^{2}-2(\mathcal{S}+1) e^{\phi-\phi_{-1}}, \\
h_{t_{0}}=0, & h_{t_{1}}=2(\mathcal{S}-1) e^{\phi-\phi_{-1}}, & h_{t_{2}}=-2(\mathcal{S}-1)\left(e^{\phi-\phi_{-1}}\left(h_{-1}+h\right)\right), \\
\phi_{t_{3}}=-2 h^{3}+2 e^{\phi-\phi_{-1}}\left(2 h+h_{-1}\right)+2 e^{\phi_{1}-\phi}\left(2 h+h_{1}\right), \\
h_{t_{3}}=2(\mathcal{S}-1)\left(\left(h_{-1}^{2}+h_{-1} h+h^{2}\right) e^{\phi-\phi_{-1}}+e^{2 \phi-2 \phi_{-1}}+(\mathcal{S}+1) e^{\phi-\phi_{-2}}\right) .
\end{array}
$$

Симметрии (21) имеют следующее представление Дарбу-Лакса:

$$
N_{t_{k}}-\mathcal{S}\left(U^{k}\right) N+N U^{k}=0, \quad N=\left(\begin{array}{cc}
\lambda+h & e^{\phi} \\
e^{-\phi} & 0
\end{array}\right),
$$

а матрицы $U^{0}, \ldots, U^{3}$ получаются из $V^{0}, \ldots, V^{3}$, введенных формулами (15) и (16), исключением производных по $x$ с помощью (19). Мы выведем рекурсионный оператор для производящих симметрий цепочки Тоды в разделе 3.

$(\boldsymbol{M})$ : Подстановка $M\left(\mathbf{u}, \mathbf{u}_{1}, f ; \lambda\right)$ в $(10)$ приводит к следующей системе дифференциально-разностных уравнений:

$$
f_{x}=2 p_{1} q_{1}-2 p q, \quad p_{x}=2 p_{1}-2 f p, \quad q_{x}=-2 q_{-1}+2 f_{-1} q .
$$


Симметрии этой системы следуют из представлений Лакса-Дарбу (11), в которых операторы $V^{k}$ следует взять в виде (15) и (16), исключив производные по $x$ с помощью системы (23):

$$
\begin{aligned}
& p_{t_{0}}=2 p, \quad p_{t_{1}}=2 p_{1}-2 f p, \quad p_{t_{2}}=2\left(f^{2} p-f p_{1}-f_{1} p_{1}-p^{2} q-p p_{1} q_{1}+p_{2}\right), \\
& q_{t_{0}}=-2 q, \quad q_{t_{1}}=-2 q_{-1}+2 f_{-1} q, \quad q_{1, t_{2}}=2\left(f_{-1} q+f q-f^{2} q_{1}+p q q_{1}+p_{1} q_{1}^{2}-q_{-1}\right), \\
& f_{t_{0}}=0, \quad f_{t_{1}}=2 p_{1} q_{1}-2 p q, \quad f_{t_{2}}=2(\mathcal{S}-1)\left(p q_{-1}+p_{1} q-\left(f_{-1}+f\right) p q\right) \text {. }
\end{aligned}
$$

Система (23) и ее симметрии (24) имеют первый интеграл $\Phi=f-p q_{1}$, т. е. $\Phi_{t_{k}}=0$. В самом деле, определитель $\operatorname{det} M\left(\mathbf{u}, \mathbf{u}_{1}, f ; \lambda\right)=\lambda+f-p q_{1}$ должен быть постоянным (не зависящим от $x, t_{k}$ ), поскольку матрицы $U, V^{k}$ бесследовые (теорема Абеля). Таким образом, можно положить $f_{k}=p_{k} q_{k+1}+\alpha_{k}$, где $\alpha_{k} \in \mathbb{C}-$ постоянная. Исключая $f$ из системы $(23)$, получим систему

$$
p_{x}=2 p_{1}-2 p^{2} q_{1}-2 \alpha p, \quad q_{x}=-2 q_{-1}+2 p_{-1} q^{2}+2 \alpha_{-1} q,
$$

симметрии которой получим из (24) тем же самым исключением $f$. Если $\alpha_{k}=0$, уравнения (25) становятся цепочкой Мерола-Раниско-Ту, указанной в п. 4.10, к которой применено обратимое преобразование $x=t / 2, p=u$ и $q_{1}=v$.

\section{2. Уравнения, которые соответствуют диэдральной группе редукций} $\mathbb{D}_{2} \simeq \mathbb{Z}_{2} \times \mathbb{Z}_{2}$. Интегрируемость системы

$$
\begin{aligned}
& 2 p_{t}=p_{x x}+4 q_{x}-8\left(p^{2} q\right)_{x}, \\
& 2 q_{t}=-q_{x x}+4 p_{x}-8\left(q^{2} p\right)_{x}
\end{aligned}
$$

была установлена в работе [21]. Это уравнение можно рассматривать как нетривиальную неоднородную деформацию нелинейного уравнения Шредингера с производной. Матричная часть соответствующей пары Лакса

$$
L(\mathbf{u} ; \lambda)=D_{x}-V^{1}(\mathbf{u} ; \lambda), \quad A(\mathbf{u} ; \lambda)=D_{t}-V^{2}(\mathbf{u} ; \lambda)
$$

имеет вид

$$
\begin{aligned}
& V^{1}(\mathbf{u} ; \lambda)=2 p \mathbf{a}_{1}(\lambda)+2 q \mathbf{a}_{2}(\lambda)+2 \mathbf{a}_{3}(\lambda), \\
& V^{2}(\mathbf{u} ; \lambda)=w(\lambda) V^{1}(\mathbf{u} ; \lambda)+\frac{p_{x}-4 p^{2} q}{2} \mathbf{a}_{1}(\lambda)-\frac{q_{x}+4 q^{2} p}{2} \mathbf{a}_{2}(\lambda)-2 p q \mathbf{a}_{3}(\lambda),
\end{aligned}
$$

где

$$
\mathbf{a}_{1}(\lambda)=\left(\begin{array}{cc}
0 & \lambda \\
\lambda^{-1} & 0
\end{array}\right), \quad \mathbf{a}_{2}(\lambda)=\left(\begin{array}{cc}
0 & \lambda^{-1} \\
\lambda & 0
\end{array}\right), \quad \mathbf{a}_{3}(\lambda)=\frac{\lambda^{2}-\lambda^{-2}}{2}\left(\begin{array}{cc}
1 & 0 \\
0 & -1
\end{array}\right) .
$$

Матрицы $V^{k}(\mathbf{u} ; \lambda)$ инвариантны по отношению к группе (группе редукций [8]), порожденной преобразованиями

$$
\begin{aligned}
& V^{k}(\mathbf{u} ; \lambda) \mapsto\left(\begin{array}{cc}
1 & 0 \\
0 & -1
\end{array}\right) V^{k}(\mathbf{u} ;-\lambda)\left(\begin{array}{cc}
1 & 0 \\
0 & -1
\end{array}\right), \\
& V^{k}(\mathbf{u} ; \lambda) \mapsto\left(\begin{array}{cc}
0 & 1 \\
1 & 0
\end{array}\right) V^{k}\left(\mathbf{u} ; \lambda^{-1}\right)\left(\begin{array}{cc}
0 & 1 \\
1 & 0
\end{array}\right),
\end{aligned}
$$


которая изоморфна диэдральной группе $\mathbb{D}_{2} \simeq \mathbb{Z}_{2} \times \mathbb{Z}_{2}$ (группе Клейна). Функция

$$
w(\lambda)=\frac{\lambda^{2}+\lambda^{-2}}{2}
$$

является элементарной автоморфной функцией, соответствующей группе Мёбиуса $\left(w(\lambda)=w(-\lambda)=w\left(\lambda^{-1}\right)\right)$, при этом любая рациональная автоморфная функция этой группы является рациональной функцией $w(\lambda)$ [3]. Иерархия высших симметрий системы (26) порождается операторами Лакса $A^{k}=D_{t_{k}}-V^{k}$, где матрицы $V^{k}$ имеют вид

$$
V^{k+1}=w(\lambda) V^{k}+r_{1}^{k} \mathbf{a}_{1}(\lambda)+r_{2}^{k} \mathbf{a}_{2}(\lambda)+r_{3}^{k} \mathbf{a}_{3}(\lambda),
$$

а коэффициенты $r_{1}^{k}, r_{2}^{k}, r_{3}^{k}$ являются полиномами по $p, q$ и их производных по $x$, причем их можно построить рекуррентным образом.

В работе [28] показано, что элементарную матрицу Дарбу оператора Лакса (27) можно представить в виде

$$
M\left(p, q_{1}, f, g ; \lambda\right)=f\left(w(\lambda) \mathbf{I}+\mathbf{a}_{3}(\lambda)+p \mathbf{a}_{1}(\lambda)+q_{1} \mathbf{a}_{2}(\lambda)+g \mathbf{I}\right),
$$

где $\mathbf{I}$ - единичная матрица. Все матрицы $V^{k}$ бесследовые, поэтому определитель

$$
\operatorname{det} M\left(p, q_{1}, f, g ; \lambda\right)=f^{2}\left(2 w(\lambda)\left(g-p q_{1}\right)+1+g^{2}-p^{2}-q_{1}^{2}\right)=2 w(\lambda) \Phi^{1}+\Phi^{2}
$$

не зависит от $x$ и $t_{k}$. Таким образом, у нас есть два инварианта (два первых интеграла)

$$
\Phi^{1}=f^{2}\left(g-p q_{1}\right), \quad \Phi^{2}=f^{2}\left(1+g^{2}-p^{2}-q_{1}^{2}\right) .
$$

Выбором подходящего масштаба $M \rightarrow \gamma M$ можно получить $\Phi^{1}=1\left(\right.$ если $\left.\Phi^{1} \neq 0\right)$ или $\Phi^{2}=1$ (если $\left.\Phi^{2} \neq 0\right)$.

Существуют три существенно различных случая [28]:

1) $\Phi^{1}=0, \Phi^{2}=1$, определитель является постоянным (не зависящим от $\lambda$ );

2) $\Phi^{1}=1, \Phi^{2}= \pm 2$, определитель имеет два двойных нуля и является квадратом рациональной функции от $\lambda$;

3) $\Phi^{1}=1, \Phi^{2}=2 \alpha$, определитель имеет четыре различных нуля на комплексной плоскости $\lambda$ (предполагается, что $\alpha \neq \pm 1$ ).

В случае 1 имеем

$$
g=p q_{1}
$$

и $f$ находится как решения уравнения $f^{2}\left(1-p^{2}\right)\left(1-q_{1}^{2}\right)=1$. Таким образом, определяется матрица $M\left(p, q_{1}, f, p q_{1} ; \lambda\right)$ в формуле (32). Подставляя в уравнение (10) эту матрицу, а также $V^{1}(\mathbf{u} ; \lambda)$ из уравнения $(27)$ вместо $U(\mathbf{u} ; \lambda)$, получим систему

$$
p_{x}=2\left(1-p^{2}\right)\left(q_{1}-q\right), \quad q_{x}=2\left(1-q^{2}\right)\left(p-p_{-1}\right),
$$

которую можно представить в виде скалярного уравнения для одной функции $v_{2 n}=$ $p_{n}, v_{2 n-1}=q_{n}$

$$
v_{x}=\left(1-v^{2}\right)\left(v_{1}-v_{-1}\right) .
$$


В случае 2 положим $2 \Phi^{1}+\Phi^{2}=0$ (другой выбор знака в конечном итоге привел бы к точечно-эквивалентной системе). Имеем

$$
2 \Phi^{1}+\Phi^{2}=f^{2}\left(1+g+p+q_{1}\right)\left(1+g-p-q_{1}\right)=0
$$

Выберем $1+g+p+q_{1}=0$ (выбор $1+g-p-q_{1}=0$ привел бы к точечно-эквивалентной системе). Тогда подстановка в уравнение $(10)$ матрицы $M\left(p, q_{1}, f,-p-q_{1}-1 ; \lambda\right)$, а также $V^{1}(\mathbf{u} ; \lambda)$ из уравнения $(27)$ вместо $U(\mathbf{u} ; \lambda)$ приводит к системе

$$
p_{x}=2(1+p)(\mathcal{S}-1)(q-p-p q), \quad q_{1, x}=2\left(1+q_{1}\right)(\mathcal{S}-1)(p-q-p q) .
$$

В общем случае 3 элементарного преобразования Дарбу имеем

$$
\Phi^{2}-2 \alpha \Phi^{1}=\left(g^{2}-2 \alpha g-p^{2}-q_{1}^{2}+2 \alpha p q_{1}+1\right) f^{2}=0, \quad f=\frac{1}{\sqrt{g-p q_{1}}} .
$$

Подстановка в уравнение $(10)$ матрицы $M\left(p, q_{1}, f, g ; \lambda\right)$, а также $V^{1}(\mathbf{u} ; \lambda)$ из уравнения $(27)$ вместо $U(\mathbf{u} ; \lambda)$ приводит к системе

$$
\begin{aligned}
p_{x} & =2 g\left(p_{1}-p\right)+2 q_{1}-2 q-2 p\left(p_{1} q_{1}-p q\right), \\
q_{1, x} & =2 g\left(q_{1}-q\right)+2 p_{1}-2 p-2 q_{1}\left(p_{1} q_{1}-p q\right), \\
g_{x} & =2 p\left(p_{1}-p\right)+2 q_{1}\left(q_{1}-q\right)-2 g\left(p_{1} q_{1}-p q\right), \\
f_{x} & =2 f\left(p_{1} q_{1}-p q\right) .
\end{aligned}
$$

Инварианты $\Phi^{1}, \Phi^{2}$ являются первыми интегралами этой системы. Таким образом, функции $f$ и $g$ можно исключить с помощью первых интегралов (38). Отметим, что параметр $\alpha$ в уравнении (38) не зависит от $x, t_{k}$, но он может зависеть от переменной сдвига, так что $\mathcal{S}(\alpha)=\alpha_{1}$.

\section{3. РЕКУРСИОННЫЙ ОПЕРАТОР ДЛЯ ДИФФЕРЕНЦИАЛЬНО-РАЗНОСТНЫХ УРАВНЕНИЙ}

В этом разделе мы выведем рекурсионный оператор с помощью представления Дарбу-Лакса дифференциально-разностного уравнения. Наше построение является естественным обобщением метода, используемого в теории интегрируемых уравнений в частных производных [25], [29]-[32]. Основная идея метода заключается в том, что матрицы $V^{k}(\mathbf{u}, \lambda)$ операторов $A^{k}=D_{t_{k}}-V^{k}(\mathbf{u}, \lambda)$, соответствующих иерархии, связаны соотношением

$$
V^{k+1}(\mathbf{u}, \lambda)=\mu(\lambda) V^{k}(\mathbf{u}, \lambda)+B^{k}(\mathbf{u}, \lambda)
$$

где $\mu(\lambda)$ является рациональным (эллиптическим в случае уравнения Ландау-Лифшица) множителем, а $B^{k}(\mathbf{u}, \lambda)$ - рациональная матрица с фиксированным (т. е. не зависящим от $k$ ) девизором полюсов. Если система вместе со своей парой Лакса получена в результате редукции из группы $G$, тогда множитель $\mu(\lambda)$ является элементарной автоморфной функцией [3] конечной группы редукций, или, в эллиптическом случае, одним из генераторов $G$-инвариантного подкольца координатного 
кольца [30]. Матрица $B^{k}(\mathbf{u}, \lambda)$ зависит также и от зависимых переменных и и их производных по $x$.

Подстановка выражения (39) в представление Лакса $\left[L, A^{k}\right]=0$ (6) приводит к уравнению

$$
D_{t_{k+1}}(L)=\mu(\lambda) D_{t_{k}}(L)-B^{k} L+L B^{k} .
$$

Выразим матрицу $B^{k}$ через переменные $\mathbf{u}, \mathbf{u}_{t_{k}}, \mathbf{u}_{t_{k+1}}$ и производные по $x$ от $\mathbf{u}$ с помощью уравнения (40). Тогда уравнение (40) можно рассматривать как рекуррентное соотношение $\mathbf{u}_{t_{k+1}}=\mathcal{R}\left(\mathbf{u}_{t_{k}}\right)$, где $\mathcal{R}$ - линейный псевдодифференциальный рекурсионный оператор, отображающий симметрию в новую симметрию. Рекурсионный оператор связан с бигамильтоновой структурой. В самом деле, если $\mathcal{H}_{1}$ и $\mathcal{H}_{2}-$ два совместных оператора Гамильтона, тогда $\mathcal{R}=\mathcal{H}_{1} \mathcal{H}_{2}^{-1}$ - рекурсионный оператор Нийенхейса [33], [11]. Достаточным для того, чтобы оператор $\mathcal{R}$ был рекурсионным для уравнения (3), является следующее условие [34]:

$$
D_{t}(\mathcal{R})=\left[\mathbf{F}_{*}, \mathcal{R}\right]
$$

где $\mathbf{F}_{*}$ - производная Фреше функции $\mathbf{F}$.

Похожее построение применимо и в дифференциально-разностном случае [13]. Подстановка выражения (39) в представление Дарбу-Лакса (11) приводит к уравнению

$$
D_{t_{k+1}}(M)=\mu(\lambda) D_{t_{k}}(M)-\mathcal{S}\left(B^{k}\right) M+M B^{k} .
$$

Уравнение (41) позволяет выразить элементы матрицы $B^{k}$ через $\mathbf{u}, \mathbf{u}_{t_{k}}, \mathbf{u}_{t_{k+1}}$ и их $\mathcal{S}$-сдвиги. Это, в свою очередь, позволяет найти линейный псевдоразностный оператор $\mathcal{R}$ такой, что $\mathbf{u}_{t_{k+1}}=\mathcal{R}\left(\mathbf{u}_{t_{k}}\right)$, т. е. рекурсионный оператор для дифференциально-разностйой иерархии коммутирующих симметрий. Как и в дифференциальном случае, если нам известно два совместных оператора Гамильтона $\mathcal{H}_{1}, \mathcal{H}_{2}$ или совместная пара гамильтониана $\mathcal{H}$ и симплектического оператора $\mathcal{J}$, тогда $\mathcal{R}=\mathcal{H}_{1} \mathcal{H}_{2}^{-1}$ и $\widetilde{\mathcal{R}}=\mathcal{H} \mathcal{J}$ являются рекурсионными операторами. Из этого построения следует, что матрица Дарбу $M$ и множитель $\mu(\lambda)$ полностью и однозначно определяют рекурсионный оператор.

В этом разделе мы поясним это построение на нескольких примерах. В разделе 4 мы представим обширный (но далеко не полный) список интегрируемых дифференциально-разностных уравнений с рекурсионными операторами, мультигамильтоновой структурой и представлениями Дарбу-Лакса.

3.1. Дифференциально-разностные уравнения, получаемые из нелинейного уравнения Шредингера. Поясним наши построения с помощью матриц Дарбу $M$ и $N(18)$, связанных с нелинейным уравнением Шредингера. В этом случае множитель $\mu(\lambda)=\lambda(15)$ и матрица $B^{k}$ не зависит от спектрального параметра $\lambda$.

Построим рекурсионный оператор для цепочки Тоды (20) с помощью матрицы Дарбу $N(22)$ и множителя $\mu(\lambda)=\lambda$. Подставим

$$
B=\left(\begin{array}{cc}
a & b \\
c & -a
\end{array}\right)
$$


в уравнение

$$
D_{t_{k+1}}(N)=\lambda D_{t_{k}}(N)-\mathcal{S}(B) N+N B .
$$

Линейная по $\lambda$ часть уравнения (43) приводит к следующей системе уравнений:

$$
(\mathcal{S}-1) a=h_{t_{k}}, \quad b=-\phi_{t_{k}} e^{\phi}, \quad c_{1}=-\phi_{t_{k}} e^{-\phi} .
$$

Таким образом, мы можем найти все элементы $a=(\mathcal{S}-1)^{-1} h_{t_{k}}, b=-e^{\phi} \phi_{t_{k}}, c=$ $-e^{-\phi_{-1}} \mathcal{S}^{-1} \phi_{t_{k}}$ матрицы $B$. Тогда не зависящая от $\lambda$ часть уравнения (43) сводится к уравнению

$$
\begin{aligned}
\phi_{t_{k+1}} & =b h-a-a_{1}=-(\mathcal{S}+1)(\mathcal{S}-1)^{-1} h_{t_{k}}-h \phi_{t_{k}}, \\
h_{t_{k+1}} & =\left(a-a_{1}\right) h+c e^{\phi}-b_{1} e^{-\phi}=-h h_{t_{k}}-e^{\phi-\phi-1} \mathcal{S}^{-1} \phi_{t_{k}}+e^{\phi_{1}-\phi} \mathcal{S} \phi_{t_{k}},
\end{aligned}
$$

которое можно представить в виде рекурсии

$$
\left(\begin{array}{l}
\phi_{t_{k+1}} \\
h_{t_{k+1}}
\end{array}\right)=\mathcal{R}\left(\begin{array}{l}
\phi_{t_{k}} \\
h_{t_{k}}
\end{array}\right)
$$

с псевдоразностным оператором

$$
\mathcal{R}=\left(\begin{array}{cc}
-h & -(\mathcal{S}+1)(\mathcal{S}-1)^{-1} \\
e^{\phi_{1}-\phi} \mathcal{S}-e^{\phi-\phi_{-1}} \mathcal{S}^{-1} & -h
\end{array}\right) .
$$

Иерархию симметрий (21) можно породить рекуррентным образом, начиная с затравочной симметрии $\phi_{t_{0}}=-2, h_{t_{0}}=0$.

Как и в случае скалярных дискретных уравнений [11], [35], канонический ряд плотностей локальных законов сохранения можно найти по вычетам $\rho_{k}=\operatorname{res} \mathcal{R}^{k}$. Вычет $\operatorname{res} \mathcal{A}$ для матричного псевдоразностного оператора $\mathcal{A}$ находится следующим образом. Любой псевдоразностный оператор $\mathcal{A}$ однозначно представим своим рядом Лорана

$$
\mathcal{A}=\sum_{k=0}^{\infty} \mathcal{A}^{m-k} \mathcal{S}^{m-k}
$$

причем вычеты вычисляются по следующей формуле: $\operatorname{res} \mathcal{A}=\operatorname{trace}\left(\mathcal{A}^{0}\right)$. Например, перепишем рекурсионный оператор (44) в виде

$$
\mathcal{R}=\left(\begin{array}{cc}
0 & 0 \\
e^{\phi_{1}-\phi} & 0
\end{array}\right) \mathcal{S}+\left(\begin{array}{cc}
-h & -1 \\
0 & -h
\end{array}\right)+\left(\begin{array}{cc}
0 & -2 \\
-e^{\phi-\phi_{-1}} & 0
\end{array}\right) \mathcal{S}^{-1}+\cdots
$$

Следовательно, величины

$$
\rho_{1}=\operatorname{res} \mathcal{R}=-2 h, \quad \rho_{2}=\operatorname{res} \mathcal{R}^{2}=2 h^{2}-2 e^{\phi_{1}-\phi}-2 e^{\phi-\phi_{-1}}, \quad \ldots
$$

являются сохраняющимися плотностями для цепочки Тоды (20). В самом деле, имеем

$$
\begin{aligned}
& D_{x} \rho_{1}=-4(\mathcal{S}-1) e^{\phi-\phi_{-1}}, \\
& D_{x} \rho_{2}=4(\mathcal{S}-1)\left(e^{\phi-\phi_{-1}}\left(h_{-1}+h\right)\right) .
\end{aligned}
$$


Теперь возьмем матрицу Дарбу $M(18)$ и матрицу $B$ вида (42). Из линейной по $\lambda$ части уравнения

$$
D_{t_{k+1}}(M)=\lambda D_{t_{k}}(M)-\mathcal{S}(B) M+M B
$$

следует, что

$$
a=(\mathcal{S}-1)^{-1} f_{t_{k}}, \quad b=-p_{t_{k}}, \quad c=q_{t_{k}} .
$$

Не зависящая от $\lambda$ часть уравнения (45) приводит к рекуррентному соотношению для системы $(23)$

$$
\left(\begin{array}{l}
f_{t_{k+1}} \\
p_{t_{k+1}} \\
q_{t_{k+1}}
\end{array}\right)=\left(\begin{array}{c}
-f f_{t_{k}}+\mathcal{S}\left(q p_{t_{k}}\right)+p q_{t_{k}} \\
-2 p(\mathcal{S}-1)^{-1} f_{t_{k}}-f_{t_{k}} p-f p_{t_{k}}+\mathcal{S}\left(p_{t_{k}}\right) \\
2 q(\mathcal{S}-1)^{-1} f_{t_{k}}-q \mathcal{S}^{-1}\left(f_{t_{k}}\right)+\mathcal{S}^{-1} q_{t_{k}}-f_{-1} q_{t_{k}}
\end{array}\right)=\mathcal{R}\left(\begin{array}{c}
f_{t_{k}} \\
p_{t_{k}} \\
q_{t_{k}}
\end{array}\right)
$$

где псевдоразностный рекурсионный оператор имеет вид

$$
\mathcal{R}=\left(\begin{array}{ccc}
-f & q_{1} \mathcal{S} & p \\
-p & -f+\mathcal{S} & 0 \\
-q \mathcal{S}^{-1} & 0 & -f_{-1}+\mathcal{S}^{-1}
\end{array}\right)+\left(\begin{array}{c}
0 \\
-2 p \\
2 q
\end{array}\right)(\mathcal{S}-1)^{-1}\left(\begin{array}{lll}
1 & 0 & 0
\end{array}\right)
$$

Используя первый интеграл системы (23) и исключая $f_{k}=p_{k} q_{k+1}+\alpha_{k}$, получим соответствующее рекуррентное соотношение и оператор для системы (25) в виде

$$
\left(\begin{array}{c}
p_{t_{k+1}} \\
q_{t_{k+1}}
\end{array}\right)=\mathcal{R}^{\prime}\left(\begin{array}{c}
p_{t_{k}} \\
q_{t_{k}}
\end{array}\right)
$$

где

$$
\mathcal{R}^{\prime}=\left(\begin{array}{cc}
\mathcal{S}-2 p q_{1}-\alpha & -p^{2} \mathcal{S}-2 p p_{-1} \\
-q^{2} \mathcal{S}^{-1} & \mathcal{S}^{-1}-\alpha_{-1}
\end{array}\right)+\left(\begin{array}{c}
-2 p \\
2 q
\end{array}\right)(\mathcal{S}-1)^{-1}\left(q_{1}, \quad p_{-1}\right) .
$$

Как отмечалось, уравнение (25) связано с цепочкой Мерола-Раниско-Ту. Поэтому можно убедиться, что тот же самый оператор получается из приведенного в п. 4.10 рекурсионного оператора для цепочки Мерола-Раниско-Ту с помощью формулы (58).

\section{2. Разностное уравнение, соответствующее диэдральной группе ре-} дукций. Покажем, как вычислить рекурсионный оператор для системы (37), представление Лакса которой инвариантно относительно диэдральной группы редукций.

После простой замены переменных $p \rightarrow p-1, q \rightarrow q-1$ система (37) приобретает вид

$$
p_{x}=2 p(\mathcal{S}-1)(2 q-p q), \quad q_{1, x}=2 q_{1}(\mathcal{S}-1)(2 p-p q) .
$$

Эти уравнения приводятся к релятивистской цепочке Вольтерра, описанной в п. 4.9, после применения масштабного преобразования

$$
p=-2 v, \quad q=-2 u, \quad x=-\frac{t}{4} .
$$

Выпишем соответствующие матрицу Дарбу и множитель:

$$
M=f\left(\begin{array}{cc}
\lambda^{2}+1-p-q_{1} & \lambda(p-1)+\lambda^{-1}\left(q_{1}-1\right) \\
\lambda\left(q_{1}-1\right)+\lambda^{-1}(p-1) & \lambda^{-2}+1-p-q_{1}
\end{array}\right), \quad \mu(\lambda)=\frac{1}{2}\left(\lambda^{2}+\lambda^{-2}\right) .
$$


Этой информации достаточно для нахождения рекурсионного оператора, самой системы (48) и иерархии ее локальных симметрий. Из

$$
\operatorname{det} M=-\left(\lambda-\lambda^{-1}\right)^{2} f^{2} p q_{1}
$$

следует, что $f^{2} p q_{1}$ не зависит от $x$ (теорема Абеля). Таким образом, можно положить $f=\left(p q_{1}\right)^{-1 / 2}$.

Выражение (31) мотивирует представление

$$
B=a \mathbf{a}_{1}+b \mathbf{a}_{2}+c \mathbf{a}_{3}
$$

где коэффициенты $a, b, c$ не зависят от $\lambda$. Теперь в уравнении (41) левая и правая части - рациональные матричные функции от $\lambda$. Левая часть имеет только простые полюсы. Требование равенства нулю коэффициента при полюсе третьего порядка $\lambda^{-3}$ в правой части эквивалентно системе

$$
\begin{aligned}
a & =\frac{1}{2}\left(c+c_{1}\right)(p-1)+\frac{(p-1) p \mathcal{S} q_{t_{k}}-(p+1) q_{1} p_{t_{k}}}{4 p q_{1}}, \\
b_{1} & =\frac{1}{2}\left(c+c_{1}\right)\left(q_{1}-1\right)+\frac{\left(q_{1}+1\right) p \mathcal{S} q_{t_{k}}+\left(1-q_{1}\right) q_{1} p_{t_{k}}}{4 p q_{1}} .
\end{aligned}
$$

Полюсы второго порядка исчезают при условии

$$
c-c_{1}=\frac{q_{1} p_{t_{k}}+p q_{1, t_{k}}}{2 p q_{1}} .
$$

Теперь, используя (52), можно упростить (51), исключая $c_{1}$ и $c_{-1}$ из $a, b$ :

$$
\begin{aligned}
& a=c(p-1)-\frac{1}{2} p_{t_{k}}, \\
& b=c(q-1)+\frac{1}{2} q_{t_{k}} .
\end{aligned}
$$

Вычеты в левой и правой частях (41) приводят к уравнениям

$$
\begin{aligned}
& p_{t_{k+1}}=c p(\mathcal{S}-1)(p q-2 q)+\left(p-q_{1}-1-\frac{1}{2} p_{1} q_{1}\right) p_{t_{k}}-\frac{1}{2} p q_{1} \mathcal{S} p_{t_{k}}+ \\
&+\left(p-\frac{1}{2} p^{2}\right) q_{t_{k}}+\left(p-\frac{1}{2} p p_{1}\right) \mathcal{S} q_{t_{k}} \\
& q_{t_{k+1}}=c q(\mathcal{S}-1)\left(p_{-1} q_{-1}-2 p_{-1}\right)+\left(q+p_{-1}-1-\frac{1}{2} p_{-1} q_{-1}\right) q_{t_{k}}- \\
&-\frac{1}{2} p_{-1} q \mathcal{S}^{-1} q_{t_{k}}+\left(q-\frac{1}{2} q^{2}\right) p_{t_{k}}+\left(q-\frac{1}{2} q q_{-1}\right) \mathcal{S}^{-1} p_{t_{k}}
\end{aligned}
$$

Не зависящая от $\lambda$ часть уравнения (41) автоматически удовлетворяется после подстановки выражений (52)-(54) в уравнение (41). 
Формулы (54) вместе с (52) представляют собой рекуррентные соотношения. Дифференциально-разностное уравнение (затравочную симметрию) $p_{x}=2 K_{p}^{(1)}$, $q_{x}=2 K_{q}^{(1)}(48)$ можно восстановить из этой рекурсии, взяв производную правых частей уравнений (54) по $c$, т. е.

$$
\left(K_{p}^{(1)}, K_{q}^{(1)}\right)=\left(p(\mathcal{S}-1)(p q-2 q), q(\mathcal{S}-1)\left(p_{-1} q_{-1}-2 p_{-1}\right)\right) .
$$

Это не удивительно. В самом деле, чтобы решить уравнение (52) относительно $c$, нужно обратить разностный оператор $\mathcal{S}-1$, ядро которого представляет собой поле констант. В результате вектор $p_{x}, q_{x}$ может внести свой вклад в $p_{t_{k+1}}, q_{t_{k+1}}$ с произвольным постоянным коэффициентом.

Теперь выпишем явно рекурсионный оператор, соответствующий рекурсии (54), в виде

$$
\begin{aligned}
& \mathcal{R}=\left(\begin{array}{cc}
p+q_{1}-1-\frac{1}{2} p_{1} q_{1}-\frac{1}{2} p q_{1} \mathcal{S} & p-\frac{1}{2} p^{2}+\left(p-\frac{1}{2} p p_{1}\right) \mathcal{S} \\
q-\frac{1}{2} q^{2}+\left(q-\frac{1}{2} q q_{-1}\right) \mathcal{S}^{-1} & q+p_{-1}-1-\frac{1}{2} p_{-1} q_{-1}-\frac{1}{2} p_{-1} q \mathcal{S}^{-1}
\end{array}\right)-
\end{aligned}
$$

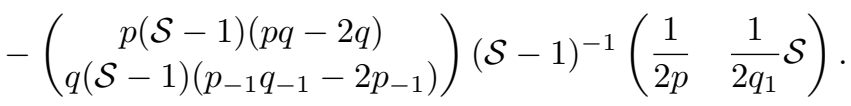

Очевидно, что $\mathcal{R}+I$, где $I$ - единичная $(2 \times 2)$-матрица, также является рекурсионным оператором, который можно восстановить с помощью преобразования (49) по рекурсионному оператору для релятивистской цепочки Вольтерра, представленной в п. 4.9 .

\section{4. СПИСОК ИНТЕГРИРУЕМЫХ ДИФФЕРЕНЦИАЛЬНО-РАЗНОСТНЫХ УРАВНЕНИЙ}

В этом разделе мы приведем длинный список интегрируемых дифференциально-разностных уравнений вместе с их гамильтоновыми структурами, рекурсионными операторами, нетривиальными обобщенными симметриями и представлениями Лакса. Для полноты изложения мы введем обозначения и напомним некоторые определения объектов нашего списка через производные Ли. Теоретическую основу и подробные определения гамильтоновых и симплектических операторов можно найти в монографиях [36], [37]. Здесь же мы хотели бы отметить недавние результаты в теории нелокальных гамильтоновых структур для нелинейных уравнений в частных производных [38].

Пусть $\mathbf{u}=\left(u^{1}, \ldots, u^{N}\right)$ - вектор-функция переменной $n \in \mathbb{Z}$ и времени $t$. Эволюционное дифференциально-разностное уравнение для зависимой переменной $\mathbf{u}$ имеет вид

$$
\mathbf{u}_{t}=\mathbf{K}[\mathbf{u}],
$$

где $\mathbf{K}[\mathbf{u}]$ означает, что гладкая вектор-функция $\mathbf{K}$ зависит от $\mathbf{u}$ и от конечного количества ее сдвигов $\mathbf{u}_{i}=\mathcal{S}^{i} \mathbf{u}$. Во всех наших примерах, после соответствующей точечной замены переменных, можно считать $\mathbf{K}[\mathbf{u}] \in \mathcal{F}^{N}$, где $\mathcal{F}=(\mathbb{C}, \mathbf{u}, \mathcal{S})$ - разностное поле рациональных функций от $\left\{u_{k}^{i} \mid k \in \mathbb{Z}, i=1, \ldots, N\right\}$. Производную 
Фреше $a_{\star}$ от $a \in \mathcal{F}$ определим в виде строки разностных операторов:

$$
a_{\star}=\sum_{k \in \mathbb{Z}}\left(\frac{\partial a}{\partial u_{k}^{1}}, \ldots, \frac{\partial a}{\partial u_{k}^{N}}\right) \mathcal{S}^{k},
$$

таким образом, производная Фреше от $\mathbf{K}[\mathbf{u}]$ - это разностный оператор с коэффициентами в виде квадратных матриц, причем элементы матричных коэффициентов являются элементами из поля $\mathcal{F}$.

Вариационная производная функции $a \in \mathcal{F}$ представляет собой столбец:

$$
\delta_{\mathbf{u}}(a):=a_{\star}^{\dagger}(1)=\left(\frac{\partial}{\partial u^{1}}, \ldots, \frac{\partial}{\partial u^{N}}\right)^{\mathrm{T}} \sum_{k \in \mathbb{Z}} \mathcal{S}^{k}(a) .
$$

Если уравнение (56) гамильтоново, то оно имеет вид

$$
\mathbf{u}_{t}=\mathcal{H}\left(\delta_{\mathbf{u}}(f)\right)
$$

где $\mathcal{H}$ - гамильтонов (псевдо)разностный оператор, а $f$ - гамильтониан системы.

ОПРЕДЕЛЕНИЕ 1. Для данного дифференциально-разностного эволюционного уравнения (56) имеют место следующие утверждения:

1) $\mathbf{G}$ является его симметрией, если $L_{\mathbf{K}} \mathbf{G}:=[\mathbf{K}, \mathbf{G}]:=\mathbf{G}_{\star}(\mathbf{K})-\mathbf{K}_{\star}(\mathbf{G})=0$;

2) $\mathcal{H}$ является его гамильтоновым оператором, если гамильтонов оператор $\mathcal{H}$ удовлетворяет условию $L_{\mathbf{K}} \mathcal{H}:=\mathcal{H}_{\star}[\mathbf{K}]-\mathbf{K}_{\star} \mathcal{H}-\mathcal{H} \mathbf{K}_{\star}^{\dagger}=0$;

3) $\mathcal{J}$ является его симплектическим оператором, если симплектический оператор $\mathcal{J}$ удовлетворяет условию $L_{\mathbf{K}} \mathcal{J}:=\mathcal{J}_{\star}[\mathbf{K}]+\mathbf{K}_{\star}^{\dagger} \mathcal{J}+\mathcal{J} \mathbf{K}_{\star}=0$;

4) $\mathcal{R}$ является его рекурсионным оператором, если $L_{\mathbf{K}} \mathcal{R}:=\mathcal{R}_{\star}[\mathbf{K}]-\mathbf{K}_{\star} \mathcal{R}+$ $\mathcal{R} \mathbf{K}_{\star}=0$.

Здесь $L_{\mathbf{K}}$ означает производную Ли, 夫 означает производную Фреше, a $\dagger$ означает формальное сопряжение разностного оператора.

Если симметрия уравнения (56) явно зависит от $\mathbf{u}_{i}$, где $i \neq 0$, мы будем называть ее обобщенной симметрией. Уравнение интегрируемо, если у него есть бесконечно много обобщенных симметрий, зависящих от конечных наборов переменных $\mathbf{u}_{i}$, размеры которых увеличиваются. Симметрии интегрируемых систем можно получить с помощью рекурсионного оператора, который часто является нелокальным. Достаточное условие, налагаемое на нелокальные псевдоразностные рекурсионные операторы и гарантирующее порождение бесконечной иерархии коммутирующих локальных симметрий, обсуждалось в работе [11]. В нашем списке мы также приводим частные результаты, относящиеся к мастер-симметриям (более подробное описание мастер-симметрий приведено в работах [39], [40]).

Теперь посмотрим, как изменяются рекурсионный, гамильтонов и симплектический операторы при преобразованиях (разностных подстановках). Если эволюционное разностное уравнение (56) с помощью разностной подстановки $\mathbf{u}=\mathbf{F}[\mathbf{v}]$, связано с другим уравнением вида

$$
\mathbf{v}_{t}=\mathbf{G}[\mathbf{v}]
$$


то рекурсионный, гамильтонов и симплектический операторы $\widehat{\mathcal{R}}, \widehat{\mathcal{H}}$ и $\widehat{\mathcal{J}}$ для уравнения (57) можно выразить через соответствующие операторы $\mathcal{R}, \mathcal{H}$ и $\mathcal{J}$ уравнения (56):

$$
\widehat{\mathcal{R}}=\left.\mathbf{F}_{\star}^{-1} \circ \mathcal{R}\right|_{\mathbf{u}=\mathbf{F}[\mathbf{v}]} \circ \mathbf{F}_{\star}, \quad \widehat{\mathcal{H}}=\left.\mathbf{F}_{\star}^{-1} \circ \mathcal{H}\right|_{\mathbf{u}=\mathbf{F}[\mathbf{v}]^{\circ}} \mathbf{F}_{\star}^{\dagger}-1, \quad \widehat{\mathcal{J}}=\left.\mathbf{F}_{\star}^{\dagger} \circ \mathcal{J}\right|_{\mathbf{u}=\mathbf{F}[\mathbf{v}]} \circ \mathbf{F}_{\star},
$$

где о означает композицию операторов.

ПримеР 1. Известно, что уравнение Вольтерра $u_{t}=u\left(u_{1}-u_{-1}\right)$ (см. п. 4.1) связано с модифицированным уравнением Вольтерра

$$
v_{t}=v^{2}\left(v_{1}-v_{-1}\right)
$$

с помощью разностной подстановки (преобразование типа Миуры) $u=F[v]=v v_{1}$. Оператор $\mathcal{H}_{1}=u\left(\mathcal{S}-\mathcal{S}^{-1}\right) u$ - гамильтонов оператор для уравнения Вольтерра. Отметим, что производная Фреше для $v v_{1}$ имеет вид $F_{\star}=v_{1}+v \mathcal{S}$, при этом $F_{\star}^{-1} u=$ $v(1+\mathcal{S})^{-1}$ и $u F_{\star}^{\dagger}=\mathcal{S}(1+\mathcal{S})^{-1} v$. Теперь можно найти гамильтонов оператор $\widehat{\mathcal{H}}_{1}$ для модифицированного уравнения Вольтерра (59):

$$
\begin{aligned}
\widehat{\mathcal{H}}_{1} & =\left(v_{1}+v \mathcal{S}\right)^{-1} v v_{1}\left(\mathcal{S}-\mathcal{S}^{-1}\right) v v_{1}\left(v_{1}+v \mathcal{S}\right)^{-1}= \\
& =v(1+\mathcal{S})^{-1}\left(\mathcal{S}^{2}-1\right)(\mathcal{S}+1)^{-1} v=v(\mathcal{S}-1)(\mathcal{S}+1)^{-1} v .
\end{aligned}
$$

Тем же способом можно посчитать второй гамильтонов оператор $\widehat{\mathcal{H}}_{2}$ и рекурсионный оператор $\widehat{\mathcal{R}}$ для модифицированного уравнения Вольтерра (ср. с п. 4.2).

Как уже обсуждалось в разделе 2, совместность отображения Дарбу (8) с оператором Лакса (9)

$$
\mathcal{S}(\Phi)=M \Phi, \quad D_{t}(\Phi)=U(\mathbf{u} ; \lambda) \Phi
$$

приводит к уравнению (10)

$$
D_{t}(M)=\mathcal{S}(U) M-M U
$$

которое эквивалентно интегрируемой системе дифференциально-разностных уравнений. В литературе условие совместности (61) часто называют представлением нулевой кривизны или представлением Лакса уравнения (56). Поскольку оно включает в себя матрицу Дарбу и оператор Лакса, было бы более естественно называть его представлением Дарбу-Лакса. Однако мы все-таки будем называть его представлением Лакса в соответствии с принятой терминологией. В последующем списке мы просто приводим выражения для обеих матриц $M$ и $U$ представления Лакса.

Существует множество работ, в которых интегрируемая система возникает как условие совместности двух линейных задач со скалярными линейными разностными операторами $L$ и $A$

$$
L \phi=\lambda \phi, \quad \phi_{t}=A \phi,
$$

где $\phi$ - собственная функция оператора $L$, соответствующая собственному значению $\lambda$ и $\lambda_{t}=0$. Уравнение (56) эквивалентно их условию совместности

$$
D_{t}(L)=[A, L]=A L-L A .
$$


Этот подход похож на первоначальное определение Лакса, в котором дифференциальные операторы просто заменяются на разностные. В теории обыкновенных дифференциальных уравнений скалярные уравнения высокого порядка можно представить в виде системы уравнений первого порядка. То же самое можно сделать и в случае скалярных разностных уравнений высокого порядка, представив их в виде системы разностных уравнений первого порядка. Таким способом можно переписать скалярное представление (62), (63) в виде матричного представления Дарбу-Лакса первого порядка (60), (61).

Пример 2. Для цепочки Вольтерра, приведенной в п. 4.1, матричное представление Лакса получается из его скалярного представления Лакса с $L=\mathcal{S}+u \mathcal{S}^{-1}$ и $A=\mathcal{S}^{2}+u_{1}+u$.

Пусть $\Phi=\left(\phi^{1}, \phi^{2}\right)^{\mathrm{T}}=\left(\phi,-\mathcal{S}^{-1} \phi\right)^{\mathrm{T}}$. Можно переписать уравнение $L \phi=\lambda \phi$ системы (62) в виде

$$
\mathcal{S}(\Phi)=\mathcal{S}\left(\begin{array}{c}
\phi \\
-\phi_{-1}
\end{array}\right)=\left(\begin{array}{c}
\mathcal{S} \phi \\
-\phi
\end{array}\right)=\left(\begin{array}{cc}
\lambda & u \\
-1 & 0
\end{array}\right)\left(\begin{array}{c}
\phi \\
-\phi_{-1}
\end{array}\right),
$$

т. е. $\mathcal{S}\left(\phi^{1}\right)=\lambda \phi^{1}+u \phi^{2}$ и $\mathcal{S}\left(\phi^{2}\right)=-\phi^{1}$. Теперь уравнение $\phi_{t}=A \phi$ представимо в виде

$$
\begin{aligned}
\phi_{t}^{1} & =\mathcal{S}^{2} \phi^{1}+\left(u_{1}+u\right) \phi^{1}=\mathcal{S}\left(\lambda \phi^{1}+u \phi^{2}\right)+\left(u_{1}+u\right) \phi^{1}= \\
& =\lambda \mathcal{S} \phi^{1}+u \phi^{1}=\left(\lambda^{2}+u\right) \phi^{1}+\lambda u \phi^{2}, \\
\phi_{t}^{2} & =-\mathcal{S}^{-1} \phi_{t}^{1}=-\left(\lambda \phi^{1}+u \phi^{2}+\left(u_{-1}+u\right) \mathcal{S}^{-1} \phi^{1}\right)=-\lambda \phi^{1}+u_{-1} \phi^{2} .
\end{aligned}
$$

Таким образом, имеем

$$
D_{t}(\Phi)=\left(\begin{array}{c}
\phi_{t}^{1} \\
\phi_{t}^{2}
\end{array}\right)=\left(\begin{array}{cc}
\lambda^{2}+u & \lambda u \\
-\lambda & u_{-1}
\end{array}\right)\left(\begin{array}{l}
\phi^{1} \\
\phi^{2}
\end{array}\right) .
$$

В нашем списке мы приводим либо скалярное, либо матричное представление Лакса. Необходимо помнить, что:

1) соотношения между нелокальными или псевдоразностными операторами, например (67), следует понимать как тождества в некоммутативном кольце псевдоразностных рядов Лорана;

2) при расчетах с псевдоразностными операторами часто используются тождества, похожие на интегрирование по частям:

$$
(\mathcal{S}-1)^{-1}\left(f_{1}-f\right)(\mathcal{S}-1)^{-1}=f(\mathcal{S}-1)^{-1}-(\mathcal{S}-1)^{-1} f_{1}
$$

3) поскольку для любой константы $c \in \mathbb{C}$ справедливо $(\mathcal{S}-1) c=0$, то действие операторов, содержащих $(\mathcal{S}-1)^{-1}$, определено неоднозначно. Соответствующие результаты приведены в списке с точностью до этих "констант интегрирования".

\section{1. Цепочка Вольтерра.}

- Уравнение [10]:

$$
u_{t}=u\left(u_{1}-u_{-1}\right) .
$$

- Гамильтонова структура [41], [42]: $u_{t}=H_{i} \delta_{u} f_{i}$,

$$
\mathcal{H}_{1}=u\left(\mathcal{S}-\mathcal{S}^{-1}\right) u, \quad f_{1}=u,
$$




$$
\mathcal{H}_{2}=u\left(\mathcal{S} u \mathcal{S}+u \mathcal{S}+\mathcal{S} u-u \mathcal{S}^{-1}-\mathcal{S}^{-1} u-\mathcal{S}^{-1} u \mathcal{S}^{-1}\right) u, \quad f_{2}=\frac{1}{2} \ln u
$$

- Рекурсионный оператор:

$$
\begin{aligned}
\mathcal{R} & =\mathcal{H}_{2} \mathcal{H}_{1}^{-1}=u \mathcal{S}+u+u_{1}+u \mathcal{S}^{-1}+u\left(u_{1}-u_{-1}\right)(\mathcal{S}-1)^{-1} \frac{1}{u}= \\
& =u\left(\mathcal{S}-\mathcal{S}^{-1}\right) u\left(\frac{1}{u}(\mathcal{S}-1)^{-1}+\mathcal{S}(\mathcal{S}-1)^{-1} \frac{1}{u}\right) .
\end{aligned}
$$

- Нетривиальная симметрия [42], [43]:

$$
\mathcal{R}\left(u_{t}\right)=u\left(u_{1} u_{2}+u_{1}^{2}+u u_{1}-u u_{-1}-u_{-1}^{2}-u_{-1} u_{-2}\right) .
$$

- Мастер-симметрия [42], [39], [44]:

$$
\mathcal{R}(u)=n u_{t}+u\left(2 u_{1}+u+u_{-1}\right) .
$$

- Представление Лакса [45]:

$$
L=\mathcal{S}+u \mathcal{S}^{-1}, \quad A=\mathcal{S}^{2}+u_{1}+u,
$$

его можно также представить в матричной форме:

$$
M=\left(\begin{array}{cc}
\lambda & u \\
-1 & 0
\end{array}\right), \quad U=\left(\begin{array}{cc}
\lambda^{2}+u & \lambda u \\
-\lambda & u_{-1}
\end{array}\right)
$$

Это уравнение также известно как модель Лотки-Вольтерра, цепочка Каца-ван Мербеке или цепочка Ленгмюра [46]. Так называемое уравнение Каца- ван МербекеЛенгмюра [41]

$$
w_{\tau}=w\left(w_{1}^{\epsilon}-w_{-1}^{\epsilon}\right), \quad \epsilon=\text { const }, \quad \epsilon \neq 0,
$$

связано с уравнением (64) точечным преобразованием $u=w^{\epsilon}$ и $t=\epsilon \tau$. Это уравнение представимо в виде уравнения

$$
w_{t}=e^{w+w_{1}}-e^{w+w_{-1}},
$$

которое преобразуется в уравнение (64) с помощью подстановки $u=e^{w+w_{1}}$.

\section{2. Модифицированное уравнение Вольтерра.}

- Уравнение [47], [43]:

$$
u_{t}=u^{2}\left(u_{1}-u_{-1}\right) .
$$

- Гамильтонова структура [48], [43]:

$$
\begin{array}{llrl}
\mathcal{H}_{1} & =u(\mathcal{S}-1)(\mathcal{S}+1)^{-1} u, & & f_{1}=u u_{1}, \\
\mathcal{H}_{2} & =u^{2}\left(\mathcal{S}-\mathcal{S}^{-1}\right) u^{2}, & & f_{2}=\ln u .
\end{array}
$$

- Рекурсионный оператор:

$$
\mathcal{R}=\mathcal{H}_{2} \mathcal{H}_{1}^{-1}=u^{2} \mathcal{S}+2 u u_{1}+u^{2} \mathcal{S}^{-1}+2 u^{2}\left(u_{1}-u_{-1}\right)(\mathcal{S}-1)^{-1} \frac{1}{u} .
$$


- Нетривиальная симметрия [43]:

$$
\mathcal{R}\left(u_{t}\right)=u^{2} u_{1}^{2}\left(u_{2}+u\right)-u^{2} u_{-1}^{2}\left(u+u_{-2}\right) .
$$

- Мастер-симметрия [43]:

$$
\mathcal{R}\left(\frac{u}{2}\right)=n u_{t}+\frac{u^{2}}{2}\left(3 u_{1}+u_{-1}\right) .
$$

- Представление Лакса [26]:

$$
M=\left(\begin{array}{cc}
0 & u \\
-u & \lambda
\end{array}\right), \quad U=\left(\begin{array}{cc}
u u_{-1} & \lambda u_{-1} \\
-\lambda u & \lambda^{2}+u u_{-1}
\end{array}\right) .
$$

Модифицированное уравнение Вольтерра также известно как дискретное модифицированное уравнение Кортевега-де Фриза. С помощью преобразования Миуры $w=u u_{1}$ оно сводится к цепочке Вольтерра $w_{t}=w\left(w_{1}-w_{-1}\right)$, приведенной в п. 4.1.

\section{3. Дискретизация Ямилова уравнения Кричевера-Новикова.}

- Уравнение [49] (V4, $\nu=0$ в п. 4.4):

$$
u_{t}=\frac{R\left(u_{1}, u, u_{-1}\right)}{u_{1}-u_{-1}}:=K^{(1)},
$$

где $R$ - полином с постоянными коэффициентами $\alpha, \beta, \gamma, \delta, \epsilon \in \mathbb{C}$, заданный выражением

$$
R(u, v, w)=\left(\alpha v^{2}+2 \beta v+\gamma\right) u w+\left(\beta v^{2}+\lambda v+\delta\right)(u+w)+\gamma v^{2}+2 \delta v+\epsilon .
$$

- Две нетривиальные симметрии [50]-[53], [35], [11]:

$$
\begin{aligned}
K^{(2)}= & \frac{R\left(u, u_{-1}, u\right) R\left(u_{1}, u, u_{1}\right)}{\left(u_{1}-u_{-1}\right)^{2}}\left(\frac{1}{u_{2}-u}+\frac{1}{u-u_{-2}}\right), \\
K^{(3)}= & \frac{R\left(u_{1}, u, u_{1}\right) R\left(u, u_{-1}, u\right)}{\left(u_{1}-u_{-1}\right)^{2}}\left(\frac{\mathcal{S}^{2} K^{(1)}}{\left(u_{2}-u\right)^{2}}+\frac{\mathcal{S}^{-2} K^{(1)}}{\left(u-u_{-2}\right)^{2}}\right)+ \\
& +K^{(1)} K^{(2)}\left(\frac{1}{u_{2}-u}+\frac{1}{u-u_{-2}}\right) .
\end{aligned}
$$

- Гамильтонова структура [11], [53]:

$$
\begin{aligned}
& \mathcal{H}= A \mathcal{S}-\mathcal{S}^{-1} A+2 K^{(1)}(\mathcal{S}-1)^{-1} \mathcal{S} K^{(2)}+2 K^{(2)}(\mathcal{S}-1)^{-1} K^{(1)}, \\
& \widehat{\mathcal{H}}=\widehat{A} \mathcal{S}^{2}-\mathcal{S}^{-2} \widehat{A}+\widehat{B} \mathcal{S}-\mathcal{S}^{-1} \widehat{B}+K^{(2)}(\mathcal{S}-1)^{-1}(\mathcal{S}+1) K^{(2)}+ \\
& \quad+2 K^{(1)}(\mathcal{S}-1)^{-1} \mathcal{S} K^{(3)}+2 K^{(3)}(\mathcal{S}-1)^{-1} K^{(1)},
\end{aligned}
$$

где

$$
\begin{aligned}
A & =\frac{R\left(u_{2}, u_{1}, u_{2}\right) R\left(u_{1}, u, u_{1}\right) R\left(u, u_{-1}, u\right)}{\left(u_{1}-u_{-1}\right)^{2}\left(u_{2}-u\right)^{2}} \\
\widehat{A} & =\frac{R\left(u_{3}, u_{2}, u_{3}\right) R\left(u_{2}, u_{1}, u_{2}\right) R\left(u_{1}, u, u_{1}\right) R\left(u, u_{-1}, u\right)}{\left(u_{1}-u_{-1}\right)^{2}\left(u_{2}-u\right)^{2}\left(u_{3}-u_{1}\right)^{2}}, \\
\widehat{B} & =2 A\left(\frac{K^{(1)}}{u-u_{-2}}-\frac{\partial_{u} R\left(u_{1}, u, u_{1}\right)}{2\left(u_{1}-u_{-1}\right)}+\frac{\partial^{2} R\left(u_{1}, u, u_{1}\right)}{4 \partial u \partial u_{1}}\right)+\frac{2 R\left(u, u_{-1}, u\right)}{\left(u_{1}-u_{-1}\right)^{2}} \mathcal{S}\left(K^{(1)} K^{(2)}\right) .
\end{aligned}
$$


- Симплектический оператор:

$$
\mathcal{J}=\frac{1}{R\left(u_{1}, u, u_{1}\right)} \mathcal{S}-\mathcal{S}^{-1} \frac{1}{R\left(u_{1}, u, u_{1}\right)} .
$$

- Рекурсионные операторы [11], [53]:

$$
\mathcal{R}=\mathcal{H} \mathcal{J} \quad \text { и } \quad \widehat{\mathcal{R}}=\widehat{\mathcal{H}} \mathcal{J} .
$$

Рекурсионные операторы $\mathcal{R}$ и $\widehat{\mathcal{R}}$ удовлетворяют алгебраическому уравнению

$$
\left(2 \widehat{\mathcal{R}}-I_{3}\right)^{2}=4\left(\mathcal{R}+I_{2}\right)^{3}-g_{2}\left(\mathcal{R}+I_{2}\right)-g_{3},
$$

где $I_{2}, I_{3}, g_{2}$ и $g_{3}$ - относительные и модулярные инварианты, связанные с $h=$ $R\left(u_{1}, u, u_{1}\right)$ и полиномом четвертой степени $f(u)=\left(\partial_{u_{1}} h\right)^{2}-2 h \partial_{u_{1}}^{2} h$ выражениями

$$
\begin{aligned}
g_{2} & =\frac{1}{48}\left(2 f f^{I V}-2 f^{\prime} f^{\prime \prime \prime}+\left(f^{\prime \prime}\right)^{2}\right) \\
g_{3} & =\frac{1}{3456}\left(12 f f^{\prime \prime} f^{I V}-9\left(f^{\prime}\right)^{2} f^{I V}-6 f\left(f^{\prime \prime \prime}\right)^{2}+6 f^{\prime} f^{\prime \prime} f^{\prime \prime \prime}-2\left(f^{\prime \prime}\right)^{3}\right), \\
I_{2} & =\frac{1}{6}\left(h \partial_{u}^{2} \partial_{u_{1}}^{2} h-\left(\partial_{u} h\right)\left(\partial_{u} \partial_{u_{1}}^{2} h\right)-\left(\partial_{u_{1}} h\right)\left(\partial_{u}^{2} \partial_{u_{1}} h\right)+\left(\partial_{u}^{2} h\right)\left(\partial_{u_{1}}^{2} h\right)\right)+\frac{1}{12}\left(\partial_{u} \partial_{u_{1}} h\right)^{2}, \\
I_{3} & =\frac{1}{4} \operatorname{det}\left(\begin{array}{ccc}
h & \partial_{u} h & \partial_{u}^{2} h \\
\partial_{u_{1}} h & \partial_{u_{1}} \partial_{u} h & \partial_{u_{1}} \partial_{u}^{2} h \\
\partial_{u_{1}}^{2} h & \partial_{u_{1}}^{2} \partial_{u} h & \partial_{u_{1}}^{2} \partial_{u}^{2} h
\end{array}\right) .
\end{aligned}
$$

4.4. Интегрируемые уравнения типа Вольтерра. Классификация интегрируемых уравнений типа Вольтерра вида

$$
u_{t}=f\left(u_{-1}, u, u_{1}\right),
$$

где $f$ - гладкая функция всех своих переменных, была проведена Ямиловым с использованием симметрийного подхода. В его знаменитой обзорной статье [43] приведен следующий полный список интегрируемых уравнений типа Вольтерра (вместе с законами сохранения высшего порядка) с точностью до точечных преобразований:

$\mathrm{V} 1: \quad u_{t}=P(u)\left(u_{1}-u_{-1}\right)$,

V2: $\quad u_{t}=P\left(u^{2}\right)\left(\frac{1}{u_{1}+u}-\frac{1}{u+u_{-1}}\right)$,

V3: $\quad u_{t}=Q(u)\left(\frac{1}{u_{1}-u}+\frac{1}{u-u_{-1}}\right)$,

$\mathrm{V} 4: \quad u_{t}=\frac{R\left(u_{1}, u, u_{-1}\right)+\nu R\left(u_{1}, u, u_{1}\right)^{1 / 2} R\left(u_{-1}, u, u_{-1}\right)^{1 / 2}}{u_{1}-u_{-1}}, \quad \nu \in\{0, \pm 1\}$,

V5: $\quad u_{t}=y\left(u_{1}-u\right)+y\left(u-u_{-1}\right), \quad y^{\prime}=P(y)$,

V6: $\quad u_{t}=y\left(u_{1}-u\right) y\left(u-u_{-1}\right)+\mu, \quad y^{\prime}=\frac{P(y)}{y}, \quad \mu \in \mathbb{C}$,

V7: $\quad u_{t}=\frac{1}{y\left(u_{1}-u\right)+y\left(u-u_{-1}\right)}+\mu, \quad y^{\prime}=P\left(y^{2}\right), \quad \mu \in \mathbb{C}$, 
V8: $\quad u_{t}=\frac{1}{y\left(u_{1}+u\right)-y\left(u+u_{-1}\right)}, \quad y^{\prime}=Q(y)$,

V9: $\quad u_{t}=\frac{y\left(u_{1}+u\right)-y\left(u+u_{-1}\right)}{y\left(u_{1}+u\right)+y\left(u+u_{-1}\right)}, \quad y^{\prime}=\frac{P\left(y^{2}\right)}{y}$,

V10: $\quad u_{t}=\frac{y\left(u_{1}+u\right)+y\left(u+u_{-1}\right)}{y\left(u_{1}+u\right)-y\left(u+u_{-1}\right)}, \quad y^{\prime}=\frac{Q(y)}{y}$,

V11: $\quad u_{t}=\frac{\left(1-y\left(u_{1}-u\right)\right)\left(1-y\left(u-u_{-1}\right)\right)}{y\left(u_{1}-u\right)+y\left(u-u_{-1}\right)}+\mu, \quad y^{\prime}=\frac{P\left(y^{2}\right)}{1-y^{2}}, \quad \mu \in \mathbb{C}$,

где $P$ и $Q$ - полиномы с постоянными коэффициентами $\alpha, \beta, \gamma, \delta, \epsilon$, имеющие вид

$$
\begin{aligned}
& P(u)=\alpha u^{2}+\beta u+\gamma, \\
& Q(u)=\alpha u^{4}+\beta u^{3}+\gamma u^{2}+\delta u+\epsilon,
\end{aligned}
$$

а полином $R$ задан выражением (66). Как утверждается в работе [43], проблема построения обобщенных симметрий для всех уравнений V1-V11 остается нерешенной, хотя известны мастер-симметрии для некоторых видов уравнений из этого списка [42], [39]. Известно, что преобразование Миуры $\tilde{u}=y\left(u_{1}-u\right)$ переводит уравнения V5 и V6 в V1, а уравнения V7 и V11 - в V2, а преобразование Миуры $\tilde{u}=y\left(u_{1}+u\right)$, в свою очередь, переводит уравнение V9 в V2, а уравнения V8 и V10 - в V3 [43]. Мы представим рекурсионные операторы, гамильтоновы операторы и мастер-симметрии для первых четырех уравнений. Соответствующие операторы для остальных уравнений можно получить с помощью преобразований Миуры.

4.4.1. Уравнение V1 (68).

- Гамильтонова структура:

$$
\mathcal{H}=P(u)\left(\mathcal{S}-\mathcal{S}^{-1}\right) P(u)
$$

- Симплектический оператор:

$\alpha\left(\mathcal{S}-\mathcal{S}^{-1}\right)+\left(\alpha u_{1}+\beta+\alpha u_{-1}\right) \mathcal{S}(\mathcal{S}-1)^{-1} \frac{P^{\prime}(u)}{P(u)}+\frac{P^{\prime}(u)}{P(u)}(\mathcal{S}-1)^{-1}\left(\alpha u_{1}+\beta+\alpha u_{-1}\right)$.

- Рекурсионный оператор:

$$
\mathcal{R}=P(u) \mathcal{S}+2 \alpha u u_{1}+\beta\left(u+u_{1}\right)+P(u) \mathcal{S}^{-1}+u_{t}(\mathcal{S}-1)^{-1} \frac{P^{\prime}(u)}{P(u)} .
$$

- Нетривиальная симметрия:

$\mathcal{R}\left(u_{t}\right)=P(u)\left(P\left(u_{1}\right) u_{2}+\alpha u u_{1}^{2}+\beta\left(u+u_{1}\right) u_{1}-P\left(u_{-1}\right) u_{-2}-\alpha u u_{-1}^{2}-\beta\left(u+u_{-1}\right) u_{-1}\right)$.

- Мастер-симметрия:

$$
\begin{array}{ll}
n u_{t}+P(u)\left(c u_{1}+\frac{\beta}{\alpha}+(2-c) u_{-1}\right), & c \in \mathbb{C}, \quad \text { при } \alpha \neq 0, \\
n u_{t}+P(u)\left(c u_{1}+u+(3-c) u_{-1}\right), & c \in \mathbb{C}, \quad \text { при } \alpha=0 .
\end{array}
$$


- Представление Лакса:

- Случай $\alpha=\beta=0$ соответствует линейному уравнению.

- Случай $\alpha=0, \beta \neq 0$ сводится к уравнению Вольтерра (64) линейной подстановкой $u \mapsto \beta^{-1}(u-\gamma)$. Таким образом, у него есть представление Лакса (65).

- В случае $\alpha \neq 0$ линейная подстановка $t \mapsto \alpha^{-1} t$ и $u \mapsto u-\beta / 2$ переводит полином $P(u)$ в уравнении V1 к виду $P(u)=u^{2}+c$, где $c=\gamma / \alpha-\beta^{2} / 4 \alpha^{2}$. Соответствующее представление Лакса имеет вид [26]

$$
M=\left(\begin{array}{cc}
c \lambda^{-1} & u \\
-u & \lambda
\end{array}\right), \quad U=\left(\begin{array}{cc}
c^{2} \lambda^{-2}+u u_{-1} & c \lambda^{-1} u+\lambda u_{-1} \\
-c \lambda^{-1} u_{-1}-\lambda u & \lambda^{2}+u u_{-1}
\end{array}\right)
$$

В это уравнение входят как цепочка Вольтерра из п. 4.1, так и модифицированная цепочка Вольтерра из п. 4.2. Что касается нелинейного уравнения, т. е. $\alpha \beta \neq 0$, его гамильтониан $f$ зависит от коэффициентов полинома $P$, заданного выражением (79). Если $\alpha \neq 0$, то возьмем $f=(1 / 2 \alpha) \ln P(u)$, если $\alpha=0$, то положим $f=u / \beta$.

Его гамильтонов оператор, симплектический оператор и рекурсионный оператор удовлетворяют следующему неожиданному соотношению:

$$
\mathcal{H} \mathcal{J}=\alpha \mathcal{R}^{2}+\beta^{2} \mathcal{R}+2 \gamma\left(\beta^{2}-2 \alpha \gamma\right)
$$

4.4.2. Уравнение V2 (69).

- Гамильтонова структура:

$$
\mathcal{H}=\frac{P\left(u^{2}\right)}{u_{1}+u} \mathcal{S} \frac{P\left(u^{2}\right)}{u+u_{-1}}-\frac{P\left(u^{2}\right)}{u+u_{-1}} \mathcal{S}^{-1} \frac{P\left(u^{2}\right)}{u_{1}+u}-u_{t}(\mathcal{S}+1)(\mathcal{S}-1)^{-1} u_{t} .
$$

- Симплектический оператор:

$$
\begin{aligned}
\mathcal{J}= & \frac{1}{\left(u+u_{1}\right)^{2}} \mathcal{S}-\mathcal{S}^{-1} \frac{1}{\left(u+u_{1}\right)^{2}}+\delta_{u} \rho(\mathcal{S}+1)(\mathcal{S}-1)^{-1} \delta_{u} \rho- \\
& -\left(\beta^{2}-4 \alpha \gamma\right) \frac{u}{P\left(u^{2}\right)}(\mathcal{S}-1)(\mathcal{S}+1)^{-1} \frac{u}{P\left(u^{2}\right)}
\end{aligned}
$$

где $\rho=(1 / 2) \ln \left(\left(u+u_{1}\right)^{2} / P\left(u^{2}\right)\right)$, и, таким образом,

$$
\delta_{u} \rho=\frac{1}{u_{1}+u}+\frac{1}{u+u_{-1}}-\frac{P\left(u^{2}\right)^{\prime}}{2 P\left(u^{2}\right)}, \quad P\left(u^{2}\right)^{\prime}=2 u\left(2 \alpha u^{2}+\beta\right) .
$$

- Рекурсионный оператор:

$$
\begin{gathered}
\mathcal{R}=\frac{P\left(u^{2}\right)}{\left(u_{1}+u\right)^{2}} \mathcal{S}+P\left(u^{2}\right)\left(\frac{1}{\left(u+u_{1}\right)^{2}}+\frac{2}{\left(u+u_{1}\right)\left(u+u_{-1}\right)}-\frac{1}{\left(u+u_{-1}\right)^{2}}\right)+ \\
+P\left(u^{2}\right)^{\prime}\left(\frac{1}{2 u}-\frac{1}{u+u_{1}}\right)+\frac{P\left(u^{2}\right)}{\left(u+u_{-1}\right)^{2}} \mathcal{S}^{-1}+2 u_{t}(\mathcal{S}-1)^{-1} \delta_{u} \rho
\end{gathered}
$$


- Нетривиальная симметрия:

$$
\begin{aligned}
\mathcal{R}\left(u_{t}\right)= & \frac{P\left(u^{2}\right) P\left(u_{1}^{2}\right)}{\left(u+u_{1}\right)^{2}}\left(\frac{1}{u_{1}+u_{2}}-\frac{1}{u_{1}-u_{-1}}\right)- \\
& -\frac{P\left(u^{2}\right) P\left(u_{-1}^{2}\right)}{\left(u+u_{-1}\right)^{2}}\left(\frac{1}{u_{-1}+u_{-2}}-\frac{1}{u_{-1}-u_{1}}\right)+ \\
& +2 P\left(u^{2}\right) \frac{\alpha u_{1}^{2} u_{-1}^{2}+\beta u_{1} u_{-1}+\gamma}{\left(u+u_{1}\right)\left(u_{1}-u_{-1}\right)\left(u+u_{-1}\right)} .
\end{aligned}
$$

- Мастер-симметрия:

$$
\begin{array}{ll}
n u_{t}+\frac{P\left(u^{2}\right)}{u+u_{-1}}-\alpha u^{3}-\beta u & \text { при } \gamma=0, \\
n u_{t}+\frac{P\left(u^{2}\right)}{u+u_{-1}}-\alpha u^{3}-\frac{\beta}{2} u & \text { при } \beta^{2}-4 \alpha \gamma=0, \\
n u_{t}+\frac{P\left(u^{2}\right)}{u+u_{-1}} & \text { при } \alpha=0 .
\end{array}
$$

Здесь мы нашли только мастер-симметрии для некоторых частных случаев. Для приведенного выше гамильтонова оператора имеем $\mathcal{H} \delta_{u} \rho=\mathcal{R}\left(u_{t}\right)$. Кроме того, гамильтонов оператор, симплектический оператор и рекурсионный оператор удовлетворяют соотношению

$$
\mathcal{H} \mathcal{J}=\mathcal{R}^{2}-2 \beta \mathcal{R}+\beta^{2}-4 \alpha \gamma
$$

Цепочка Калоджеро-Дегаспериса [39]

$$
u_{t}=\frac{1}{4}\left(1-u^{2}\right)\left(b^{2}-a^{2} u^{2}\right)\left(\frac{1}{u_{1}+u}-\frac{1}{u+u_{-1}}\right)
$$

представляет собой один из частных случаев уравнения V2. Авторы работы [39] предложили другую мастер-симметрию, введя зависимость коэффициентов $a$ и $b$ от времени мастер-симметрии.

4.4.3. Уравнение V3 (70).

- Гамильтонова структура:

$$
\mathcal{H}=\frac{Q(u)}{u_{1}-u} \mathcal{S} \frac{Q(u)}{u-u_{-1}}-\frac{Q(u)}{u-u_{-1}} \mathcal{S}^{-1} \frac{Q(u)}{u_{1}-u}+u_{t}(\mathcal{S}+1)(\mathcal{S}-1)^{-1} u_{t} .
$$

- Симплектический оператор:

$$
\begin{aligned}
\mathcal{J}=\frac{1}{\left(u_{1}-u\right)^{2}} \mathcal{S}-\mathcal{S}^{-1} \frac{1}{\left(u_{1}-u\right)^{2}}-\delta_{u} \rho(\mathcal{S}+1)(\mathcal{S}-1)^{-1} \delta_{u} \rho- \\
\quad-\left(2 \alpha \gamma-\frac{\beta^{2}}{4}\right) \frac{u^{2}}{Q(u)}(\mathcal{S}-1)(\mathcal{S}+1)^{-1} \frac{u^{2}}{Q(u)}-\beta \delta \frac{u}{Q(u)}(\mathcal{S}-1)(\mathcal{S}+1)^{-1} \frac{u}{Q(u)}- \\
\quad-\left(2 \gamma \epsilon-\frac{\delta^{2}}{4}\right) \frac{1}{Q(u)}(\mathcal{S}-1)(\mathcal{S}+1)^{-1} \frac{1}{Q(u)}+ \\
\quad+(2 \alpha \delta+\beta \gamma)\left(\frac{u^{2}}{Q(u)}(\mathcal{S}+1)^{-1} \frac{u}{Q(u)}-\frac{u}{Q(u)} \mathcal{S}(\mathcal{S}+1)^{-1} \frac{u^{2}}{Q(u)}\right)-
\end{aligned}
$$




$$
\begin{aligned}
& -\left(\frac{\beta \delta}{2}-\gamma^{2}-4 \alpha \epsilon\right)\left(\frac{u^{2}}{Q(u)}(\mathcal{S}+1)^{-1} \frac{1}{Q(u)}-\frac{1}{Q(u)} \mathcal{S}(\mathcal{S}+1)^{-1} \frac{u^{2}}{Q(u)}\right)+ \\
& +(\gamma \delta+2 \beta \epsilon)\left(\frac{u}{Q(u)}(\mathcal{S}+1)^{-1} \frac{1}{Q(u)}-\frac{1}{Q(u)} \mathcal{S}(\mathcal{S}+1)^{-1} \frac{u}{Q(u)}\right)
\end{aligned}
$$

где

$$
\rho=\frac{1}{2} \ln \frac{Q(u)}{\left(u_{1}-u\right)^{2}},
$$

и, таким образом,

$$
\delta_{u} \rho=\frac{1}{u_{1}-u}-\frac{1}{u-u_{-1}}+\frac{Q^{\prime}(u)}{2 Q(u)} .
$$

- Рекурсионный оператор:

$$
\begin{gathered}
\mathcal{R}=\frac{Q(u)}{\left(u_{1}-u\right)^{2}} \mathcal{S}+Q(u)\left(\frac{1}{\left(u-u_{-1}\right)^{2}}+\frac{2}{\left(u_{1}-u\right)\left(u-u_{-1}\right)}-\frac{1}{\left(u_{1}-u\right)^{2}}\right)- \\
-\frac{Q^{\prime}(u)}{u_{1}-u}-2 \alpha u^{2}-\beta u-\gamma+\frac{Q(u)}{\left(u-u_{-1}\right)^{2}} \mathcal{S}^{-1}-2 u_{t}(\mathcal{S}-1)^{-1} \delta_{u} \rho .
\end{gathered}
$$

- Нетривиальная симметрия:

$$
\begin{aligned}
\mathcal{R}\left(u_{t}\right)= & \frac{Q(u) Q\left(u_{1}\right)}{\left(u_{1}-u\right)^{2}\left(u_{2}-u_{1}\right)}+\frac{Q(u) Q\left(u_{-1}\right)}{\left(u-u_{-1}\right)^{2}\left(u_{-1}-u_{-2}\right)}+\alpha Q(u)\left(u_{1}-u_{-1}\right)+ \\
& +Q(u)\left(\frac{Q(u)}{\left(u_{1}-u\right)\left(u-u_{-1}\right)}+2 \alpha u^{2}+\beta u\right)\left(\frac{1}{u_{1}-u}+\frac{1}{u-u_{-1}}\right) .
\end{aligned}
$$

- Мастер-симметрия:

$$
\begin{array}{ll}
n u_{t}-\frac{Q(u)}{u+u_{-1}}+\alpha u^{3}+\beta u^{2}+\gamma u & \text { при } \delta=\epsilon=0, \\
n u_{t}-\frac{Q(u)}{u+u_{-1}} & \text { при } \alpha=\beta=0 .
\end{array}
$$

Как и в случае уравнения V2 (см. п. 4.4.2), мы не смогли найти мастер-симметрию, справедливую для полиномов $Q$ (80) с произвольными коэффициентами. Однако, если искать мастер-симметрию в виде

$$
n u_{t}-\frac{Q(u)}{u+u_{-1}}+\sum_{i=0}^{3} c_{i} u^{i}, \quad c_{i} \in \mathbb{C},
$$

можно определить постоянные $c_{i}$ для некоторых полиномов $Q$. Два примера приведены выше.

Отметим, что $\mathcal{H} \delta_{u} \rho=\mathcal{R}\left(u_{t}\right)$, а произведение гамильтонова оператора на симплектический порождает квадрат рекурсионного оператора, т. е.

$$
\mathcal{H} \mathcal{J}=\mathcal{R}^{2}
$$




\section{5. Цепочка Нариты-Ито-Богоявленского.}

- Уравнение [45], [54], [55]:

$$
u_{t}=u\left(\sum_{k=1}^{p} u_{k}-\sum_{k=1}^{p} u_{-k}\right), \quad p \in \mathbb{N} .
$$

- Гамильтонова структура [15]:

$$
\mathcal{H}=u\left(\sum_{i=1}^{p} \mathcal{S}^{i}-\sum_{i=1}^{p} \mathcal{S}^{-i}\right) u, \quad f=u .
$$

- Рекурсионный оператор [13]:

$$
\mathcal{R}=u\left(\mathcal{S}-\mathcal{S}^{-p}\right)(\mathcal{S}-1)^{-1} \prod_{i=1}^{\rightarrow p}\left(\mathcal{S}^{p+1-i} u-u \mathcal{S}^{-i}\right)\left(\mathcal{S}^{p-i} u-u \mathcal{S}^{-i}\right)^{-1}
$$

где $\prod_{i=1}^{\rightarrow p}$ означает упорядочение значений $i$ от 1 до $p$, т. е. $\prod_{i=1}^{\rightarrow p} a_{i}=a_{1} a_{2} \cdots a_{p}$.

- Нетривиальная симметрия:

$$
\mathcal{R}\left(u_{t}\right)=u\left(1-\mathcal{S}^{-(p+1)}\right) \mathcal{S}^{1-p} \sum_{0 \leqslant i \leqslant j \leqslant 2 p-1} u_{j} u_{i+p} .
$$

- Мастер-симметрия [13]: $\mathcal{R}(u)$.

- Представление Лакса [45]:

$$
L=\mathcal{S}+u \mathcal{S}^{-p}, \quad A=\left(L^{(p+1)}\right)_{\geqslant 0},
$$

где $(\cdot) \geqslant 0$ означает взятие в $L^{(p+1)}$ членов с неотрицательными степенями $\mathcal{S}$.

Несколько симметрий высшего порядка для $p=1,2$ или 3 явно приведены в работе [15], где авторы также изучили их гамильтонов оператор, рекурсионный оператор и мастер-симметрию для $p=1,2$.

Цепочка Нариты-Ито-Богоявленского известна как интегрируемая дискретизация уравнения Кортевега-де Фриза. Она также представима в виде

$$
v_{t}=v\left(\prod_{k=1}^{p} v_{k}-\prod_{k=1}^{p} v_{-k}\right)
$$

который связан с цепочкой Нариты-Ито-Богоявленского с помощью преобразования $u=\prod_{k=0}^{p-1} v_{k}$ при фиксированном $p$.

Взяв $p=1$, получим хорошо известную цепочку Вольтерра из п. 4.1. Таким образом, эти цепочки можно считать обобщением цепочки Вольтерра.

Пусть $u=\prod_{k=0}^{p} w_{k}$. Тогда $w$ удовлетворяет так называемой модифицированной цепочке Богоявленского

$$
w_{t}=w^{2}\left(\prod_{k=1}^{p} w_{k}-\prod_{k=1}^{p} w_{-k}\right)
$$

Приведенный выше рекурсионный оператор для цепочки Нариты-Ито-Богоявленского сильно нелокальный (так же как и мастер-симметрия). Недавно Свинин [56] 
вывел явные формулы, выражающие ее обобщенные симметрии через семейство однородных разностных полиномов. Свойства этих однородных разностных полиномов [57] позволяют нам доказать локальность этого бесконечного ряда симметрий [13].

Семейство интегрируемых дискретных иерархий, связанных с дробными операторами Лакса, было введено Адлером и Постниковым [58], [59]. Одним из простых примеров является уравнение

$$
u_{t}=u^{2}\left(\prod_{k=1}^{p} u_{k}-\prod_{k=1}^{p} u_{-k}\right)-u\left(\prod_{k=1}^{p-1} u_{k}-\prod_{k=1}^{p-1} u_{-k}\right), \quad 2 \leqslant p \in \mathbb{N}
$$

которое представляет собой интегрируемое обобщение уравнения Савады-Котеры. Это уравнение можно считать неоднородным обобщением цепочек Богоявленского. Проблема построения гамильтоновой структуры и рекурсионного оператора для этого семейства уравнений еще не решена.

Классификация эволюционных дифференциально-разностных уравнений высшего порядка даже в простейшем скалярном случае в настоящее время отсутствует.

\section{6. Цепочка Тоды.}

- Уравнение [60]:

$$
q_{t t}=e^{q_{1}-q}-e^{q-q_{-1}} .
$$

В координатах Манакова-Флашки [61], [46], которые вводятся по формулам $u=$ $e^{q_{1}-q}, v=q_{t}$, его можно переписать в виде двухкомпонентной эволюционной системы:

$$
\begin{aligned}
& u_{t}=u\left(v_{1}-v\right), \\
& v_{t}=u-u_{-1} .
\end{aligned}
$$

- Гамильтонова структура [41], [39], [36]:

$$
\begin{array}{rlr}
\mathcal{H}_{1} & =\left(\begin{array}{cc}
0 & u(\mathcal{S}-1) \\
\left(1-\mathcal{S}^{-1}\right) u & 0
\end{array}\right), & f_{1}=u+\frac{v^{2}}{2}, \\
\mathcal{H}_{2}=\left(\begin{array}{cc}
u\left(\mathcal{S}-\mathcal{S}^{-1}\right) u & u(\mathcal{S}-1) v \\
v\left(1-\mathcal{S}^{-1}\right) u & u \mathcal{S}-\mathcal{S}^{-1} u
\end{array}\right), & f_{2}=v .
\end{array}
$$

- Рекурсионный оператор:

$$
\begin{aligned}
\mathcal{R} & =\mathcal{H}_{2} \mathcal{H}_{1}^{-1}=\left(\begin{array}{cc}
v_{1}+u\left(v_{1}-v\right)(\mathcal{S}-1)^{-1} \frac{1}{u} & u \mathcal{S}+u \\
1+\mathcal{S}^{-1}+\left(u-u_{-1}\right)(\mathcal{S}-1)^{-1} \frac{1}{u} & v
\end{array}\right)= \\
& =\left(\begin{array}{cc}
v_{1} & u \mathcal{S}+u \\
1+\mathcal{S}^{-1} & v
\end{array}\right)+\left(\begin{array}{c}
u\left(v_{1}-v\right) \\
u-u_{-1}
\end{array}\right)(\mathcal{S}-1)^{-1}\left(\begin{array}{cc}
\frac{1}{u} & 0
\end{array}\right) .
\end{aligned}
$$

- Нетривиальная симметрия:

$$
\mathcal{R}\left(\begin{array}{c}
u_{t} \\
v_{t}
\end{array}\right)=\left(\begin{array}{c}
u\left(v_{1}^{2}-v^{2}+u_{1}-u_{-1}\right) \\
u\left(v_{1}+v\right)-u_{-1}\left(v_{-1}+v\right)
\end{array}\right) .
$$


- Мастер-симметрия [39]:

$$
\mathcal{R}\left(\begin{array}{l}
u \\
\frac{v}{2}
\end{array}\right)=\left(\begin{array}{c}
n u_{t}+\frac{3}{2} u v_{1}+\frac{1}{2} u v \\
n v_{t}+u+u_{-1}+\frac{v^{2}}{2}
\end{array}\right) .
$$

- Представление Лакса:

$$
M=\left(\begin{array}{cc}
\lambda+v_{1} & u \\
-1 & 0
\end{array}\right), \quad U=\left(\begin{array}{cc}
0 & -u \\
1 & \lambda+v
\end{array}\right)
$$

Нелинейное уравнение Хироты [62]

$$
\begin{aligned}
& u_{t}=v_{1}-v, \\
& v_{t}=v\left(u-u_{-1}\right)
\end{aligned}
$$

связано с цепочкой Тоды (82) простым обратимым преобразованием. А именно, пусть $u=q$ и $v=p_{-1}$. Тогда переменные $p$ и $q$ удовлетворяют уравнению Тоды. Все его свойства можно получить из свойств цепочки Тоды.

\section{7. Релятивистская система Тоды.}

- Уравнение [63]:

$$
q_{t, t}=q_{t} q_{-1, t} \frac{e^{q_{-1}-q}}{1+e^{q_{-1}-q}}-q_{t} q_{1, t} \frac{e^{q-q_{1}}}{1+e^{q-q_{1}}} .
$$

Введем следующие зависимые переменные [64], [65]:

$$
u=\frac{q_{t} e^{q-q_{1}}}{1+e^{q-q_{1}}}, \quad v=\frac{q_{t}}{1+e^{q-q_{1}}} .
$$

Тогда уравнение можно записать в виде

$$
\begin{aligned}
& u_{t}=u\left(u_{-1}-u_{1}+v-v_{1}\right), \\
& v_{t}=v\left(u_{-1}-u\right) .
\end{aligned}
$$

- Гамильтонова структура [65]:

$$
\begin{aligned}
\mathcal{H}_{1}=\left(\begin{array}{cc}
0 & u(1-\mathcal{S}) \\
\left(\mathcal{S}^{-1}-1\right) u & u \mathcal{S}-\mathcal{S}^{-1} u
\end{array}\right), & f_{1}=\frac{1}{2}\left(u^{2}+v^{2}\right)+u v+u_{1} u+u v_{1}, \\
\mathcal{H}_{2}=\left(\begin{array}{cc}
u\left(\mathcal{S}^{-1}-\mathcal{S}\right) u & u(1-\mathcal{S}) v \\
v\left(\mathcal{S}^{-1}-1\right) u & 0
\end{array}\right), & f_{2}=u+v .
\end{aligned}
$$

- Рекурсионный оператор [65]:

$$
\begin{aligned}
\mathcal{R} & =\mathcal{H}_{2} \mathcal{H}_{1}^{-1}= \\
& =\left(\begin{array}{cc}
u \mathcal{S}+u+v_{1}+u_{1}+u \mathcal{S}^{-1}-u\left(v-v_{1}+u_{-1}-u_{1}\right)(\mathcal{S}-1)^{-1} \frac{1}{u} & u \mathcal{S}+u \\
v+v \mathcal{S}^{-1}-v\left(u_{-1}-u\right)(\mathcal{S}-1)^{-1} \frac{1}{u} & v
\end{array}\right)= \\
& =\left(\begin{array}{cc}
u \mathcal{S}+u+v_{1}+u_{1}+u \mathcal{S}^{-1} & u \mathcal{S}+u \\
v+v \mathcal{S}^{-1} & v
\end{array}\right)-\left(\begin{array}{c}
u_{t} \\
v_{t}
\end{array}\right)(\mathcal{S}-1)^{-1}\left(\begin{array}{cc}
\frac{1}{u} & 0
\end{array}\right) .
\end{aligned}
$$


- Нетривиальная симметрия:

$$
\left(\begin{array}{c}
u u_{-1}\left(u+u_{-1}+u_{-2}+2 v+v_{-1}\right)- \\
-u u_{1}\left(u_{2}+u_{1}+u+2 v_{1}+v_{2}\right)+u^{2}\left(v-v_{1}\right)+u\left(v^{2}-v_{1}^{2}\right) \\
v u_{-1}\left(u_{-2}+u_{-1}+v+v_{-1}\right)-u v\left(u_{1}+u+v_{1}+v\right)
\end{array}\right)
$$

- Мастер-симметрия [65]:

$$
\mathcal{R}\left(\begin{array}{l}
u \\
v
\end{array}\right)=\left(\begin{array}{c}
-n u_{t}+u\left(v+2 v_{1}+u+2 u_{1}+u_{-1}\right) \\
-n v_{t}+v\left(u+v+u_{-1}\right)
\end{array}\right), \quad(\mathcal{S}-1)^{-1} 1=n .
$$

- Представление Лакса:

$$
M=\left(\begin{array}{cc}
\lambda v-\lambda^{-1} & u_{-1} \\
-1 & 0
\end{array}\right), \quad U=\left(\begin{array}{cc}
-\lambda^{-2}-u_{-1} & \lambda^{-1} u_{-1} \\
-\lambda^{-1} & -u_{-2}-v_{-1}
\end{array}\right) .
$$

Как отмечено в работе [65], оператор, обратный к этому рекурсионному оператору $\mathcal{R}$, тоже слабо нелокальный:

$$
\begin{aligned}
\mathcal{R}^{-1}=\mathcal{H}_{1} \mathcal{H}_{2}^{-1}= & \left(\begin{array}{cc}
\frac{1}{v_{1}} & -\frac{u}{v_{1}^{2}} \mathcal{S}+\frac{u}{v^{2}}-\frac{2 u}{v v_{1}} \\
-\mathcal{S}^{-1} \frac{1}{v}-\frac{1}{v_{1}} & \frac{u}{v_{1}^{2}} \mathcal{S}+\mathcal{S}^{-1} \frac{u}{v^{2}}+\frac{2 u}{v v_{1}}+\frac{1}{v}
\end{array}\right)+ \\
+ & \left(\begin{array}{c}
\frac{u}{v_{1}}-\frac{u}{v} \\
\frac{u-1}{v_{-1}}-\frac{u}{v_{1}}
\end{array}\right)(\mathcal{S}-1)^{-1}\left(\begin{array}{cc}
\frac{1}{u} & -\frac{2}{v}
\end{array}\right)
\end{aligned}
$$

Однако иерархии, связанные с рекурсионными операторами $\mathcal{R}$ и $\mathcal{R}^{-1}$, имеют различные начальные (затравочные) симметрии. Что касается оператора $\mathcal{R}$, то его затравкой является правая часть уравнения (83), а затравкой для оператора $\mathcal{R}^{-1}$ является

$$
\sigma=\left(\begin{array}{c}
\frac{u}{v_{1}}-\frac{u}{v} \\
\frac{u_{-1}}{v_{-1}}-\frac{u}{v_{1}}
\end{array}\right)
$$

Более того, оператор $\mathcal{R}$, действующий на $\sigma$, и оператор $\mathcal{R}^{-1}$, действующий на правую часть уравнения, не порождают новые симметрии.

Между потоком, соответствующим $\sigma$, и уравнением (83) существует преобразование Миуры $u=-u_{-1}^{\prime} / v^{\prime} v_{-1}^{\prime}, v=-1 / v_{-1}^{\prime}$, где $u^{\prime}$ и $v^{\prime}$ означают зависимые переменные для $\sigma$.

В работе [64] изучаются другие интегрируемые уравнения, связанные с релятивистской цепочкой Тоды. Например, уравнение

$$
q_{t, t}=q_{-1, t} e^{q_{-1}-q}-e^{2 q_{-1}-2 q}-q_{1, t} e^{q-q_{1}}+e^{2 q-2 q_{1}}
$$

можно записать также в виде системы (83), полагая

$$
u=e^{q-q_{1}}, \quad v=q_{t}-e^{q_{-1}-q}-e^{q-q_{1}} .
$$

3 Теоретическая и математическая физика, т. 177, № 3, 2013 г. 


\section{8. Двухкомпонентная цепочка Вольтерра.}

- Уравнение [41]:

$$
\begin{aligned}
& u_{t}=u\left(v_{1}-v\right), \\
& v_{t}=v\left(u-u_{-1}\right)
\end{aligned}
$$

- Гамильтонова структура [41]:

$$
\begin{aligned}
\mathcal{H}_{1}=\left(\begin{array}{cc}
0 & u(\mathcal{S}-1) v \\
v\left(1-\mathcal{S}^{-1}\right) u & 0
\end{array}\right), & f_{1}=u+v \\
\mathcal{H}_{2}=\left(\begin{array}{cc}
u\left(\mathcal{S} v-v \mathcal{S}^{-1}\right) u & u(u \mathcal{S}-u+\mathcal{S} v-v) v \\
v\left(u-\mathcal{S}^{-1} u+v-v \mathcal{S}^{-1}\right) u & v\left(u \mathcal{S}-\mathcal{S}^{-1} u\right) v
\end{array}\right), & f_{2}=\ln u
\end{aligned}
$$

- Рекурсионный оператор:

$$
\begin{aligned}
\mathcal{R} & =\mathcal{H}_{2} \mathcal{H}_{1}^{-1}= \\
& =\left(\begin{array}{cc}
u+v_{1}+u\left(v_{1}-v\right)(\mathcal{S}-1)^{-1} \frac{1}{u} & u \mathcal{S}+\frac{u v_{1}}{v}+u\left(v_{1}-v\right)(\mathcal{S}-1)^{-1} \frac{1}{v} \\
v+v \mathcal{S}^{-1}+v\left(u-u_{-1}\right)(\mathcal{S}-1)^{-1} \frac{1}{u} & u+v+v\left(u-u_{-1}\right)(\mathcal{S}-1)^{-1} \frac{1}{v}
\end{array}\right)= \\
& =\left(\begin{array}{cc}
u+v_{1} & u \mathcal{S}+\frac{u v_{1}}{v} \\
v+v \mathcal{S}^{-1} & u+v
\end{array}\right)+\left(\begin{array}{c}
u\left(v_{1}-v\right) \\
v\left(u-u_{-1}\right)
\end{array}\right)(\mathcal{S}-1)^{-1}\left(\begin{array}{cc}
\frac{1}{u} & \frac{1}{v}
\end{array}\right) .
\end{aligned}
$$

- Нетривиальная симметрия:

$$
\mathcal{R}\left(\begin{array}{l}
u_{t} \\
v_{t}
\end{array}\right)=\left(\begin{array}{c}
u^{2}\left(v_{1}-v\right)+u\left(v_{1}^{2}-v^{2}+v_{1} u_{1}-v u_{-1}\right) \\
v^{2}\left(u-u_{-1}\right)+v\left(u^{2}-u_{-1}^{2}+u v_{1}-u_{-1} v_{-1}\right)
\end{array}\right) .
$$

- Мастер-симметрия [39]:

$$
\mathcal{R}\left(\begin{array}{l}
u \\
v
\end{array}\right)=\left(\begin{array}{c}
2 n u_{t}+u^{2}+3 u v_{1} \\
2 n v_{t}+v u_{-1}+2 u v+v^{2}
\end{array}\right)
$$

- Представление Лакса [66]:

$$
L=\lambda \mathcal{S}^{-1}+v+u_{-1}+\lambda^{-1} u v \mathcal{S}, \quad A=\lambda^{-1} u v \mathcal{S}
$$

Эта система происходит из цепочки Вольтерра из п. 4.1, переписанной через переменную $w$, т. е.

$$
w_{t}=w\left(w_{1}-w_{-1}\right)
$$


с помощью переобозначений $u(n, t)=w(2 n, t)$ и $v(n, t)=w(2 n-1, t)$. Это уравнение связано с уравнением Тоды (82), записанным в переменных $\bar{u}$ и $\bar{v}$, с помощью преобразования Миуры [67]

$$
\bar{u}=u v, \quad \bar{v}=u_{-1}+v .
$$

Фактически (мастер-)симметрии, законы сохранения и локальные гамильтоновы структуры этой системы легко получить из цепочки Вольтерра. Например, первый гамильтонов оператор $\mathcal{H}_{1}$ выводится следующим образом. Симметрии цепочки Вольтерра (85) имеют вид

$$
w_{\tau}=w\left(\mathcal{S}-\mathcal{S}^{-1}\right) w Q[n]=w w_{1} Q[n+1]-w w_{-1} Q[n-1],
$$

где $Q[n]$ - вариационная производная сохраняющейся плотности для цепочки (85). Теперь выпишем четную и нечетную цепочки и переименуем их, вводя переменные $u$ и $v$ соответственно. Получим

$$
u_{\tau}=u v_{1} Q[2 n+1]-u v Q[2 n-1], \quad v_{\tau}=v u Q[2 n]-v u_{-1} Q[2 n-2],
$$

T. e.

$$
\left(\begin{array}{l}
u_{\tau} \\
v_{\tau}
\end{array}\right)=\left(\begin{array}{cc}
0 & u(\mathcal{S}-1) v \\
v\left(1-\mathcal{S}^{-1}\right) u & 0
\end{array}\right)\left(\begin{array}{c}
Q[2 n] \\
Q[2 n-1]
\end{array}\right) .
$$

Используя тот же метод, можно вывести гамильтониан $\mathcal{H}_{2}$, указанный в списке. Построение подобного рода верно для всех скалярных уравнений. В случае нелокального оператора оно более сложно.

\section{9. Релятивистская цепочка Вольтерра.}

- Уравнение [66], [67], [28]:

$$
\begin{aligned}
& u_{t}=u\left(v-v_{-1}+u v-u_{-1} v_{-1}\right), \\
& v_{t}=v\left(u_{1}-u+u_{1} v_{1}-u v\right) .
\end{aligned}
$$

- Гамильтонова структура [66]:

$\mathcal{H}_{1}=\left(\begin{array}{cc}0 & u\left(1-\mathcal{S}^{-1}\right) v \\ v(\mathcal{S}-1) u & 0\end{array}\right), \quad f_{1}=u+v+u v$,

$\mathcal{H}_{2}=\left(\begin{array}{cc}u v(1+u) \mathcal{S} u-u \mathcal{S}^{-1} u v(1+u) & u v(u+v+u v)- \\ v(\mathcal{S} u v \mathcal{S}+v \mathcal{S}+\mathcal{S} u) u-u v(u+v+u v) & -u\left(\mathcal{S}^{-1} u v \mathcal{S}^{-1}+u \mathcal{S}^{-1}+\mathcal{S}^{-1} v\right) v \\ \mathcal{S} u v(1+v)-u v(1+v) \mathcal{S}^{-1} v\end{array}\right)$,

$f_{2}=\ln u$ или $f_{2}=\ln v$.

- Рекурсионный оператор:

$$
\begin{aligned}
\mathcal{R}=\mathcal{H}_{2} \mathcal{H}_{1}^{-1}=\left(\begin{array}{cc}
u v_{-1} \mathcal{S}^{-1}+u+v+u v & u\left(1+u_{-1}\right) \mathcal{S}^{-1}+u(1+u) \\
v\left(1+v_{1}\right) \mathcal{S}+\frac{u_{1} v\left(1+v_{1}\right)}{u} & u_{1} v \mathcal{S}+u_{1}+v+u_{1} v_{1}
\end{array}\right)+ \\
+\left(\begin{array}{cc}
u\left(v-v_{-1}+u v-u_{-1} v_{-1}\right) \\
v\left(u_{1}-u+u_{1} v_{1}-u v\right)
\end{array}\right)(\mathcal{S}-1)^{-1}\left(\begin{array}{cc}
\frac{1}{u} & \frac{1}{v}
\end{array}\right) .
\end{aligned}
$$


- Нетривиальная симметрия:

$$
\mathcal{R}\left(\begin{array}{c}
u_{t} \\
v_{t}
\end{array}\right)=\left(\begin{array}{c}
u v(1+u)\left(u+u_{1}+u_{1} v_{1}\right)+u v^{2}(1+u)^{2}-u v_{-1}^{2}\left(1+u_{-1}\right)^{2}-u^{2} v_{-1}- \\
-u_{-1} u v_{-1}\left(1+u+v_{-2}+u_{-1}+u_{-2} v_{-2}\right) \\
u_{1} v v_{1}\left(1+2 u_{1}+u_{2} v_{2}+u_{2}\right)+u_{1}^{2} v+u_{1} v v_{1}^{2}\left(1+u_{1}\right)+v^{2} u_{1}\left(1+v_{1}\right)- \\
-u v(1+v)\left(v+v_{-1}+u_{-1} v_{-1}\right)-u^{2} v(1+v)^{2}
\end{array}\right) .
$$

- Представление Лакса:

$$
\begin{gathered}
M=\left(\begin{array}{cc}
\lambda^{2}+2 u_{1}+2 v+1 & -\lambda(2 v+1)-\lambda^{-1}\left(2 u_{1}+1\right) \\
-\lambda\left(2 u_{1}+1\right)-\lambda^{-1}(2 v+1) & \lambda^{-2}+2 u_{1}+2 v+1
\end{array}\right), \\
U=\left(\begin{array}{cc}
-\frac{\lambda^{2}-\lambda^{-2}}{8}+u v+\frac{u}{2}+\frac{v}{2} & \lambda \frac{(2 v+1)}{4}+\lambda^{-1} \frac{(2 u+1)}{4} \\
\lambda \frac{(2 u+1)}{4}+\lambda^{-1} \frac{(2 v+1)}{4} & \frac{\lambda^{2}-\lambda^{-2}}{8}+u v+\frac{u}{2}+\frac{v}{2}
\end{array}\right) .
\end{gathered}
$$

Представление Лакса, полученное в работе [66], имеет вид

$$
U_{t}=U C-A U, \quad W_{t}=W B-C W, \quad V_{t}=V B-A V,
$$

где разностные операторы $U, V, W, A, B$ и $C$ заданы выражениями

$$
\begin{aligned}
U & =u+\lambda \mathcal{S}^{-1}, & & A=u+u_{-1} v_{-1}+v_{-1}+\lambda \mathcal{S}^{-1}, \\
W & =1+\lambda^{-1} v \mathcal{S}, & & B=u+u v+v_{-1}+\lambda \mathcal{S}^{-1}, \\
V & =1-\lambda^{-1} u v \mathcal{S}, & & C=u+u v+v+\lambda \mathcal{S}^{-1} .
\end{aligned}
$$

У рекурсионного оператора $\mathcal{R}$ есть слабо нелокальный обратный оператор:

$$
\begin{aligned}
& \mathcal{R}^{\prime}=\left(\begin{array}{cc}
\frac{u v}{\left(u_{1}+v+1\right)^{2}} \mathcal{S}+ & -\frac{u(u+1)}{\left(u+v_{-1}+1\right)^{2}} \mathcal{S}^{-1}- \\
+\frac{u v(u+1)+\left(\left(1+v_{-1}\right)^{2}+u\right)\left(u_{1}+1\right)}{\left(u+v_{-1}+1\right)^{2}\left(u_{1}+v+1\right)} & -\frac{u\left(u_{1}+1\right)}{\left(u_{1}+v+1\right)^{2}} \\
-\frac{v(1+v)}{\left(u_{1}+v+1\right)^{2}} \mathcal{S}- & \frac{u v}{\left(u+v_{-1}+1\right)^{2}} \mathcal{S}^{-1}+ \\
-\frac{v\left(u^{2}+u^{2} v+2 u_{1} v_{-1}+u_{1}+u_{1} v_{-1}^{2}\right)}{u\left(u+v_{-1}+1\right)\left(u_{1}+v+1\right)} & +\frac{\left(1+u_{1}\right)(1+v)}{\left(u_{1}+v+1\right)^{2}}
\end{array}\right)+ \\
& +\left(\begin{array}{c}
-\frac{u v_{-1}}{u+v_{-1}+1}+\frac{u v}{u_{1}+v+1} \\
-\frac{u v}{u+v_{-1}+1}+\frac{u_{1} v}{u_{1}+v+1}
\end{array}\right)(\mathcal{S}-1)^{-1}\left(\frac{2}{u+v_{-1}+1}-\frac{1}{u} \frac{2}{u_{1}+v+1}-\frac{1}{v}\right) .
\end{aligned}
$$

Действительно, он коммутирует с $\mathcal{R}$ и удовлетворяет соотношению $\mathcal{R}^{\prime}(\mathcal{R}+\mathrm{id})=\mathrm{id}$. Он связан с релятивистским уравнением Тоды (83), записанным в переменных $\bar{u}$ и $\bar{v}$, с помощью преобразования Миуры $\bar{u}=-u v$ и $\bar{v}=-\left(u+v_{-1}+1\right)$ [67]. Это преобразование похоже на преобразование (86), чем объясняется название этого уравнения. 


\subsection{0. Цепочка Мерола-Рагниско-Ту.}

- Уравнение [15], [68]:

$$
\begin{aligned}
& u_{t}=u_{1}-u^{2} v, \\
& v_{t}=-v_{-1}+v^{2} u .
\end{aligned}
$$

- Гамильтонова структура [15]:

$$
\mathcal{H}=\left(\begin{array}{cc}
0 & 1 \\
-1 & 0
\end{array}\right), \quad f=u_{1} v-\frac{u^{2} v^{2}}{2} .
$$

- Рекурсионный оператор [15]:

$$
\mathcal{R}=\left(\begin{array}{cc}
\mathcal{S}-2 u v & -u^{2} \\
v^{2} & \mathcal{S}^{-1}
\end{array}\right)+2\left(\begin{array}{c}
-u \\
v
\end{array}\right)(\mathcal{S}-1)^{-1}\left(\begin{array}{ll}
v & u
\end{array}\right) .
$$

- Нетривиальная симметрия [15]:

$$
\mathcal{R}\left(\begin{array}{l}
u_{t} \\
v_{t}
\end{array}\right)=\left(\begin{array}{c}
u_{2}-u_{1}^{2} v_{1}-u^{2} v_{-1}-2 u v u_{1}+u^{3} v^{2} \\
-v_{-2}+v_{-1}^{2} u_{-1}+v^{2} u_{1}+2 u v v_{-1}-u^{2} v^{3}
\end{array}\right) .
$$

- Мастер-симметрия [15]:

$$
\mathcal{R}\left(\begin{array}{c}
(n+1) u \\
-n v
\end{array}\right)=\left(\begin{array}{c}
n u_{t}+2 u_{1}-2 u^{2} v-2 u(\mathcal{S}-1)^{-1} u v \\
n v_{t}+v_{-1}+u v^{2}+2 v(\mathcal{S}-1)^{-1} u v
\end{array}\right) .
$$

- Представление Лакса:

$$
M=\left(\begin{array}{cc}
-1 & v \\
u & -2 \lambda-u v
\end{array}\right), \quad U=\left(\begin{array}{cc}
-\lambda & -v_{-1} \\
-u & \lambda
\end{array}\right) .
$$

Рекурсионный оператор $\mathcal{R}$ имеет слабо нелокальный обратный оператор:

$$
\begin{aligned}
& \mathcal{R}^{-1}=\left(\begin{array}{cc}
\frac{1}{\left(u_{-1} v+1\right)^{2}} \mathcal{S}^{-1} & \frac{u_{-1}^{2}}{\left(u_{-1} v+1\right)^{2}} \\
-\frac{v_{1}^{2}}{\left(u v_{1}+1\right)^{2}} & \frac{1}{\left(u v_{1}+1\right)^{2}} \mathcal{S}-\frac{2 u_{-1} v_{1}}{\left(u_{-1} v+1\right)\left(u v_{1}+1\right)}
\end{array}\right)+ \\
& +2\left(\begin{array}{c}
\frac{u_{-1}}{u_{-1} v+1} \\
-\frac{v_{1}}{u v_{1}+1}
\end{array}\right)(\mathcal{S}-1)^{-1}\left(\begin{array}{ll}
\frac{v_{1}}{u v_{1}+1} & \frac{u_{-1}}{u_{-1} v+1}
\end{array}\right) .
\end{aligned}
$$

Симметрия $(u,-v)^{\mathrm{T}}$ является затравочной для $\mathcal{R}$ и $\mathcal{R}^{-1}$.

С помощью обратимого преобразования $t \mapsto-t, u \mapsto-u$ и $v \mapsto v_{1}$ эта цепочка преобразуется в систему

$$
\begin{aligned}
& u_{t}=-u_{1}-u^{2} v_{1}, \\
& v_{t}=v_{-1}+v^{2} u_{-1},
\end{aligned}
$$

которая связана с нелинейной системой Шредингера $u_{t}=u_{x x}+2 u^{2} v,-v_{t}=v_{x x}+$ $2 v^{2} u$, представленной в работе [69]. 


\subsection{1. Цепочка Каупа.}

- Уравнение [69]:

$$
\begin{aligned}
& u_{t}=(u+v)\left(u_{1}-u\right), \\
& v_{t}=(u+v)\left(v-v_{-1}\right) .
\end{aligned}
$$

- Гамильтонова структура [69]:

$$
\mathcal{H}=\left(\begin{array}{cc}
0 & u+v \\
-(u+v) & 0
\end{array}\right), \quad f=u_{1} v-u v
$$

- Рекурсионный оператор:

$$
\begin{aligned}
& \mathcal{R}=\left(\begin{array}{cc}
(u+v) \mathcal{S}+u_{1}-u & 0 \\
0 & (u+v) \mathcal{S}^{-1}+u_{1}-u
\end{array}\right)+\left(\begin{array}{l}
u_{t} \\
v_{t}
\end{array}\right)(\mathcal{S}-1)^{-1}\left(\begin{array}{cc}
\frac{1}{u+v} & \frac{1}{u+v}
\end{array}\right)+ \\
& +\left(\begin{array}{c}
1 \\
-1
\end{array}\right) \mathcal{S}(\mathcal{S}-1)^{-1}\left(v_{-1}-v \quad u_{1}-u\right) .
\end{aligned}
$$

- Нетривиальная симметрия:

$$
\mathcal{R}\left(\begin{array}{l}
u_{t} \\
v_{t}
\end{array}\right)=\left(\begin{array}{c}
(u+v)\left(u u_{1}+u v_{-1}+u_{1} v_{1}-u_{1} u_{2}-u_{2} v_{1}-u_{1} v_{-1}\right) \\
(u+v)\left(u_{-1} v_{-2}+v_{-2} v_{-1}-u_{1} v-u_{-1} v_{-1}+u_{1} v_{-1}-v_{-1} v\right)
\end{array}\right)
$$

- Представление Лакса [69]:

$$
M=\left(\begin{array}{cc}
u-\lambda & u v+\lambda(u+v)+\lambda^{2} \\
1 & v-\lambda
\end{array}\right), \quad U=\left(\begin{array}{cc}
u & (u+\lambda)\left(v_{-1}+\lambda\right) \\
1 & v_{-1}
\end{array}\right) .
$$

Обратный слабо нелокальный рекурсионный оператор имеет вид

$$
\begin{aligned}
\mathcal{R}^{\prime}= & \left(\begin{array}{cc}
\frac{u+v}{(u-1+v)^{2}} \mathcal{S}^{-1} & -\frac{\left(u-u_{-1}\right)}{\left(u_{-1}+v\right)^{2}} \\
-\frac{\left(v_{1}-v\right)}{\left(u+v_{1}\right)^{2}} & \frac{u+v}{\left(u+v_{1}\right)^{2}} \mathcal{S}+\frac{u-u_{-1}-v_{1}+v}{\left(u_{-1}+v\right)\left(u+v_{1}\right)}
\end{array}\right)- \\
& -\left(\begin{array}{c}
1 \\
-1
\end{array}\right)(\mathcal{S}-1)^{-1}\left(\frac{1}{u+v_{1}}-\frac{1}{u+v} \frac{1}{u_{-1}+v}-\frac{1}{u+v}\right)- \\
& -\left(\begin{array}{c}
\frac{u-u_{-1}}{u_{-1}+v} \\
\frac{v_{1}-v}{u+v_{1}}
\end{array}\right)(\mathcal{S}-1)^{-1}\left(\frac{2}{u+v_{1}}-\frac{1}{u+v} \quad \frac{2}{u_{-1}+v}-\frac{1}{u+v}\right)
\end{aligned}
$$

Симметрия $(1,-1)^{\mathrm{T}}$ является затравочной для $\mathcal{R}$ и $\mathcal{R}^{\prime}$. 


\subsection{2. Цепочка Абловица-Ладека.}

- Уравнение [70]:

$$
\left.\begin{array}{c}
u_{t}=(1-u v)\left(\alpha u_{1}-\beta u_{-1}\right) \\
v_{t}=(1-u v)\left(\beta v_{1}-\alpha v_{-1}\right)
\end{array}\right\}:=\alpha K_{1}+\beta K_{-1} .
$$

- Гамильтонова структура [15]:

$$
\mathcal{H}=\left(\begin{array}{cc}
0 & 1-u v \\
-(1-u v) & 0
\end{array}\right), \quad f=\left(\alpha u_{1}-\beta u_{-1}\right) v .
$$

- Рекурсионный оператор [15], [71], [72]:

$$
\begin{aligned}
\mathcal{R}= & \left(\begin{array}{cc}
(1-u v) \mathcal{S}-u_{1} v-u v_{-1} & -u u_{1} \\
v v_{-1} & (1-u v) \mathcal{S}^{-1}
\end{array}\right)+\left(\begin{array}{c}
-u \\
v
\end{array}\right)(\mathcal{S}-1)^{-1}\left(\begin{array}{ll}
v_{-1} & u_{1}
\end{array}\right)- \\
& -\left(\begin{array}{c}
(1-u v) u_{1} \\
-(1-u v) v_{-1}
\end{array}\right)(\mathcal{S}-1)^{-1}\left(\begin{array}{ll}
\frac{v}{1-u v} & \frac{u}{1-u v}
\end{array}\right) .
\end{aligned}
$$

- Нетривиальная симметрия [15]:

$$
\mathcal{R}\left(\begin{array}{c}
(1-u v) u_{1} \\
-(1-u v) v_{-1}
\end{array}\right)=\left(\begin{array}{c}
(1-u v)\left(\left(1-u_{1} v_{1}\right) u_{2}-v u_{1}^{2}-u u_{1} v_{-1}\right) \\
(1-u v)\left(-\left(1-u_{-1} v_{-1}\right) v_{-2}+u v_{-1}^{2}+u_{1} v_{-1} v\right)
\end{array}\right) .
$$

- Мастер-симметрия [15]:

$$
\mathcal{R}\left(\begin{array}{c}
n u \\
-n v
\end{array}\right)=\left(\begin{array}{c}
(n+1)(1-u v) u_{1}-u^{2} v_{-1}-u(\mathcal{S}-1)^{-1} u v_{-1} \\
(1-n)(1-u v) v_{-1}+u v v_{-1}+v(\mathcal{S}-1)^{-1} u v_{-1}
\end{array}\right) .
$$

- Представление Лакса:

$$
M=\left(\begin{array}{cc}
\lambda & u \\
v & \frac{1}{\lambda}
\end{array}\right), \quad U=\alpha\left(\begin{array}{cc}
\lambda^{2}-u v_{-1} & \lambda u \\
\lambda v_{-1} & 0
\end{array}\right)+\beta\left(\begin{array}{cc}
0 & \lambda^{-1} u_{-1} \\
\lambda^{-1} v & \lambda^{-2}-u_{-1} v
\end{array}\right) .
$$

Коэффициенты перед $\alpha$ и $\beta$, а именно $K_{1}$ и $K_{-1}$, являются коммутирующими симметриями для уравнения. Оператор, обратный к данному рекурсионному оператору $\mathcal{R}$, имеет слабо нелокальную форму:

$$
\begin{aligned}
\mathcal{R}^{-1}= & \left(\begin{array}{cc}
(1-u v) \mathcal{S}^{-1} & u u_{-1} \\
-v v_{1} & (1-u v) \mathcal{S}-u v_{1}-u_{-1} v
\end{array}\right)+\left(\begin{array}{c}
u \\
-v
\end{array}\right)(\mathcal{S}-1)^{-1}\left(\begin{array}{ll}
v_{1} & u_{-1}
\end{array}\right)+ \\
& +\left(\begin{array}{cc}
(1-u v) u_{-1} \\
-(1-u v) v_{1}
\end{array}\right)(\mathcal{S}-1)^{-1}\left(\begin{array}{cc}
\frac{v}{1-u v} & \frac{u}{1-u v}
\end{array}\right) .
\end{aligned}
$$

Операторы $\mathcal{R}$ и $\mathcal{R}^{-1}$ имеют общую затравку $\sigma=\left(\begin{array}{c}u \\ -v\end{array}\right)$. Начав с нее, можно генерировать коммутирующие симметрии $\mathcal{R}^{-i}(\sigma)$ и $\mathcal{R}^{i}(\sigma)$ для $i \in \mathbb{N}$. Более подробное изложение структуры алгебры Ли для симметрий и мастер-симметрий можно найти в работе [15]. 


\subsection{3. Цепочка Бруски-Рагниско.}

- Уравнение [73], [66], [41]:

$$
\begin{aligned}
& u_{t}=u_{1} v-u v_{-1}, \\
& v_{t}=v\left(v-v_{-1}\right)
\end{aligned}
$$

- Гамильтонова структура [73], [15]:

$$
\begin{aligned}
\mathcal{H}_{1} & =\left(\begin{array}{cc}
0 & \left(1-\mathcal{S}^{-1}\right) v \\
v(\mathcal{S}-1) & 0
\end{array}\right), & f_{1}=u_{1} v, \\
\mathcal{H}_{2} & =\left(\begin{array}{cc}
v \mathcal{S} u-u \mathcal{S}^{-1} v & v(\mathcal{S}-1) v \\
v\left(1-\mathcal{S}^{-1}\right) v & 0
\end{array}\right), & f_{2}=u .
\end{aligned}
$$

- Рекурсионный оператор [41], [15]:

$$
\begin{aligned}
\mathcal{R} & =\mathcal{H}_{2} \mathcal{H}_{1}^{-1}=\left(\begin{array}{cc}
v \mathcal{S} & u_{1}+u \mathcal{S}^{-1}+\left(u_{1} v-u v_{-1}\right)(\mathcal{S}-1)^{-1} \frac{1}{v} \\
0 & v \mathcal{S}^{-1}+v\left(v-v_{-1}\right)(\mathcal{S}-1)^{-1} \frac{1}{v}
\end{array}\right)= \\
& =\left(\begin{array}{cc}
v \mathcal{S} & u_{1}+u \mathcal{S}^{-1} \\
0 & v \mathcal{S}^{-1}
\end{array}\right)+\left(\begin{array}{c}
u_{t} \\
v_{t}
\end{array}\right)(\mathcal{S}-1)^{-1}\left(\begin{array}{ll}
0 & \frac{1}{v}
\end{array}\right) .
\end{aligned}
$$

- Нетривиальная симметрия [15]:

$$
\mathcal{R}\left(\begin{array}{l}
u_{t} \\
v_{t}
\end{array}\right)=\left(\begin{array}{c}
v v_{1} u_{2}-v_{-2} v_{-1} u \\
v\left(v v_{-1}-v_{-1} v_{-2}\right)
\end{array}\right)
$$

- Мастер-симметрия [15]:

$$
\mathcal{R}\left(\begin{array}{l}
u \\
v
\end{array}\right)=\left(\begin{array}{c}
n u_{t}+2 u_{1} v+u_{-1} u \\
n v_{t}+v v_{-1}
\end{array}\right) .
$$

Рекурсионный оператор $\mathcal{R}$ обладает слабо нелокальным обратным оператором:

$$
\begin{gathered}
\mathcal{R}^{-1}=\mathcal{H}_{1} \mathcal{H}_{2}^{-1}=\left(\begin{array}{cc}
\mathcal{S}^{-1} \frac{1}{v} & -\mathcal{S}^{-1} \frac{u}{v^{2}}-\frac{u}{v^{2}}+\left(\frac{u_{-1}}{v_{-1}}-\frac{u}{v}\right)(\mathcal{S}-1)^{-1} \frac{1}{v} \\
0 & v \mathcal{S} \frac{1}{v^{2}}-\frac{1}{v}+\frac{1}{v_{1}}+\left(\frac{v}{v_{1}}-1\right)(\mathcal{S}-1)^{-1} \frac{1}{v}
\end{array}\right)= \\
=\left(\begin{array}{cc}
\mathcal{S}^{-1} \frac{1}{v} & -\mathcal{S}^{-1} \frac{u}{v^{2}}-\frac{u}{v^{2}} \\
0 & v \mathcal{S} \frac{1}{v^{2}}-\frac{1}{v}+\frac{1}{v_{1}}
\end{array}\right)+\left(\begin{array}{c}
\frac{u_{-1}}{v_{-1}}-\frac{u}{v} \\
\frac{v}{v_{1}}-1
\end{array}\right)(\mathcal{S}-1)^{-1}\left(\begin{array}{ll}
0 & \frac{1}{v}
\end{array}\right) .
\end{gathered}
$$


В этом случае рекурсионные операторы $\mathcal{R}$ и $\mathcal{R}^{-1}$ имеют разные затравки, подобно релятивистской системе Тоды в п. 4.7. Исходной точкой для оператора $\mathcal{R}$ является само уравнение, а затравкой для $\mathcal{R}^{-1}$ является

$$
\sigma=\left(\begin{array}{c}
\frac{u_{-1}}{v_{-1}}-\frac{u}{v} \\
\frac{v}{v_{1}}-1
\end{array}\right)
$$

Операторы $\mathcal{R}^{-1}$, действующий на само уравнение, и $\mathcal{R}$, действующий на $\sigma$, не порождают новых симметрий. Более подробную информацию о структуре алгебры Ли для симметрий и мастер-симметрий можно найти в работе [15].

По существу, цепочка Бруски-Рагниско может быть сведена к линейной, так же как и ее симметрийные потоки. Уравнение для второй компоненты $v_{t}$ не зависит от первой компоненты $u$. Кроме того, скалярные цепочки $v_{t}=v\left(v-v_{-1}\right)$ и $v_{\tau}=$ $v / v_{1}-1$ можно линеаризовать к виду $w_{t}=w_{-1}$ и $w_{t}=w_{1}$ соответственно с помощью преобразования $v=-w_{-1} / w$. Решив его, мы получим линейное уравнение для $u$.

\subsection{4. Цепочка Каупа-Ньюэлла.}

- Уравнение [74]:

$$
\left.\begin{array}{c}
u_{t}=a\left(\frac{u_{1}}{1-u_{1} v_{1}}-\frac{u}{1-u v}\right)+b\left(\frac{u}{1+u v_{1}}-\frac{u_{-1}}{1+u_{-1} v}\right) \\
v_{t}=a\left(\frac{v}{1-u v}-\frac{v_{-1}}{1-u_{-1} v_{-1}}\right)+b\left(\frac{v_{1}}{1+u v_{1}}-\frac{v}{1+u_{-1} v}\right)
\end{array}\right\}:=a K_{1}+b K_{-1} .
$$

- Гамильтонова структура [74]:

$$
\mathcal{H}=\left(\begin{array}{cc}
0 & \mathcal{S}-1 \\
1-\mathcal{S}^{-1} & 0
\end{array}\right), \quad f=-a \ln (1-u v)+b \ln \left(1+u v_{1}\right) .
$$

- Рекурсионный оператор:

$$
\begin{aligned}
\mathcal{R}= & \mathcal{H} \mathcal{J}= \\
= & \left(\begin{array}{cc}
-\frac{1}{\left(1-u_{1} v_{1}\right)^{2}} \mathcal{S}+\frac{1}{(1-u v)^{2}} & -\frac{u_{1}^{2}}{\left(1-u_{1} v_{1}\right)^{2}} \mathcal{S}+\frac{u^{2}}{(1-u v)^{2}}- \\
-\frac{2 u u_{1}}{\left(1-u_{1} v_{1}\right)(1-u v)} & -\frac{v_{-1}}{(1-u v)\left(1-u_{1} v_{1}\right)} \\
-\frac{v_{-1}^{2}}{\left(1-v_{-1}\right)^{2}} \mathcal{S}^{-1}-\frac{v^{2}}{(1-u v)^{2}} & -\frac{1}{\left(1-u_{-1} v_{-1}\right)^{2}} \mathcal{S}^{-1}+\frac{1-2 u v}{(1-u v)^{2}}
\end{array}\right)- \\
& -2 K_{1}(\mathcal{S}-1)^{-1}\left(\frac{v}{1-u v} \frac{u}{1-u v}\right)
\end{aligned}
$$

где симплектический оператор $\mathcal{J}$ задан выражением

$$
\mathcal{J}=\left(\begin{array}{cc}
0 & \frac{1}{1-u v} \\
-\frac{1}{1-u v} & 0
\end{array}\right)-\left(\begin{array}{c}
\frac{v}{1-u v} \\
\frac{u}{1-u v}
\end{array}\right)(\mathcal{S}+1)(\mathcal{S}-1)^{-1}\left(\begin{array}{cc}
\frac{v}{1-u v} & \left.\frac{u}{1-u v}\right)
\end{array}\right.
$$


- Нетривиальная симметрия:

$$
\mathcal{R}\left(K_{1}\right)=\left(\begin{array}{c}
\frac{1}{\left(1-u_{1} v_{1}\right)^{2}}\left(u_{1}-\frac{u_{2}}{1-u_{2} v_{2}}-\frac{u_{1}^{2} v}{1-u v}\right)- \\
-\frac{1}{(1-u v)^{2}}\left(u-\frac{u_{1}}{1-u_{1} v_{1}}-\frac{u^{2} v_{-1}}{1-u_{-1} v_{-1}}\right) \\
\frac{1}{(1-u v)^{2}}\left(v-\frac{u_{1} v^{2}}{1-u_{1} v_{1}}-\frac{v_{-1}}{1-u_{-1} v_{-1}}\right)- \\
-\frac{1}{\left(1-u_{-1} v_{-1}\right)^{2}}\left(v_{-1}-\frac{u v_{-1}^{2}}{1-u v}-\frac{v_{-2}}{1-u_{-2} v_{-2}}\right)
\end{array}\right) .
$$

- Представление Лакса [74]:

$$
\begin{aligned}
& M=\left(\begin{array}{cc}
\lambda+(1-\lambda) u v & u \\
(1-\lambda) v & 1
\end{array}\right) \\
& U=a\left(\begin{array}{cc}
\frac{\lambda-1}{2} & \frac{u}{1-u v} \\
\frac{(1-\lambda) v_{-1}}{1-u_{-1} v_{-1}} & -\frac{\lambda-1}{2}
\end{array}\right)+ \\
&+\frac{b}{2 \lambda\left(1+u_{-1} v\right)}\left(\begin{array}{cc}
(\lambda-1)\left(1-u_{-1} v\right) & 2 u_{-1} \\
2(1-\lambda) v & (1-\lambda)\left(1-u_{-1} v\right)
\end{array}\right) .
\end{aligned}
$$

Рекурсионный оператор $\mathcal{R}$ имеет затравку $\sigma=\left(\begin{array}{c}-u \\ v\end{array}\right)$, причем $\mathcal{R}(\sigma)=K_{1}$, если положить константу интегрирования равной нулю. Подобно цепочке Абловица-Ладека в п. 4.12 , коэффициенты перед $a$ и $b$, а именно $K_{1}$ и $K_{-1}$, являются коммутирующими симметриями уравнения. Более того, существует другой слабо нелокальный рекурсионный оператор

$$
\begin{aligned}
\mathcal{R}^{\prime}= & \mathcal{H} \mathcal{J}^{\prime}= \\
= & \left(\begin{array}{cc}
\frac{1}{\left(1+u_{-1} v\right)^{2}} \mathcal{S}^{-1}-\frac{1+2 u v_{1}}{\left(1+u v_{1}\right)^{2}} & -\frac{u^{2}}{\left(1+u v_{1}\right)^{2}} \mathcal{S}+\frac{u_{-1}^{2}}{\left(1+u u_{-1} v\right)^{2}}- \\
-\frac{v^{2}}{\left(1+u_{-1} v\right)^{2}} \mathcal{S}^{-1}-\frac{v_{1}^{2}}{\left(1+u v_{1}\right)^{2}} & \frac{1}{\left(1+u v_{1}\right)^{2}} \mathcal{S}-\frac{1}{\left(1+u u_{-1} v\right)^{2}}-\frac{2 u_{-1}}{\left(1+u v_{1}\right)} \\
-2 K_{-1}(\mathcal{S}-1)^{-1}\left(\begin{array}{cc}
\frac{v_{1}}{1+u v_{1}} & \frac{u_{-1}}{1+u_{-1} v}
\end{array}\right),
\end{array}\right.
\end{aligned}
$$


где симплектический оператор $\mathcal{J}^{\prime}$ имеет вид

$$
\begin{aligned}
& \mathcal{J}^{\prime}=\left(\begin{array}{cc}
0 & \frac{1}{1+u v_{1}}\left(\mathcal{S}-u_{-1} v_{1}\right) \frac{1}{1+u_{-1} v} \\
\frac{1}{1+u_{-1} v}\left(u_{-1} v_{1}-\mathcal{S}^{-1}\right) \frac{1}{1+u v_{1}} & 0
\end{array}\right)-
\end{aligned}
$$

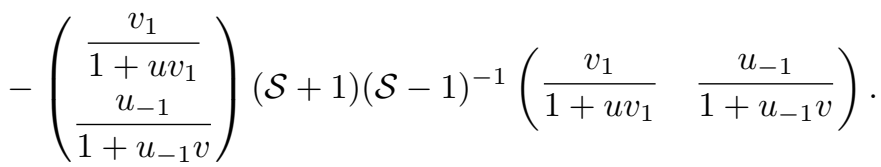

Снова $\sigma$ является затравкой для $\mathcal{R}^{\prime}$, причем $\mathcal{R}^{\prime}(\sigma)=K_{-1}$, с учетом соотношения

$$
(\mathcal{S}-1)^{-1}\left(-\frac{u v_{1}}{1+u v_{1}}+\frac{u_{-1} v}{1+u_{-1} v}\right)=-\frac{u_{-1} v}{1+u_{-1} v} .
$$

Проведя прямые вычисления, получим $\mathcal{J}^{\prime} \mathcal{R}=\mathcal{J R}^{\prime}=\mathcal{J}^{\prime}-\mathcal{J}$. Таким образом, эти два рекурсионных оператора удовлетворяют соотношению $\mathcal{R}^{\prime} \mathcal{R}=\mathcal{R} \mathcal{R}^{\prime}=\mathcal{R}^{\prime}-\mathcal{R}$, т. е. $\left(\mathcal{R}^{\prime}-\mathrm{id}\right)(\mathcal{R}-\mathrm{id})=(\mathcal{R}-\mathrm{id})\left(\mathcal{R}^{\prime}-\mathrm{id}\right)=\mathrm{id}$.

4.15. Цепочка Чена-Ли-Лю.

- Уравнение [74]:

$$
\left.\begin{array}{c}
u_{t}=a(1+u v)\left(u_{1}-u\right)+b\left(1+u_{-1} v\right)^{-1}\left(u-u_{-1}\right) \\
v_{t}=a(1+u v)\left(v-v_{-1}\right)+b\left(1+u v_{1}\right)^{-1}\left(v_{1}-v\right)
\end{array}\right\}:=a K_{1}+b K_{-1} .
$$

- Гамильтонова структура:

$$
\mathcal{H}=\left(\begin{array}{cc}
0 & 1+u v \\
-(1+u v) & 0
\end{array}\right), \quad f=a\left(u v_{-1}-u v\right)+b \ln \frac{1+u v}{1+u v_{1}} .
$$

- Рекурсионный оператор:

$$
\begin{aligned}
& \mathcal{R}=\mathcal{H}_{2} \mathcal{H}^{-1}=\left(\begin{array}{cc}
(1+u v) \mathcal{S}-2 u v+u_{1} v+u v_{-1} & u u_{1}-u^{2} \\
v^{2}-v v_{-1} & (1+u v) \mathcal{S}^{-1}
\end{array}\right)+ \\
& +K_{1}(\mathcal{S}-1)^{-1}\left(\begin{array}{cc}
\frac{v}{1+u v} & \frac{u}{1+u v}
\end{array}\right)-\left(\begin{array}{c}
u \\
-v
\end{array}\right)(\mathcal{S}-1)^{-1}\left(\begin{array}{ll}
v-v_{-1} & u-u_{1}
\end{array}\right),
\end{aligned}
$$

где гамильтонов оператор $\mathcal{H}_{2}$ задан выражением

$$
\begin{aligned}
& \mathcal{H}_{2}=\left(\begin{array}{cc}
0 & (1+u v)\left(\mathcal{S}(1+u v)-u v+u_{1} v\right) \\
\left(u v-u_{1} v-(1+u v) \mathcal{S}^{-1}\right)(1+u v) & 0
\end{array}\right)- \\
& -K_{1}(\mathcal{S}-1)^{-1}\left(\begin{array}{ll}
u & -v
\end{array}\right)-\left(\begin{array}{c}
u \\
-v
\end{array}\right) \mathcal{S}(\mathcal{S}-1)^{-1} K_{1}^{\mathrm{T}} .
\end{aligned}
$$

- Нетривиальная симметрия:

$$
\mathcal{R}\left(K_{1}\right)=\left(\begin{array}{c}
(1+u v)\left(u_{1} u_{2} v_{1}+u_{2}+u_{1}^{2} v+u u_{1} v_{-1}+\right. \\
\left.+u^{2} v-u_{1}-u^{2} v_{-1}-2 u u_{1} v-u_{1}^{2} v_{1}\right) \\
(1+u v)\left(u_{1} v^{2}+2 u v_{-1} v+u_{-1} v_{-1}^{2}+v_{-1}-v_{-2}-\right. \\
\left.-u v^{2}-u v_{-1}^{2}-u_{1} v_{-1} v-u_{-1} v_{-2} v_{-1}\right)
\end{array}\right) .
$$


- Представление Лакса [74]:

$$
\begin{aligned}
M & =\left(\begin{array}{cc}
\lambda+u v & u \\
(1-\lambda) v & 1
\end{array}\right), \\
U & =a\left(\begin{array}{cc}
\lambda-1+u v_{-1} & u \\
(1-\lambda) v_{-1} & 0
\end{array}\right)+\frac{b}{\lambda\left(1+u_{-1} v\right)}\left(\begin{array}{cc}
u_{-1} v & u_{-1} \\
(1-\lambda) v & 1-\lambda
\end{array}\right) .
\end{aligned}
$$

Коэффициенты перед $a$ и $b$, т. е. $K_{1}$ и $K_{-1}$, являются коммутирующими симметриями уравнения. Приведенный выше рекурсионный оператор $\mathcal{R}$ имеет затравку $\sigma=\left(\begin{array}{c}-u \\ v\end{array}\right)$, причем $\mathcal{R}(\sigma)=K_{1}$.

Существует обратный слабо нелокальный рекурсионный оператор

$$
\begin{aligned}
& \mathcal{R}^{\prime}=\mathcal{H}_{2}^{\prime} \mathcal{H}^{-1}=\left(\begin{array}{cc}
\frac{1+u v}{\left(1+u_{-1} v\right)^{2}} \mathcal{S}^{-1} & -\frac{u_{-1}\left(u-u_{-1}\right)}{\left(1+u_{-1} v\right)^{2}} \\
-\frac{v_{1}\left(v_{1}-v\right)}{\left(1+u v_{1}\right)^{2}} & \frac{1+u v}{\left(1+u v_{1}\right)^{2}} \mathcal{S}+\frac{v_{1} u-2 u_{-1} v_{1}+u_{-1} v}{\left(1+u_{-1} v\right)\left(1+u v_{1}\right)}
\end{array}\right)- \\
& -K_{-1}(\mathcal{S}-1)^{-1}\left(\frac{2 v_{1}}{1+u v_{1}}-\frac{v}{1+u v} \quad \frac{2 u_{-1}}{1+u_{-1} v}-\frac{u}{1+u v}\right)- \\
& -\left(\begin{array}{c}
-u \\
v
\end{array}\right)(\mathcal{S}-1)^{-1}\left(\frac{v_{1}}{1+u v_{1}}-\frac{v}{1+u v} \quad \frac{u_{-1}}{1+u_{-1} v}-\frac{u}{1+u v}\right),
\end{aligned}
$$

где гамильтонов оператор $\mathcal{H}_{2}^{\prime}$ имеет вид

$$
\begin{aligned}
& \mathcal{H}_{2}^{\prime}=\left(\begin{array}{cr}
\frac{1+u v}{1+u_{-1} v}\left(\mathcal{S}^{-1} \frac{1+u v}{1+u v_{1}}+\right. \\
0 & \left.+\frac{v_{1}\left(u-u_{-1}\right)}{1+u v_{1}}\right) \\
-\left(\frac{1+u v}{1+u v_{1}} \mathcal{S}+\right. & 0 \\
\left.+\frac{v_{1}\left(u-u_{-1}\right)}{1+u v_{1}}\right) \frac{1+u v}{1+u_{-1} v} &
\end{array}\right)- \\
& -K_{-1}(\mathcal{S}+1)(\mathcal{S}-1)^{-1} K_{-1}^{\mathrm{T}}- \\
& -K_{-1} \mathcal{S}(\mathcal{S}-1)^{-1}\left(\begin{array}{ll}
-u & v
\end{array}\right)-\left(\begin{array}{c}
-u \\
v
\end{array}\right)(\mathcal{S}-1)^{-1} K_{-1}^{\mathrm{T}} \text {. }
\end{aligned}
$$

Снова $\sigma$ является затравкой для $\mathcal{R}^{\prime}$.

4.16. Цепочка Абловица-Рамани-Сигура (Герджикова-Иванова).

- Уравнение [74]:

$$
\left.\begin{array}{c}
u_{t}=\left(a u_{1}-b u_{-1}\right)(1+u v)\left(1-u v_{1}\right) \\
v_{t}=\left(b v_{1}-a v_{-1}\right)(1+u v)\left(1-u_{-1} v\right)
\end{array}\right\}:=a K_{1}+b K_{-1} .
$$

- Симплектический оператор:

$$
\mathcal{J}=\left(\begin{array}{cc}
0 & \frac{1}{1-u v_{1}} \mathcal{S}-\frac{1}{1+u v} \\
\frac{1}{1+u v}-\mathcal{S}^{-1} \frac{1}{1-u v_{1}} & 0
\end{array}\right)
$$


причем

$$
\mathcal{J}\left(a K_{1}+b K_{-1}\right)=\delta_{(u, v)}\left(a\left(u v_{-1}-u v-u v u_{1} v_{1}\right)+b\left(u_{-1} v_{1}-u v_{1}+u_{-1} u v v_{1}\right)\right)
$$

- Гамильтонова структура:

$$
\begin{aligned}
\mathcal{H}=\left(\begin{array}{cc}
0 & (1+u v)\left(\mathcal{S}(1+u v)+u v_{-1}\right) \times \\
-\left(1-u_{-1} v\right)\left((1+u v) \mathcal{S}^{-1}+u v_{-1}\right) \times\left(1-u_{-1} v\right) \\
\times(1+u v)
\end{array}\right. \\
\quad-K_{1} \mathcal{S}(\mathcal{S}-1)^{-1}\left(\begin{array}{cc}
u & -v)-\left(\begin{array}{c}
u \\
-v
\end{array}\right)(\mathcal{S}-1)^{-1} K_{1}^{\mathrm{T}}
\end{array}\right)
\end{aligned}
$$

- Рекурсионный оператор:

$$
\begin{aligned}
& \mathcal{R}=\left(\begin{array}{cc}
(1+u v)\left(1-u v_{1}\right) \mathcal{S}+u_{1} v-u_{1} v_{1}+ & -u u_{1}(1+u v) \mathcal{S}-u^{2}\left(1+u_{-1} v_{-1}\right)+ \\
+u v_{-1}-u v\left(1+u_{-1} v_{-1}+2 u_{1} v_{1}\right) & +\frac{1-u v_{1}}{1-u_{-1} v} u_{1}\left(u-u_{-1}-2 u u_{-1} v\right) \\
-\left(1-u_{-1} v\right) v_{-1} v-(1+u v) v_{-1} v \mathcal{S}^{-1} & (1+u v)\left(1-u_{-1} v\right) \mathcal{S}^{-1}+u v u_{-1} v_{-1}
\end{array}\right)+ \\
& +\left(\begin{array}{c}
u_{1}(1+u v)\left(1-u v_{1}\right) \\
-v_{-1}(1+u v)\left(1-u_{-1} v\right)
\end{array}\right)(\mathcal{S}-1)^{-1} \times \\
& \times\left(\frac{v}{1+u v}-\frac{v_{1}}{1-u v_{1}} \frac{u}{1+u v}-\frac{u_{-1}}{1-u_{-1} v}\right)- \\
& -\left(\begin{array}{c}
u \\
-v
\end{array}\right)(\mathcal{S}-1)^{-1}\left(v-v_{-1}+u_{-1} v_{-1} v+u_{1} v v_{1} \quad u-u_{1}+u u_{-1} v_{-1}+u u_{1} v_{1}\right)= \\
& =\mathcal{H} \mathcal{J}+\left(\begin{array}{ll}
1 & 0 \\
0 & 1
\end{array}\right) \text {. }
\end{aligned}
$$

- Нетривиальная симметрия:

$$
\mathcal{R}\left(K_{1}\right)=\left(\begin{array}{c}
(1+u v)\left(1-u v_{1}\right)\left(\left(1+u_{1} v_{1}\right)\left(1-u_{1} v_{2}\right) u_{2}-u_{1}^{2} v_{1}(1+u v)+\right. \\
\left.+u u_{1} v_{-1}\left(1-u_{-1} v\right)-u_{1} v\left(u-u_{1}\right)\right) \\
(1+u v)\left(u_{-1} v-1\right)\left(\left(1+u_{-1} v_{-1}\right)\left(1-u_{-2} v_{-1}\right) v_{-2}-u_{-1} v_{-1}^{2}(1+u v)+\right. \\
\left.+u_{1} v_{-1} v\left(1-u v_{1}\right)+u v_{-1}\left(v_{-1}-v\right)\right)
\end{array}\right) .
$$

- Представление Лакса [74]:

$$
\begin{aligned}
M= & \left(\begin{array}{cc}
\lambda+u v & u \\
(1-\lambda)\left(1-u v_{1}\right) v & 1-u v_{1}
\end{array}\right), \\
U= & a\left(\begin{array}{cc}
\lambda+u v_{-1}\left(1-u_{-1} v\right) & u \\
(1-\lambda)\left(1-u_{-1} v\right) v_{-1} & -u v
\end{array}\right)+ \\
& \quad+\frac{b}{\lambda}\left(\begin{array}{cc}
u_{-1} v-\lambda \\
(1-\lambda)\left(1-u_{-1} v\right) v & (1-\lambda)\left(1-u_{-1} v\right)-\lambda u_{-1} v_{1}(1+u v)
\end{array}\right) .
\end{aligned}
$$


Полученное в работе [74] уравнение имеет вид

$$
\left(\begin{array}{l}
u_{t} \\
v_{t}
\end{array}\right)=(a-b)\left(\begin{array}{c}
-u \\
v
\end{array}\right)+a K_{1}+b K_{-1} .
$$

Поскольку вектор $\sigma=\left(\begin{array}{c}-u \\ v\end{array}\right)$ коммутирует как с $K_{1}$, так и с $K_{-1}$, мы удалим эти члены из наших вычислений. Существует другой слабо нелокальный рекурсионный оператор

$$
\begin{aligned}
& \mathcal{R}^{\prime}=\left(\begin{array}{cc}
(1+u v)\left(1-u v_{1}\right) \mathcal{S}^{-1}+u v u_{-1} v_{1} & -1-u v_{1} \\
& -\frac{1-u v}{1-u_{-1} v} u_{-1}\left(u-u u_{-2}\left(1+u_{-1}-2 u u_{-1} v\right)\right. \\
\left(1-u_{-1} v\right) v_{1} v+(1+u v) v_{1} v \mathcal{S}^{-1} & (1+u v)\left(1-u_{-1} v\right) \mathcal{S}+u v_{1}-2 u_{-1} u v v_{1}- \\
& -u_{-1} v_{1}+u_{-1} v-u_{-2} v\left(1+u_{-1} v_{-1}\right)
\end{array}\right)+ \\
& +\left(\begin{array}{c}
-u_{-1}(1+u v)\left(1-u v_{1}\right) \\
v_{1}(1+u v)\left(1-u_{-1} v\right)
\end{array}\right)(\mathcal{S}-1)^{-1} \times \\
& \times\left(\frac{v}{1+u v}-\frac{v_{1}}{1-u v_{1}} \quad \frac{u}{1+u v}-\frac{u_{-1}}{1-u_{-1} v}\right)+\left(\begin{array}{c}
u \\
-v
\end{array}\right)(\mathcal{S}-1)^{-1} \times \\
& \times\left(v_{2}\left(1+u_{1} v_{1}\right)+v_{1}\left(u_{-1} v-1\right) \quad u_{-2}\left(1+u_{-1} v_{-1}\right)+u_{-1}\left(u v_{1}-1\right)\right)= \\
& =\mathcal{H}^{\prime} \mathcal{J}+\left(\begin{array}{ll}
1 & 0 \\
0 & 1
\end{array}\right)
\end{aligned}
$$

где гамильтонов оператор $\mathcal{H}^{\prime}$ имеет вид

$$
\begin{aligned}
& \mathcal{H}^{\prime}=\left(\begin{array}{cc}
0 & -(1+u v)\left(1-u_{-1} v\right) \\
(1+u v)\left(1-u_{-1} v\right) & 0
\end{array}\right)- \\
& -K_{-1} \mathcal{S}(\mathcal{S}-1)^{-1}\left(\begin{array}{ll}
u & -v
\end{array}\right)-\left(\begin{array}{c}
u \\
-v
\end{array}\right)(\mathcal{S}-1)^{-1} K_{-1}^{\mathrm{T}} .
\end{aligned}
$$

Оператор $\mathcal{R}^{\prime}$ является обратным к $\mathcal{R}$. Вектор $\sigma$ является затравкой для каждого из них, при этом $\mathcal{R}^{\prime}\left(K_{-1}\right)$ имеет вид

$$
\mathcal{R}^{\prime}\left(K_{-1}\right)=\left(\begin{array}{c}
\left(1-u v_{1}\right)(1+u v)\left(\left(1+u_{-1} v_{-1}\right)\left(1-u_{-1} v\right) u_{-2}-u u_{-1} v_{2}\left(1+u_{1} v_{1}\right)-\right. \\
\left.-u_{-1}^{2} v_{1}(1+u v)+u_{-1}\left(u_{-1} v+u v_{1}\right)\right) \\
\left(1-u_{-1} v\right)(1+u v)\left(-\left(1+u_{1} v_{1}\right)\left(1-u v_{1}\right) v_{2}+u_{-2} v v_{1}\left(1+u_{-1} v_{-1}\right)+\right. \\
\left.+u_{-1} v_{1}\left(v_{1} v u+v_{1}-v\right)-u v_{1}^{2}\right)
\end{array}\right) .
$$

\subsection{7. Ферромагнитная цепочка Гейзенберга.}

- Уравнение [14]:

$$
\begin{aligned}
& u_{t}=(u-v)\left(u-u_{1}\right)\left(u_{1}-v\right)^{-1} \\
& v_{t}=(u-v)\left(v_{-1}-v\right)\left(u-v_{-1}\right)^{-1}
\end{aligned}
$$

- Гамильтонова структура:

$$
\mathcal{H}=\left(\begin{array}{cc}
0 & (u-v)^{2} \\
-(u-v)^{2} & 0
\end{array}\right), \quad f=\ln (u-v)-\ln \left(u-v_{-1}\right) .
$$


- Симплектический оператор:

$$
\begin{aligned}
& \mathcal{J}=\left(\begin{array}{c}
0 \\
\left(u_{1}-v\right)^{-2} \mathcal{S}- \\
-\frac{\left(u-u_{1}\right)\left(v-v_{-1}\right)}{(u-v)^{2}\left(u-v_{-1}\right)\left(u_{1}-v\right)}
\end{array}\right. \\
& -\left(u-v_{-1}\right)^{-2} \mathcal{S}^{-1}+ \\
& +\frac{\left(u-u_{1}\right)\left(v-v_{-1}\right)}{(u-v)^{2}\left(u-v_{-1}\right)\left(u_{1}-v\right)} \\
& -\left(\frac{v-v_{-1}}{\frac{(u-v)\left(u-v_{-1}\right)}{(u-v)\left(u_{1}-v\right)}}\right)(\mathcal{S}+1)(\mathcal{S}-1)^{-1}\left(\frac{v-v_{-1}}{(u-v)\left(u-v_{-1}\right)} \quad \frac{u-u_{1}}{(u-v)\left(u_{1}-v\right)}\right) .
\end{aligned}
$$

- Рекурсионный оператор:

$$
\begin{aligned}
& \mathcal{R}=\mathcal{H} \mathcal{J}=\left(\begin{array}{cc}
\frac{(u-v)^{2}}{\left(u_{1}-v\right)^{2}} \mathcal{S}-\frac{2\left(u-u_{1}\right)\left(v-v_{-1}\right)}{\left(u-v_{-1}\right)\left(u_{1}-v\right)} & -\frac{\left(u-u_{1}\right)^{2}}{\left(u_{1}-v\right)^{2}} \\
\frac{\left(v-v_{-1}\right)^{2}}{\left(u-v_{-1}\right)^{2}} & \frac{(u-v)^{2}}{\left(u-v_{-1}\right)^{2}} \mathcal{S}^{-1}
\end{array}\right)- \\
& -2\left(\begin{array}{l}
u_{t} \\
v_{t}
\end{array}\right)(\mathcal{S}-1)^{-1}\left(\frac{v-v_{-1}}{(u-v)\left(u-v_{-1}\right)} \quad \frac{u-u_{1}}{(u-v)\left(u_{1}-v\right)}\right) \text {. }
\end{aligned}
$$

- Нетривиальная симметрия:

$$
\begin{aligned}
\mathcal{R}\left(\begin{array}{l}
u_{t} \\
v_{t}
\end{array}\right) & = \\
= & \left(\begin{array}{c}
\frac{u-v}{\left(u_{1}-v\right)^{2}}\left(\frac{(u-v)\left(u_{1}-v_{1}\right)\left(u_{1}-u_{2}\right)}{u_{2}-v_{1}}+\frac{\left(u-u_{1}\right)^{2}\left(v_{-1}-v\right)}{u-v_{-1}}\right) \\
\frac{u-v}{\left(u-v_{-1}\right)^{2}}\left(\frac{(u-v)\left(u_{-1}-v_{-1}\right)\left(v_{-2}-v_{-1}\right)}{u_{-1}-v_{-2}}+\frac{\left(v-v_{-1}\right)^{2}\left(u-u_{1}\right)}{u_{1}-v}\right)
\end{array}\right) .
\end{aligned}
$$

- Представление Лакса:

$$
\begin{aligned}
M & =\left(\begin{array}{cc}
\lambda-2 u(u-v)^{-1} & -2(u-v)^{-1} \\
2 u v(u-v)^{-1} & \lambda+2 v(u-v)^{-1}
\end{array}\right), \\
U & =\lambda^{-1}\left(u-v_{-1}\right)^{-1}\left(\begin{array}{cc}
u+v_{-1} & 2 \\
-2 u v_{-1} & -\left(u+v_{-1}\right)
\end{array}\right) .
\end{aligned}
$$

Рекурсионный оператор $\mathcal{R}$ имеет слабо нелокальный обратный оператор

$$
\begin{aligned}
& \mathcal{R}^{-1}=\mathcal{H} \mathcal{J}^{\prime}=\left(\begin{array}{cc}
\frac{(u-v)^{2}}{(u-v)^{2}} \mathcal{S}^{-1} & \frac{\left(u-u_{-1}\right)^{2}}{\left(u_{-1}-v\right)^{2}} \\
-\frac{\left(v-v_{1}\right)^{2}}{\left(u-v_{1}\right)^{2}} & \frac{(u-v)^{2}}{\left(u-v_{1}\right)^{2}} \mathcal{S}-\frac{2\left(u-u_{-1}\right)\left(v-v_{1}\right)}{\left(u-v_{1}\right)\left(u_{-1}-v\right)}
\end{array}\right)-
\end{aligned}
$$

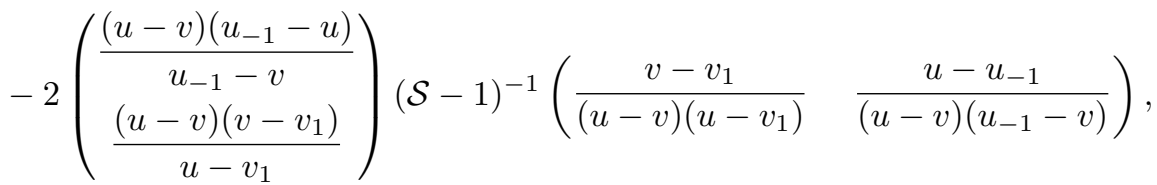


где симплектический оператор $\mathcal{J}^{\prime}$ задан выражением

$$
\begin{aligned}
& \mathcal{J}^{\prime}=\left(\begin{array}{cc}
0 & -\left(u-v_{1}\right)^{-2} \mathcal{S}+ \\
\left(u_{-1}-v\right)^{-2} \mathcal{S}^{-1}- & +\frac{\left(u-u_{-1}\right)\left(v-v_{1}\right)}{(u-v)^{2}\left(u-v_{1}\right)(u-1-v)} \\
-\frac{\left(u-u_{-1}\right)\left(v-v_{1}\right)}{(u-v)^{2}\left(u-v_{1}\right)\left(u_{-1}-v\right)} & 0
\end{array}\right)+ \\
& +\left(\begin{array}{c}
\frac{v-v_{1}}{(u-v)\left(u-v_{1}\right)} \\
\frac{u-u_{-1}}{(u-v)\left(u_{-1}-v\right)}
\end{array}\right)(\mathcal{S}+1)(\mathcal{S}-1)^{-1}\left(\frac{v-v_{1}}{(u-v)\left(u-v_{1}\right)} \quad \frac{u-u_{-1}}{(u-v)\left(u_{-1}-v\right)}\right) .
\end{aligned}
$$

Ферромагнитная цепочка Гейзенберга является частным случаем (если положить $a=1, b=0$ и $\left.h(u, v)=(u-v)^{2} / 2\right)$ цепочки Ландау-Лифшица (Склянина) [14], [75], [76]:

$$
\begin{aligned}
& u_{t}=a\left(\frac{2 h}{u_{1}-v}+h_{v}\right)+b\left(\frac{2 h}{u_{-1}-v}+h_{v}\right), \\
& v_{t}=a\left(\frac{2 h}{u-v_{-1}}-h_{u}\right)+b\left(\frac{2 h}{u-v_{1}}-h_{u}\right),
\end{aligned}
$$

где

$$
h(u, v)=\frac{i}{4}\left(K_{1}(1-u v)^{2}-K_{2}(1+u v)^{2}+K_{3}(u+v)^{2}\right), \quad K_{1} K_{2} K_{3} \neq 0, \quad K_{n} \in \mathbb{R} .
$$

Отметим, что коэффициенты при $a$ и $b$ в цепочке Ландау-Лифшица (Склянина) (87) коммутируют.

Представление Лакса для уравнения (87) получено в работе [75] в виде

$$
\begin{aligned}
& U=i \sum_{k=1}^{3} a N_{k}^{+}(\lambda) S_{k}\left(u, v_{-1}\right) \sigma_{k}+b N_{k}^{-}(\lambda) S_{k}\left(v, u_{-1}\right) \sigma_{k}, \\
& M=\frac{1}{\sqrt{\langle\mathbf{S K S}\rangle}}\left(I-\sum_{k=1}^{3} M_{k}(\lambda) S_{k}(u, v) \sigma_{k}\right) .
\end{aligned}
$$

Здесь $\sigma_{k}-$ матрицы Паули:

$$
\sigma_{1}=\left(\begin{array}{cc}
0 & 1 \\
1 & 0
\end{array}\right), \quad \sigma_{2}=\left(\begin{array}{cc}
0 & -i \\
i & 0
\end{array}\right), \quad \sigma_{3}=\left(\begin{array}{cc}
1 & 0 \\
0 & -1
\end{array}\right)
$$


вектор-функция $S(p, q)$ задана следующим образом:

$$
\left(S_{1}(p, q), S_{2}(p, q), S_{3}(p, q)\right)=\left(\frac{1-p q}{p-q}, \frac{i+i p q}{p-q}, \frac{p+q}{p-q}\right),
$$

причем она удовлетворяет условию $S_{1}^{2}+S_{2}^{2}+S_{3}^{2}=1$,

$$
\langle\mathbf{S K S}\rangle=\sum_{l=1}^{3} K_{l} S_{l}^{2}(u, v)
$$

a $M_{l}(\lambda), N_{l}^{ \pm}(\lambda)$ можно выразить через эллиптические функции Якоби спектрального параметра $\lambda$ :

$$
\begin{array}{ll}
M_{1}(\lambda)=\sqrt{1-K_{1} K_{2}^{-1}} \operatorname{sn}(\lambda, \kappa), & N_{1}^{ \pm}(\lambda)=\frac{\sqrt{K_{2} K_{3}}}{2} M_{1}(\lambda \pm \mu), \\
M_{2}(\lambda)=\sqrt{1-K_{2} K_{1}^{-1}} \operatorname{cn}(\lambda, \kappa), & N_{2}^{ \pm}(\lambda)=\frac{\sqrt{K_{1} K_{3}}}{2 \kappa} M_{2}(\lambda \pm \mu), \\
M_{3}(\lambda)=\sqrt{1-K_{3} K_{1}^{-1}} \operatorname{dn}(\lambda, \kappa), & N_{3}^{ \pm}(\lambda)=\frac{\sqrt{K_{1} K_{2}}}{2} M_{3}(\lambda \pm \mu),
\end{array}
$$

где $\kappa$ и $\mu$ заданы уравнениями

$$
\kappa=\sqrt{\frac{K_{3}\left(K_{1}-K_{2}\right)}{K_{2}\left(K_{1}-K_{3}\right)}}, \quad \operatorname{cn}(\mu, \kappa)=\frac{K_{1}}{K_{3}} .
$$

Вместо явной униформизации эллиптической кривой можно воспользоваться тождествами

$$
\begin{gathered}
\frac{M_{l}^{2}-1}{K_{l}}=\frac{M_{j}^{2}-1}{K_{j}}, \quad N_{l}^{ \pm}=\frac{K_{1}}{2\left(M_{1}^{2}-1\right)}\left(M_{l} \pm M_{j} M_{k}\right), \\
l, j, k \in\{1,2,3\}, \quad l \neq j \neq k \neq l .
\end{gathered}
$$

Один локальный гамильтонов оператор уравнения (87) имеет вид [75]

$$
\mathcal{H}=h(u, v)\left(\begin{array}{cc}
0 & 1 \\
-1 & 0
\end{array}\right), \quad f=a \ln \frac{h(u, v)}{\left(u_{1}-v\right)^{2}}+b \ln \frac{h(u, v)}{\left(u_{-1}-v\right)^{2}}
$$

Зная представление Лакса, можно, в принципе, посчитать его рекурсионный оператор (как сделано в п. 3.2) с множителями

$$
\mu_{ \pm}(\lambda)=\left(N_{1}^{ \pm}(\lambda)\right)^{2}, \quad \nu_{ \pm}(\lambda)=N_{1}^{ \pm}(\lambda) N_{2}^{ \pm}(\lambda) N_{3}^{ \pm}(\lambda) .
$$

Однако соответствующие вычисления довольно громоздки и мы не получили компактные представления для этих операторов. 


\subsection{8. Цепочка Белова-Чалтикяна.}

- Уравнение [77]:

$$
\begin{aligned}
& u_{t}=u\left(v_{2}-v_{-1}\right), \\
& v_{t}=u_{-1}-u+v\left(v_{1}-v_{-1}\right) .
\end{aligned}
$$

- Гамильтонова структура [77]:

$$
\begin{array}{rl}
\mathcal{H}_{1}= & \left(\begin{array}{cc}
u\left(\mathcal{S}-\mathcal{S}^{-1}\right)\left(\mathcal{S}+1+\mathcal{S}^{-1}\right) u & u(\mathcal{S}-1)\left(\mathcal{S}+1+\mathcal{S}^{-1}\right) v \\
v\left(1-\mathcal{S}^{-1}\right)\left(\mathcal{S}+1+\mathcal{S}^{-1}\right) u & v\left(\mathcal{S}-\mathcal{S}^{-1}\right) v+\mathcal{S}^{-1} u-u \mathcal{S}
\end{array}\right), \quad f_{1}=v, \\
0 & u\left(1+\mathcal{S}+\mathcal{S}^{2}\right)\left(u \mathcal{S}-\mathcal{S}^{-2} u\right) \\
\mathcal{H}_{2}= & \left.\begin{array}{cc}
\left(u \mathcal{S}^{2}-\mathcal{S}^{-1} u\right)\left(1+\mathcal{S}^{-1}+\mathcal{S}^{-2}\right) u & v(1+\mathcal{S})\left(u \mathcal{S}-\mathcal{S}^{-2} u\right)+ \\
+\left(u \mathcal{S}^{2}-\mathcal{S}^{-1} u\right)\left(1+\mathcal{S}^{-1}\right) v
\end{array}\right)+ \\
& +\left(\begin{array}{cc}
u\left(1+\mathcal{S}+\mathcal{S}^{2}\right)\left(\mathcal{S}^{-1} v-v \mathcal{S}\right) \times & u\left(1+\mathcal{S}+\mathcal{S}^{2}\right)\left(\mathcal{S}^{-1} v-v \mathcal{S}\right)\left(1+\mathcal{S}^{-1}\right) v \\
\times\left(1+\mathcal{S}^{-1}+\mathcal{S}^{-2}\right) u & \\
v(1+\mathcal{S})\left(\mathcal{S}^{-1} v-v \mathcal{S}\right) \times & v(1+\mathcal{S})\left(\mathcal{S}^{-1} v-v \mathcal{S}\right)\left(1+\mathcal{S}^{-1}\right) v \\
\times\left(1+\mathcal{S}^{-1}+\mathcal{S}^{-2}\right) u &
\end{array}\right),
\end{array}
$$

$f_{2}=-\frac{1}{3} \ln u$.

- Нетривиальная симметрия:

$$
\mathcal{H}_{2} \delta f_{1}=\left(\begin{array}{c}
u v_{-1}\left(v+v_{-1}+v_{-2}\right)-u v_{2}\left(v_{1}+v_{2}+v_{3}\right)+u\left(u_{1}+u_{2}-u_{-1}-u_{-2}\right) \\
\left(u-v v_{1}\right)\left(v+v_{1}+v_{2}\right)+\left(v v_{-1}-u_{-1}\right)\left(v+v_{-1}+v_{-2}\right)-v\left(u_{-2}-u_{1}\right)
\end{array}\right) .
$$

- Мастер-симметрия [78]:

$$
\left(\begin{array}{c}
n u_{t}+u v_{1}+4 u v_{-1}+u v \\
n v_{t}+u-v v_{1}-4 u_{-1}+4 v v_{-1}+v^{2}
\end{array}\right) .
$$

- Представление Лакса [79]:

$$
M=\left(\begin{array}{ccc}
\lambda & \lambda v & \lambda u_{-1} \\
1 & 0 & 0 \\
0 & 1 & 0
\end{array}\right), \quad U=\left(\begin{array}{ccc}
v-\lambda & -\lambda v & -\lambda u_{-1} \\
-1 & v_{-1} & 0 \\
0 & -1 & v_{-2}
\end{array}\right) .
$$

Цепочка Белова-Чалтикяна - это цепочка Буссинеска, связанная с дискретной алгеброй $W_{3}[79]$.

В недавней работе [80] авторы выписали цепочку Буссинеска, связанную с дискретной алгеброй $W_{m}$, для зависимых переменных $u^{1}, u^{2}, \ldots, u^{m}$ и независимых переменных $n$ и $t$ в следующем виде:

$$
\begin{aligned}
u_{t}^{1} & =-u^{1}\left(u_{m}^{2}-u_{-1}^{2}\right), \\
u_{t}^{i} & =u^{i+1}-u_{-1}^{i+1}-u^{i}\left(u_{i-1}^{2}-u_{-1}^{2}\right), \quad i=2,3, \ldots, m-1, \\
u_{t}^{m} & =u^{1}-u_{-1}^{1}-u^{m}\left(u_{m-1}^{2}-u_{-1}^{2}\right) .
\end{aligned}
$$


Вектор $\boldsymbol{\tau}=\left(\tau^{1}, \ldots, \tau^{n}\right)^{\mathrm{T}}$, заданный как

$$
\begin{aligned}
\tau^{1} & =n u_{t}^{1}-u^{1}\left((m+1) u_{m}^{2}+\sum_{l=0}^{m} u_{l}^{2}\right), \\
\tau^{i} & =n u_{t}^{i}-u^{i}\left(i u_{i-1}^{2}+\sum_{l=0}^{i-1} u_{l}^{2}\right)+(i+1) u^{i+1}, \quad i=2,3, \ldots, m-1, \\
\tau^{m} & =n u_{t}^{n}-u^{m}\left(m u_{m-1}^{2}+\sum_{l=0}^{m-1} u_{l}^{2}\right)+(m+1) u^{1},
\end{aligned}
$$

является мастер-симметрией. Его гамильтоновы структуры изучены также в работе [80].

\subsection{9. Цепочка Блажака-Марсиньяка.}

- Уравнение [81]:

$$
\begin{aligned}
u_{t} & =w_{1}-w_{-1}, \\
v_{t} & =u_{-1} w_{-1}-u w, \\
w_{t} & =w\left(v-v_{1}\right) .
\end{aligned}
$$

- Гамильтонова структура [81]:

$$
\begin{aligned}
& \mathcal{H}_{1}=\left(\begin{array}{ccc}
\mathcal{S}-\mathcal{S}^{-1} & 0 & 0 \\
0 & 0 & \left(\mathcal{S}^{-1}-1\right) w \\
0 & -w(\mathcal{S}-1) & 0
\end{array}\right), \quad f_{1}=u w+\frac{1}{2} v^{2}, \\
& \mathcal{H}_{2}=\left(\begin{array}{ccc}
\mathcal{S} v-v \mathcal{S}^{-1}- & \mathcal{S} w \mathcal{S}-\mathcal{S}^{-1} w & u(\mathcal{S}+1)^{-1}(1-\mathcal{S}) w \\
-u(\mathcal{S}+1)^{-1}(1-\mathcal{S}) u & & \\
w \mathcal{S}-\mathcal{S}^{-1} w \mathcal{S}^{-1} & \mathcal{S}^{-1} u w-u w \mathcal{S} & v\left(\mathcal{S}^{-1}-1\right) w \\
w(\mathcal{S}+1)^{-1}(1-\mathcal{S}) u & -w(\mathcal{S}-1) v & w\left(\mathcal{S}^{-1}-\mathcal{S}\right) w- \\
& & -w(\mathcal{S}+1)^{-1}(1-\mathcal{S}) w
\end{array}\right) \\
& f_{2}=v \text {. }
\end{aligned}
$$

- Рекурсионный оператор:

$$
\mathcal{R}=\mathcal{H}_{2} \mathcal{H}_{1}^{-1}
$$

где

$$
\mathcal{H}_{1}^{-1}=\left(\begin{array}{ccc}
\frac{1}{2}(\mathcal{S}-1)^{-1}+\frac{1}{2}(\mathcal{S}+1)^{-1} & 0 & 0 \\
0 & 0 & -(\mathcal{S}-1)^{-1} \frac{1}{w} \\
0 & -\frac{1}{w} \mathcal{S}(\mathcal{S}-1)^{-1} & 0
\end{array}\right) .
$$

- Нетривиальная симметрия:

$$
\mathcal{H}_{2} \delta f_{1}=\mathcal{H}_{2}\left(\begin{array}{c}
w \\
v \\
u
\end{array}\right)=\left(\begin{array}{c}
w_{1}\left(v_{1}+v_{2}\right)-w_{-1}\left(v+v_{-1}\right) \\
u_{-1} w_{-1}\left(v+v_{-1}\right)-u w\left(v+v_{1}\right)-w_{-1} w_{-2}+w w_{1} \\
w\left(v^{2}-v_{1}^{2}\right)+w\left(w_{-1} u_{-1}-w_{1} u_{1}\right)
\end{array}\right)
$$


- Мастер-симметрия:

$$
\mathcal{R}\left(\begin{array}{c}
u / 2 \\
v \\
3 w / 2
\end{array}\right)
$$

- Представление Лакса [81]:

$$
L=\mathcal{S}^{2}+u_{1} \mathcal{S}-v_{1}+w \mathcal{S}^{-1}, \quad A=\mathcal{S}^{2}+u_{1} \mathcal{S}-v_{1} .
$$

Мы не выписываем явно рекурсионный оператор для этой цепочки, который уже не является слабо нелокальным, хотя оба оператора $\mathcal{H}_{2}$ и $\mathcal{H}_{1}^{-1}$ слабо нелокальные. Утверждение, что такой рекурсионный оператор генерирует локальные симметрии, доказывается так же, как было сделано в работе [31] для слабо нелокальных дифференциальных рекурсионных операторов. Можно вычислить следующий гамильтониан, равный $u v w+u v_{1} w+v^{3} / 3-w w_{1}$. Мастер-симметрия этой цепочки сильно нелокальна [78].

Еще одна трехкомпонентная цепочка представлена в работе [29] в виде

$$
\begin{aligned}
p_{t} & =q_{1}-q, \\
q_{t} & =q\left(p_{-1}-p\right)+r-r_{-1}, \\
r_{t} & =r\left(p_{-1}-p_{1}\right) .
\end{aligned}
$$

У нее есть представление Лакса

$$
M=\left(\begin{array}{ccc}
0 & 1 & 0 \\
q & u+\lambda & 1 \\
r & 0 & 0
\end{array}\right), \quad U=\left(\begin{array}{ccc}
-p_{-1} & 1 & 0 \\
q & \lambda & 1 \\
r_{-1} & 0 & -p
\end{array}\right)
$$

Эта цепочка связана с цепочкой Блажака-Марсиньяка преобразованием Миуры

$$
p=v_{1}, \quad q=-u w, \quad r=-w w_{1} .
$$

\section{5. ЗАКЛЮЧЕНИЕ}

В настоящей работе представлен обзор двух близких концепций, непосредственно связанных с представлением Лакса для интегрируемых систем: преобразований Дарбу и рекурсионных операторов. В качестве двух типичных примеров выбраны хорошо известное нелинейное уравнение Шредингера, представление Лакса которого полиномиально по спектральному параметру, и деформация нелинейного уравнения Шредингера с производной, представление Лакса которого инвариантно относительно диэдральной группы редукций $\mathbb{D}_{2}$. В работе нами предложен список интегрируемых дифференциально-разностных уравнений, включающий сами уравнения, их гамильтоновы структуры, рекурсионные операторы, нетривиальные обобщенные симметрии и представления Лакса. Для большинства уравнений сделаны также замечания относительно их связей с другими известными уравнениями и о слабо нелокальном обратном рекурсионном операторе, если такой существует.

Теория интегрируемых разностных (или дискретных) уравнений представляет собой сравнительно новую, но очень активную область исследований. Благодаря работам Адлера, Бобенко и Суриса появилась классификация интегрируемых 
аффинно-линейных четырехточечных уравнений [82], [83], основанная на свойстве 3D-совместности. Затем Леви и Ямилов предложили использовать существование обобщенной симметрии в качестве критерия интегрируемости [84] для классификации интегрируемых разностных уравнений. Они расширили список уравнений Адлера-Бобенко-Суриса. Симметрийные потоки всех интегрируемых разностных уравнений можно считать интегрируемыми дифференциально-разностными уравнениями. Понятие рекурсионного оператора распространено на разностные уравнения и показано, что он генерирует бесконечную последовательность симметрий и канонических законов сохранения для разностных уравнений [35], [11], [53]. Можно доказать, что разностное уравнение и его симметрийные потоки имеют общий рекурсионный оператор. Таким образом, изучение симметрийной структуры интегрируемых разностных уравнений по сути то же самое, что и изучение интегрируемых дифференциально-разностных уравнений. Это одна из мотиваций создания предложенного в этой работе списка. Кроме того, этот список может служить эталонным тестом для развития компьютерного программного обеспечения для символьных расчетов рекурсионных операторов, представлений Лакса, симметрий и законов сохранения для нелинейных дифференциально-разностных уравнений [72].

Мы не предоставили здесь строгого доказательства того, что построенные нами с помощью преобразований Дарбу рекурсионные операторы являются операторами Нийенхейса для уравнений, полученных из соответствующих представлений Лакса. Для полиномиальных по спектральному параметру представлений Лакса, при соблюдении некоторых формальных технических условий, набросок доказательства дан в работе [29]. Мы уверены, что основное утверждение работы [29] можно существенно обобщить и упростить, а также существует четкое строгое алгебраическое доказательство того, что операторы, полученные из преобразований Дарбу, инвариантные относительно групп редукций, являются в действительности рекурсионными операторами Нийенхейса.

Благодарности. А. В. Михайлов выражает признательность за частичную финанасовую поддержку EPSRC (грант EP/I038675/1), а также ФЦП "Научные и педагогические кадры инновационной России на 2009-2013 гг.” (соглашение 8499), Дж. П. Ванг благодарна за финансовую поддержку EPSRC (грант EP/I038659/1).

\section{Список литературы}

[1] H. D. Wahlquist, F. B. Estabrook, Phys. Rev. Lett., 31:23 (1973), 1386-1390.

[2] S. Lombardo, A. V. Mikhailov, J. Phys. A, 37:31 (2004), 7727-7742, arXiv: nlin/0404013.

[3] S. Lombardo, A.V. Mikhailov, Commun. Math. Phys., 258:1 (2005), 179-202, arXiv: math-ph/0407048.

[4] S. Lombardo, Reductions of integrable equations and automorphic Lie algebra, PhD Thesis, University of Leeds, Leeds, 2004.

[5] R. T. Bury, Automorphic Lie algebras, corresponding integrable systems and their soliton solutions, PhD Thesis, University of Leeds, Leeds, 2010.

[6] А. В. Михайлов, Писъма в ЖКЭТФ, 30:7 (1979), 443-448.

[7] А. В. Михайлов, Писъма в ЖЭТФ, 32:2 (1980), 187-192.

[8] A. V. Mikhailov, Phys. D, 3:1-2 (1981), 73-117. 
[9] C. Rogers, W. K. Schief, Bäcklund and Darboux Transformations. Geometry and Modern Applications in Soliton Theory, Cambridge Texts in Applied Mathematics, 30, Cambridge Univ. Press, Cambridge, 2002.

[10] V. Volterra, Leçons sur la théorie mathématique de la lutte pour la vie, Gauthier-Villars, Paris, 1931.

[11] A. V. Mikhailov, J.P. Wang, P. Xenitidis, Nonlinearity, 24:7 (2011), 2079-2097; arXiv: 1009.2403.

[12] A. Ya. Maltsev, S. P. Novikov, Phys. D, 156:1-2 (2001), 53-80.

[13] J. P. Wang, Stud. Appl. Math., 129:3 (2012), 309-327.

[14] Е. К. Склянин, Функи. анализ и его прил., 16:4 (1982), 27-34.

[15] H. Zhang, G.-Z. Tu, W. Oevel, B. Fuchssteiner, J. Math. Phys., $32: 7$ (1991), 1908-1918.

[16] J.-P. Wang, J. Nonlinear Math. Phys., 9, suppl. 1 (2002), 213-233.

[17] В. Е. Захаров, С. В. Манаков, С.П. Новиков, Л.П. Питаевский, Теория солитонов: метод обратной задачи, Наука, М., 1980.

[18] М. Абловиц, Х. Сигур, Солитоны и метод обратной задачи, Мир, М., 1987.

[19] M. J. Ablowitz, P. A. Clarkson, Solitons, Nonlinear Evolution Equations and Inverse Scattering, London Mathematical Society Lecture Note Series, 149, Cambridge Univ. Press, Cambridge, 1991.

[20] A. V. Mikhailov, A. B. Shabat, V. V. Sokolov, "The symmetry approach to classification of integrable equations", What is integrability?, ed. V.E. Zakharov, Springer, Berlin, 1991, $115-184$.

[21] A. V. Mikhailov, A. B. Shabat, R. I. Yamilov, Commun. Math. Phys., 115:1 (1988), 1-19.

[22] В. Э. Адлер, А. Б. Шабат, Р. И. Ямилов, ТМФ, 125:3 (2000), 355-424.

[23] J. A. Sanders, J. P. Wang, J. Diff. Eq., 147:2 (1998), 410-434.

[24] В. Е. Захаров, А. Б. Шабат, ЖКЭТФ, 61:1 (1971), 118-134.

[25] M. Gürses, A. Karasu, V. V. Sokolov from Lax representation, J. Math. Phys., 40:12 (1999), 6473-6490.

[26] В. Э. Адлер, Классификация дискретных интегрируемых уравнений, Дисс. ... доктора физ.-матем. наук, Ин-т теорет. физики им. Л. Д. Ландау РАН, Черноголовка, 2010.

[27] J. L. Cieśliński, J. Phys. A, 42:40 (2009), 404003, 40 pp.

[28] S. Konstantinou-Rizos, A. V. Mikhailov, P. Xenitidis, "Reduction groups and related integrable difference systems of the NLS type", J. Math. Phys., 2013 (to appear).

[29] D. Zhang, D. Chen, J. Phys. A, 35:33 (2002), 7225-7241.

[30] D. K. Demskoi, V. V. Sokolov, Nonlinearity, 21:6 (2008), 1253-1264, arXiv: nlin/0607071.

[31] J. P. Wang, J. Math. Phys., 50:2 (2009), 023506, 25 pp., arXiv: 0809.3899.

[32] V.S. Gerdjikov, G. G. Grahovski, A. V. Mikhailov, T.I. Valchev, SIGMA, 7 (2011), 096, 48 pp., arXiv: 1108.3990 .

[33] A. S. Fokas, B. Fuchssteiner, Lett. Nuovo Cimento (2), 28:8 (1980), 299-303.

[34] P. J. Olver, J. Math. Phys., 18:6 (1977), 1212-1215.

[35] А.В. Михайлов, Дж.П. Ванг, П. Ксенитидис, ТМФ, 167:1 (2011), 23-49, arXiv: 1004.5346.

[36] B. A. Kupershmidt, Astérisque, 123 (1985), 21 pp.

[37] I. Dorfman, Dirac Structures and Integrability of Nonlinear Evolution Equations. Nonlinear Science: Theory and Applications, John Wiley and Sons, Chichester, 1993.

[38] A. De Sole, V. G. Kač, Non-local Poisson structures and applications to the theory of integrable systems, arXiv: 1302.0148.

[39] I. Cherdantsev, R. Yamilov, "Local master symmetries of differential-difference equations", Symmetries and Integrability of Difference Equations (Estérel, Québec, May 1994), CRM Proceedings and Lecture Notes, 9, eds. D. Levi, L. Vinet, P. Winternitz, AMS, Providence, RI, 1996, 51-61. 
[40] B. Fuchssteiner, W.-X. Ma, "An approach to master symmetries of lattice equations", Symmetries and Integrability of Difference Equations (Canterbury, 1996), London Mathematical Society Lecture Note Series, 255, eds. P. A. Clarkson, F. W. Nijhoff, Cambridge Univ. Press, Cambridge, 1999, 247-260.

[41] W. Oevel, H. Zhang, B. Fuchssteiner, Progr. Theor. Phys., 81:2 (1989), 294-308.

[42] I. Yu. Cherdantsev, R. I. Yamilov, Phys. D, 87:1-4 (1995), 140-144.

[43] R. I. Yamilov, J. Phys. A, 39:45 (2006), R541-R623.

[44] D. Levi, O. Ragnisco, Lett. Nuovo Cimento (2), 22:17 (1978), 691-696.

[45] O. I. Bogoyavlensky, Phys. Lett. A, 134:1 (1988), 34-38.

[46] С. В. Манаков, ЖЭТФ, 67:2 (1974), 543-555.

[47] R. Hirota, J. Phys. Soc. Japan, 35:1 (1973), 289-294.

[48] T. Tsuchida, M. Wadati, Chaos, Solitons \& Fractals, 9:6 (1998), 869-873.

[49] Р. И. Ямилов, УМН, 38:6(234) (1983), 153-160.

[50] A. Tongas, D. Tsoubelis, P. Xenitidis, J. Phys. A, 40:44 (2007), 13353-13384, arXiv: 0707.3730.

[51] P. Xenitidis, "Integrability and symmetries of difference equations: the Adler-Bobenko-Suris case", Proceedings of the 4th International Workshop in Group Analysis of Differential Equations and Integrable Systems (Protaras, Cyprus, 26-30 October 2008), 226-242, http://www2.ucy.ac.cy/ symmetry/Proceedings2008.pdf, arXiv: 0902.3954 .

[52] A. B. Shabat, R. I. Yamilov, Phys. Lett. A, 130:4-5 (1988), 271-275.

[53] A. V. Mikhailov, J. P. Wang, Phys. Lett. A, 375:45 (2011), 3960-3963, arXiv: 1105.1269.

[54] K. Narita, J. Math. Soc. Japan, 51:5 (1982), 1682-1685.

[55] Y. Itoh, Progr. Theor. Phys., 78:3 (1987), 507-510.

[56] A. K. Svinin, J. Phys. A, 42:45 (2009), 454021, 15 pp., arXiv: 0902.4517.

[57] A. K. Svinin, J. Phys. A, 44:16 (2011), 165206, 16 pp., arXiv: 1101.3808.

[58] V.É. Adler, On a discrete analog of the Tzitzeica equation, arXiv: 1103.5139.

[59] V.É. Adler, V. V. Postnikov, Differential-difference equations associated with the fractional Lax operators, arXiv: 1107.2305.

[60] M. Toda, J. Phys. Soc. Japan, 23:3 (1967), 501-506.

[61] H. Flaschka, Phys. Rev. B (3), 9:4 (1974), 1924-1925.

[62] R. Hirota, J. Phys. Soc. Japan, 35:1 (1973), 286-288.

[63] S. N. M. Ruijsenaars, Commun. Math. Phys., 133:2 (1990), 217-247.

[64] Y.B. Suris, J. Phys. A, 30:5 (1997), 1745-1761.

[65] W. Oevel, B. Fuchssteiner, H. Zhang, O. Ragnisco, J. Math. Phys., 30:11 (1989), 2664-2670.

[66] Yu. B. Suris, Rev. Math. Phys., 11:6 (1999), 727-822.

[67] Y. B. Suris, O. Ragnisco, Commun. Math. Phys., 200:2 (1999), 445-485.

[68] I. Merola, O. Ragnisco, G.-Z. Tu, Inverse Probl., 10:6 (1994), 1315-1334.

[69] V.É. Adler, R. I. Yamilov, J. Phys. A, 27:2 (1994), 477-492.

[70] M. J. Ablowitz, J. F. Ladik, J. Math. Phys., 17:6 (1976), 1011-1018.

[71] W. Hereman, J. A. Sanders, J. Sayers, J. P. Wang, "Symbolic computation of polynomial conserved densities, generalized symmetries, and recursion operators for nonlinear differential-difference equations", Group Theory and Numerical Analysis, CRM Proceedings and Lecture Notes, 39, eds. P. Winternitz, D. Gomez-Ullate, A. Iserles, D. Levi, P. J. Olver, R. Quispel, P. Tempesta, AMS, Providence, RI, 2005, 133-148.

[72] Ü. Göktaş, W. Hereman, Math. Computat. Appl., 16:1 (2011), 1-12.

[73] M. Bruschi, O. Ragnisco, Inverse Problems, 5:6 (1989), 983-998.

[74] T. Tsuchida, J. Phys. A, 35:36 (2002), 7827-7847, arXiv: nlin/0105053.

[75] В. Э. Адлер, ТМФ, 124:1 (2000), 48-61. 
[76] А. Б. Шабат, Р. И. Ямилов, Алгебра и анализ, 2:2 (1990), 183-208.

[77] A. A. Belov, K. D. Chaltikian, Phys. Lett. B, 317:1-2 (1993), 64-72, arXiv: hep-th/9305096.

[78] R. Sahadevan, S. Khousalya, J. Math. Anal. Appl., 280:2 (2003), 241-251.

[79] K. Hikami, R. Inoue, J. Phys. A, 30:19 (1997), 6911-6924.

[80] G. M. Beffa, J. P. Wang, Hamiltonian evolutions of twisted gons in $\mathbb{R P}^{n}$, arXiv: 1207.6524 .

[81] M. Blaszak, K. Marciniak, J. Math. Phys., 35:9 (1994), 4661-4682.

[82] V.É. Adler, A. I. Bobenko, Yu. B. Suris, Commun. Math. Phys., 233:3 (2003), 513-543, arXiv: nlin/0202024.

[83] В.Э. Адлер, А. И. Бобенко, Ю. Б. Сурис, Функи. анализ и его прил., 43:1 (2009), 3-21.

[84] D. Levi, R. I. Yamilov, J. Phys. A, 44:14 (2011), 145207, 22 pp., arXiv: 1011.0070. 